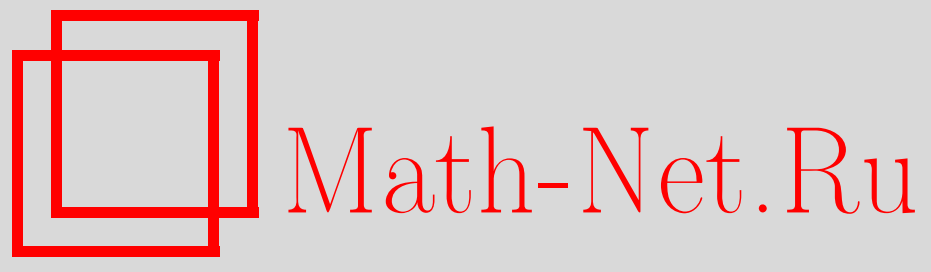

В. М. Бухштабер, Комплексные кобордизмы и формальные группы, УМH, 2012, том 67, выпуск 5, 111-174

DOI: https://doi.org/10.4213/rm9496

Использование Общероссийского математического портала Math-Net.Ru подразумевает, что вы прочитали и согласны с пользовательским соглашением http://www . mathnet.ru/rus/agreement

Параметры загрузки:

IP: 3.91 .87 .62

26 апреля 2023 г., $15: 28: 34$

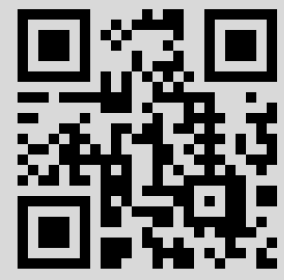




\section{Комплексные кобордизмы и формальные группы}

\section{В. М. Бухштабер}

Обзор посвящен современному состоянию теории кобордизмов. В центре внимания геометрические и универсальные свойства комплексных кобордизмов, алгебра Ландвебера-Новикова и формальная группа геометрических кобордизмов. Описываются связи с $K$-теорией, теорией алгебраических циклов, теорией формальных групп, теорией действия компактных групп Ли на многообразиях, торической топологией, теорией бесконечномерных алгебр Ли и теорией нильмногообразий. Обсуждаются ключевые результаты и нерешенные задачи.

Библиография: 124 названия.

Ключевые слова: алгебра Ландвебера-Новикова, операции АдамсаНовикова, характер Чженя-Дольда, спектральная последовательность Атья-Хирцебруха, род Хирцебруха.

\section{СОДЕРЖАНИЕ}

1. Введение.......................................... 112

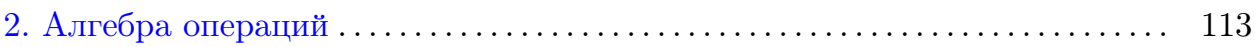

3. Спектральная последовательность Адамса-Новикова .............. 115

4. Классы Тома и гомоморфизмы Гизина ......................... 117

5. Мультипликативные преобразования........................ 123

6. Формальная группа геометрических кобордизмов ................. 125

7. Операции Адамса-Новикова и степенные системы.................. 128

8. Характеры Чженя и Чженя-Дольда .......................... 131

9. Спектральная последовательность Атья-Хирцебруха................. 137

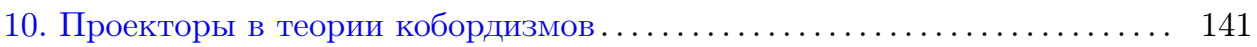

11. Двузначные формальные группы . . . . . . . . . . . . . . . . . . . 145

12. Квантовые группы и операторные дубли . . . . . . . . . . . . . . . . . 148

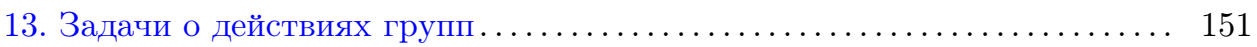

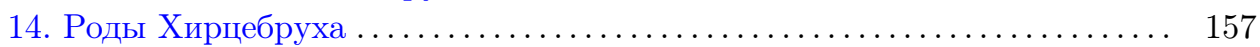

15. Эллиптические формальные группы . . . . . . . . . . . . . . . . . . . 160

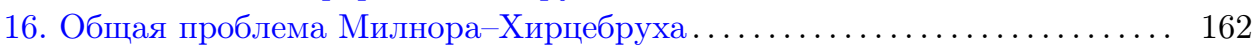

17. Алгебры Ли векторных полей и нильмногообразия. .............. 164

Работа выполнена при поддержке РФФИ (грант № 11-01-00694) и гранта Правительства РФ по постановлению № 220 (договор 11.G34.31.0053). 
18. Алгебраические кобордизмы ............................. 166

Список литературы . . . . . . . . . . . 167

\section{1. Введение}

Настоящий обзор представляет собой современную версию обзора [1].

Рекомендую прочесть энциклопедическую статью С. П. Новикова [2], а также его предисловие [3] к книге [4], содержащие оценки роли теории кобордизмов в современном развитии алгебраической топологии и ее связей с другими разделами математики. В [4] читатель найдет многие статьи, результаты которых обсуждаются в данном обзоре. Основные определения и конструкции можно найти в [5].

Понятие цикла в пространстве $X$ как образа отображения $f: M \rightarrow X$ гладкого замкнутого многообразия содержится в работе А. Пуанкаре [6]. Цикл считается гомологичным нулю, если отображение $f$ продолжается до отображения $W \rightarrow X$, где $W$ - гладкое многообразие с границей $\partial W=M$. Соответствующее понятие коцикла опирается на конструкцию Понтрягина-Тома, сопоставляющую вложению гладких многообразий $i: M_{1} \rightarrow M_{2}$, где $\partial M_{1}=\varnothing$ и $i\left(M_{1}\right) \cap \partial M_{2}=\varnothing$, отображение $M_{2} / \partial M_{2} \rightarrow T \nu$, где $\nu$ - нормальное расслоение вложения $i$ и $T \nu=D \nu / \partial D \nu$ - его пространство Тома, а $D \nu \subset \nu$ - подрасслоение со слоем единичный шар.

На основе этих понятий строятся теории гомологий и когомологий - теории бордизмов и кобордизмов в терминологии М. Атья (см. [7]).

Эти построения существенно опираются на фундаментальное понятие трансверсальной регулярности отображения многообразий [8].

Теории комплексных бордизмов $U_{*}(\cdot)$ и кобордизмов $U^{*}(\cdot)$ используют стабильно комплексные многообразия (U-многообразия), т. е. гладкие многообразия, допускающие вложения $M^{n} \subset \mathbb{R}^{N}$ с комплексным нормальным расслоением.

Группа $\Omega_{U}=U^{*}(\mathrm{pt}) \simeq U_{*}(\mathrm{pt})$ была независимо вычислена Дж. Милнором в [9] и С. П. Новиковым в [10]. Доказательство кольцевого изоморфизма $\Omega_{U} \simeq$ $\mathbb{Z}\left[a_{1}, \ldots, a_{n}, \ldots\right], \operatorname{deg} a_{n}=-2 n$, который известен как теорема Милнора-Новикова, впервые было опубликовано в [11].

В докладе С.П. Новикова на Международном математическом конгрессе (Москва, 1966) была выдвинута программа приложений теории комплексных кобордизмов к актуальным проблемам алгебраической топологии. Подробное изложение и развитие этой программы, использующее введенную А. С. Мищенко и С. П. Новиковым формальную группу геометрических кобордизмов, было дано в [12].

В предисловии к [12] говорится: "В процессе работы автору пришлось столкнуться с целым рядом новых и весьма заманчивых алгебраических и топологических ситуаций, аналоги которых в классическом случае либо полностью отсутствуют, либо сильно вырождаются; многие из них пока глубоко не рассмотрены. Все это позволяет высказать надежду на перспективность этого круга идей и методов как в отношении применений к известным классическим 
проблемам теории гомологий, так и в постановке и решении новых задач, от которых можно ждать появления нетрадиционных алгебраических связей и понятий".

Развитие алгебраических понятий, открытых в [12], можно найти в [13]-[17].

Обзор посвящен ключевым объектам программы С. П. Новикова - алгебре всех когомологических операций в теории комплексных кобордизмов и формальной группе геометрических кобордизмов.

В обзоре описано развитие постановок задач, понятий и связей, в которых алгебра всех когомологических операций и формальные группы в кобордизмах играют важную роль. В центре внимания - конструкции и результаты, актуальные:

- для алгебраической топологии, включая теорию родов Хирцебруха и теорию действия групп на многообразиях с приложениями алгебро-числовой теории формальных групп и теории эллиптических функций;

- для алгебры, включая теорию алгебр Хопфа, теорию квантовых групп (см. [18], [19]) и теорию операторных дублей как алгебр дифференциальных операторов (см. [16], [20]);

- для алгебраической геометрии, включая мотивную теорию (см. [21]) прямого образа и алгебраические кобордизмы с аналогом алгебры Ландвебера-Новикова (см. [15]).

\section{2. Алгебра операций}

Алгебра всех когомологических операций $\mathscr{A}_{U}$ в теории комплексных кобордизмов была описана С. П. Новиковым в [12]. В этой алгебре имеется подалгебра $S$, построенная на основе характеристических классов Чюеня-КоннераФлойда $c f_{k}(\xi), k=1,2, \ldots$, комплексных векторных расслоений $\xi$ (см. разд. 4 и 5). Алгебра $S$ имеет структуру связной градуированной алгебры Хопфа $S=\sum_{q \geqslant 0} S_{2 q}$, где $S_{0}=\mathbb{Z}$. Она была независимо введена С. П. Новиковым в [12] и П. Ландвебером в [22] и получила название алгебры Ландвебера-Новикова.

Единица 1 алгебры $S$ является образующей группы $S_{0}$. Базис группы $S_{2 q}$, $q>0$, образуют операции $s_{\omega}$, где $\omega$ пробегает все разбиения $\omega=\left(i_{1}, \ldots, i_{k}\right)$ числа $q$, т. е. $i_{l}>0$ и $\sum_{l=1}^{k} i_{l}=|\omega|=q$.

В аддитивном базисе $\left\{s_{\omega}\right\}$ диагональ в алгебре Хопфа $S$ задается формулой

$$
\Delta s_{\omega}=\sum_{\left(\omega^{\prime}, \omega^{\prime \prime}\right)=\omega} s_{\omega^{\prime}} \otimes s_{\omega^{\prime \prime}} .
$$

Следовательно, в каждой группе $S_{2 q}, q>0$, имеется в точности один примитивный элемент $s_{q}$, соответствующий разбиению $(q)$.

Имеет место изоморфизм $S_{*}=\operatorname{Hom}(S, \mathbb{Z})=\mathbb{Z}\left[m_{1}, \ldots, m_{n}, \ldots\right]$, где $m_{n}-$ элемент двойственный операции $s_{q}$ в аддитивном базисе $\left\{s_{\omega}\right\}$. Каноническое 
вложение $\Omega_{U} \subset S_{*}$ задается формулой $\left\langle\sigma, s_{\omega}\right\rangle=\varepsilon s_{\omega}(\sigma), \sigma \in \Omega_{U}$, где $\varepsilon: \Omega_{U} \rightarrow$ $\Omega_{U}^{0}=\mathbb{Z}$ аугментация кольца $\Omega_{U}$. Класс кобордизмов $\left[M^{2 n}\right]$ мультипликативно неразложим в кольце $\Omega_{U}$ тогда и только тогда, когда $s_{n}\left(\left[M^{2 n}\right]\right) \neq 0$.

Для любого клеточного пространства $X$ действие алгебры Хопфа $S$ на кольце $U^{*}(X)$ является милноровским (в терминологии работы [23]), т. е.

$$
s_{\omega}(a \cdot b)=\sum_{\left(\omega^{\prime}, \omega^{\prime \prime}\right)=\omega} s_{\omega^{\prime}}(a) s_{\omega^{\prime \prime}}(b) .
$$

В частности, операторы $s_{q}$ являются дифференцированиями колец $U^{*}(X)$.

Рассмотрим универсальное комплексное линейное расслоение $\eta \rightarrow \mathbb{C} P^{\infty}$, где $\mathbb{C} P^{\infty}$ - прямой предел комплексных проективных пространств $\mathbb{C} P^{n}$. Имеем $U^{*}\left(\mathbb{C} P^{\infty}\right)=\Omega_{U}[[u]]$, где $u=c f_{1}(\eta)$. Действие алгебры Хопфа $S$ на $U^{*}\left(\mathbb{C} P^{\infty}\right)$ задает ее милноровское действие на кольце $\mathbb{Z}[[u]]$ такое, что

$$
s_{\omega} u=\left\{\begin{array}{lll}
u^{q+1}, & \text { если } & \omega=(q) \\
0, & \text { если } & \omega \neq(q), q>0
\end{array}\right.
$$

Умножение в алгебре $S$ однозначно определяется тем, что ее соответствующее милноровское действие на $\mathbb{Z}\left[\left[u_{1}, \ldots, u_{N}\right]\right]$ индуцирует точное представление группы $\sum_{q=0}^{m} S_{2 q}$ для любого $m \leqslant N$. Из этого свойства и формулы (3) следует, что операция $s_{\omega}, \omega=\left(i_{1}, \ldots, i_{k}\right)$, определяется формулой

$$
s_{\omega} u(N)=u(N) c_{\omega},
$$

где $u(N)=u_{1} \cdots u_{N}, N \geqslant|\omega|$ и $c_{\omega}=c_{\omega}\left(u_{1}, \ldots, u_{N}\right)$ - симметрическая функция, соответствующая моному $u_{1}^{i_{1}} \cdots u_{k}^{i_{k}}$. Например,

$$
s_{q} u(N)=u(N) \sum u_{1}^{q} ; \quad s_{(1,1)} u(N)=u(N) \sum u_{1} u_{2} .
$$

Из формулы (4) выводятся формулы для композиции $s_{\omega_{1}} \cdot s_{\omega_{2}}$ операций $s_{\omega_{1}}$ и $s_{\omega_{2}}$, а также их коммутатора $\left[s_{\omega_{1}}, s_{\omega_{2}}\right]$. Например,

$$
s_{1}^{2}=2\left(s_{2}+s_{(1,1)}\right) .
$$

Важной является формула

$$
\left[s_{q_{1}}, s_{q_{2}}\right]=\left(q_{2}-q_{1}\right) s_{q_{1}+q_{2}}
$$

из которой, в частности, следует, что

$$
s_{2 q+1}=\left[s_{q}, s_{q+1}\right], \quad q \geqslant 1 ; \quad 2 s_{2 q}=\left[s_{q-1}, s_{q+1}\right], \quad q \geqslant 2 .
$$

Алгебра Хопфа, порожденная примитивными элементами, называется алгеброй Хопфа-Ли.

Ясно, что свободная градуированная алгебра $\mathscr{F}=\mathbb{Z}\left\langle s_{1}, \ldots, s_{n}, \ldots\right\rangle, \operatorname{deg} s_{n}=$ $2 n$, с диагональю $\Delta s_{n}=s_{n} \otimes 1+1 \otimes s_{n}$ является универсальной алгеброй 
Хопфа-Ли в категории алгебр Хопфа-Ли со счетным числом мультипликативных образующих.

Обозначим через $\widehat{S} \subset S$ образ канонического гомоморфизма алгебр Хопфа $\mathscr{F} \rightarrow S$. Имеем $S \otimes \mathbb{Q}=\widehat{S} \otimes \mathbb{Q}$ и $\widehat{S}\left[\frac{1}{2}\right] \simeq \mathbb{Z}\left[\frac{1}{2}\right]\left\langle s_{1}, s_{2}\right\rangle / J_{2}$, где $J_{2}$ - ядро гомоморфизма $\mathbb{Z}\left\langle s_{1}, s_{2}\right\rangle \rightarrow \widehat{S}$. Алгебра Хопфа $\widehat{S}$ определяет алгебру Ли с аддитивным базисом $s_{q}, q=1,2, \ldots$, и скобкой Ли (6).

Положим

$$
s_{1}(1)=\left[s_{1}, s_{2}\right] \quad \text { и } \quad s_{2}(1)=\left[s_{2}, s_{1}\right]=-s_{1}(1)
$$

и для $k>1$ введем индуктивно в свободной алгебре $\mathbb{Z}\left\langle s_{1}, s_{2}\right\rangle$ элементы

$$
s_{1}(k)=\left[s_{1}, s_{1}(k-1)\right] \quad \text { и } \quad s_{2}(k)=\left[s_{2}, s_{2}(k-1)\right] .
$$

Идеал $J_{2} \otimes \mathbb{Q}$ имеет две образующие

$$
s_{1}(3)+6 s_{2}(2) \quad \text { и } s_{1}(5)+40 s_{2}(3) .
$$

Алгебра $\mathscr{A}_{U}$ содержит также подкольцо $\Omega_{U}$ - алгебру операторов умножения на скаляры. Представление алгебры $S$ на кольце $\Omega_{U}$, соответствующее милноровскому действию когомологических операций на кольце когомологий точки, называется каноническим. В подходе С.П.Новикова большую роль играет точность этого представления, что принципиально отличает алгебру $S$ от классической алгебры Стинрода когомологических операций.

Операции из подалгебр $S$ и $\Omega_{U}$ алгебры $\mathscr{A}_{U}$ не коммутируют. Из $(2)$ вытекает правило коммутации

$$
s \sigma=\sum s^{\prime}(\sigma) s^{\prime \prime} .
$$

В [12] алгебра $\mathscr{A}_{U}$ описана как свободный левый $\Omega_{U}$-подмодуль с базисом $\left\{s_{\omega}\right\}$, т. е. $\mathscr{A}_{U} \cong \Omega_{U} S$ с правилом коммутации (8) операций $\sigma \in \Omega_{U}$ и $s \in S$.

Алгебра $\mathscr{A}_{U}$ обладает антиавтоморфизмом $\chi_{U}=\chi: \mathscr{A}_{U} \rightarrow \mathscr{A}_{U}$, т. е. $\chi(a \cdot b)=$ $\chi(b) \cdot \chi(a)$ для любых элементов $a, b$ из $A_{U}$, который однозначно задается свойствами: $\chi(\sigma)=\sigma, \sigma \in \Omega_{U}$, и $\chi\left(s_{q}\right)=-s_{q}$. Следовательно, для любых градуированных $\mathscr{A}_{U}$-модулей $M_{1}$ и $M_{2}$ определены градуированные $\mathbb{Z}$-модули $\operatorname{Ext}_{\mathscr{A}_{U}}^{*, *}\left(M_{1}, M_{2}\right)$ и $\operatorname{Tor}_{\mathscr{A}_{U}}^{*, *}\left(M_{1}, M_{2}\right)$.

В [12] показано, что $\operatorname{Ext}_{\mathscr{A}_{U}}^{0, *}\left(\Omega_{U}, \Omega_{U}\right)=\operatorname{Ext}_{\mathscr{A}_{U}^{0}}^{0,0}\left(\Omega_{U}, \Omega_{U}\right)=\mathbb{Z}$, и дано полное вычисление группы $\operatorname{Ext}_{\mathscr{A}_{U}}^{1, *}\left(\Omega_{U}, \Omega_{U}\right)$.

При помощи антиавтоморфизма $\chi$ из формулы (8) извлекается (см. [24]), что $\operatorname{Tor}_{\mathscr{A}_{U}}^{*, *}\left(\Omega_{U}, \Omega_{U}\right)=\Omega_{U} \otimes \mathscr{A}_{U} \Omega_{U}=0$.

\section{3. Спектральная последовательность Адамса-Новикова}

В работе [12] для широкого класса теорий когомологий и двойственных им теорий гомологий дана конструкция фильтраций и доказана сходимость соответствующих им спектральных последовательностей, которые получили название спектральных последовательностей Адамса-Новикова.

В случае, когда $X$ и $Y$ - конечные комплексы или прямые пределы конечных комплексов без кручения в обычных гомологиях, имеет место следующая теорема (см. [12]). 
Для теории комплексных кобордизмов $U^{*}(\cdot)$ существует функториальная по $X$ и $Y$ спектральная последовательность Адамса-Новикова $\left\{E_{r}^{s, *}, d_{r}, s \geqslant 0\right.$, $r \geqslant 2\}$, где $d_{r}: E_{r}^{s, t} \rightarrow E_{r}^{s+r, t+r-1} . \quad B$ этой спектральной последовательности $E_{2}^{s, t}=\operatorname{Ext}_{\mathscr{A} U}^{s, t}\left(U^{*}(Y), U^{*}(X)\right)$ и группь $\sum_{t-s=m} E_{\infty}^{s, t}$ связаны с группами стабильных гомотопических классов отображений с отмеченными точками $[X, Y]^{m}=\underset{n}{\lim }\left[\Sigma^{n+m} X, \Sigma^{n} Y\right]$ гомоморфизмами

$$
\begin{aligned}
& q_{0}^{m}:[X, Y]^{m} \rightarrow \operatorname{Hom}_{\mathscr{A} U}^{m}\left(U^{*}(Y), U^{*}(X)\right), \\
& q_{i}^{m}: \operatorname{ker} q_{i-1} \rightarrow E_{\infty}^{i, i+m}, \quad i>0 .
\end{aligned}
$$

Гомоморфизм

$$
q_{1}^{m}: \operatorname{ker} q_{0}^{m} \rightarrow \operatorname{Ext}_{\mathscr{A} U}^{1,1+m}
$$

называется $U$-инвариантом Хопфа.

Далее, говоря о спектральной последовательности Адамса-Новикова, мы будем иметь в виду описанную выше спектральную последовательность. Для $X=Y=S^{0}$ получается спектральная последовательность, сходящаяся к стабильным гомотопическим группам сфер $\pi_{*}^{s}$. В этом случае

$$
E_{2}^{*, *}=\operatorname{Ext}_{S}^{*, *}\left(\mathbb{Z}, \Omega_{U}\right)
$$

причем

$$
E_{2}^{0, *}=\operatorname{Hom}_{S}^{*}\left(\mathbb{Z}, \Omega_{U}\right)=\operatorname{Hom}_{S}^{0}\left(\mathbb{Z}, \Omega_{U}\right) \simeq \mathbb{Z}
$$

Таким образом,

$$
E_{\infty}^{2, *} \subset \operatorname{Ext}_{S}^{2, *}\left(\mathbb{Z}, \Omega_{U}\right)
$$

Следовательно, гомоморфизм $q_{2}^{m}$ задает гомоморфизм

$$
q_{2}^{m}: \operatorname{ker} q_{1}^{m} \rightarrow \operatorname{Ext}_{S}^{2,2+m}\left(\mathbb{Z}, \Omega_{U}\right) .
$$

В [12] показано, что группы $E_{r}^{1, t}, r \geqslant 2$, являются циклическими и $E_{4}^{1, t}=$ $E_{\infty}^{1, t}$. Образующие $\alpha_{t} \in E_{2}^{1, t}$ являются циклами всех дифференциалов при $t=2$, $t=4 s, t=4 s+1, s \geqslant 0$. Имеет место формула $d_{3} \alpha_{t}=h^{3} \alpha_{t-2}$ при $t=4 s-1$ и $4 s-2, s \geqslant 1$, где $h=\alpha_{1} \in E_{2}^{1,2}=\mathbb{Z} / 2$. Элементы $2 \alpha_{t}$ являются циклами всех дифференциалов. Образ $U$-инварианта Хопфа $q_{1}$ является эпиморфизмом при $t=4, t=8 s+1$ и $t=8 s+2, s \geqslant 1$, и подгруппа $\operatorname{Im} q_{1}$ имеет индекс 2 при $t=8 s+6, s \geqslant 0$, и $t=8 s+4, s \geqslant 1$. Для $i \leqslant 3$ определены гомоморфизмы

$$
q_{i}^{m}: \operatorname{ker} q_{i-1}^{m} \rightarrow \operatorname{Ext}_{S}^{i, i+m}\left(\mathbb{Z}, \Omega_{U}\right)
$$

где $\operatorname{ker} q_{i-1}^{m} \subset\left[S^{0}, S^{0}\right]^{m}=\pi_{m}^{s}$. Их называют инвариантами Новикова-Хопфа. Для вычисления этих инвариантов важно, что они согласованы с умножением в кольце $\operatorname{Ext}_{S}\left(\mathbb{Z}, \Omega_{U}\right)$ и умножением Масси в нем. Отсюда сразу вытекает, что если $q_{1}^{m}(\alpha) \neq 0$, где $\alpha \in \pi_{m}^{s}$, то $\alpha$ мультипликативно неразложим в кольце $\pi_{*}^{s}$.

В [25] при помощи инварианта $q_{2}$ построено семейство нетривиальных произведений Масси в кольце $\pi_{*}^{s}$. 
Спектральная последовательность Адамса-Новикова является основным инструментом для изучения стабильных гомотопических групп сфер (см. [26]). Среди важнейших достижений в этом направлении отметим результат по известной проблеме арф-инварианта Кервера

$$
k: \pi_{4 n+2}^{s} \rightarrow \mathbb{Z} / 2 \subset E_{2}^{2,4(n+1)} .
$$

Согласно теореме Ревенеля-Хилла-Хопкинса (2009 г.) этот инвариант тривиален во всех размерностях, за исключением только $n=0,1,3,7,15$ и, возможно, 31 (см. обзор [27]).

\section{4. Классы Тома и гомоморфизмы Гизина}

Пусть $h^{*}(\cdot)$ - некоторая мультипликативная теория когомологий. Обозначим через $\Omega_{h}=\sum \Omega_{h}^{i}$ кольцо скаляров $h^{*}(\mathrm{pt})$. Будем считать, что $\Omega_{h}$ - кольцо с единицей $1 \in \Omega_{h}^{0}$.

Рассмотрим векторное $n$-мерное расслоение $\zeta \rightarrow X$ над связным клеточным комплексом $X$. Вложение слоя $i_{x}: \mathbb{R}^{n} \rightarrow \zeta$ над точкой $x \in X$ задает отображение пространств Тома $T_{i_{x}}: S^{n}=T \mathbb{R}^{n} \rightarrow T \zeta$.

Расслоение $\zeta$ называется ориентируемым в теории $h^{*}(\cdot)$ ( $h$-ориентируемым), если гомоморфизм $\left(T_{i_{x}}\right)^{*}: \widetilde{h}^{n}(T \zeta) \rightarrow \widetilde{h}^{n}\left(S^{n}\right)$ является эпиморфизмом. Элемент $a \in \widetilde{h}^{n}(T \zeta)$ такой, что $\left(T_{i_{x}}\right)^{*} a=\Sigma^{n} \cdot 1$, называется ориентацией расслоения $\zeta$ в теории $h^{*}(\cdot)$. Здесь $\Sigma^{n}: \Omega_{h}^{0} \rightarrow \widetilde{h}^{n}\left(S^{n}\right)-$ изоморфизм надстройки. Ориентация $a$ расслоения $\zeta$ называется также классом Тома этого расслоения в теории $h^{*}(\cdot)$ и обозначается $u_{h}(\zeta)$ (объяснение см. в этом разделе ниже).

Например, любое векторное расслоение ориентируемо в обычных когомологиях $H^{*}(\cdot ; \mathbb{Z} / 2)$ и имеет единственную ориентацию, так как $H^{*}(\mathrm{pt} ; \mathbb{Z} / 2)=$ $H^{0}(\mathrm{pt} ; \mathbb{Z} / 2)=\mathbb{Z} / 2$. Любое векторное расслоение ориентируемо и в теории неориентированных кобордизмов $O^{*}(\cdot)$, но уже может иметь много ориентаций, так как $O^{*}(\mathrm{pt})=\Omega_{O}$ имеет нетривиальные элементы отрицательной размерности. Векторное расслоение является ориентируемым в классическом смысле тогда и только тогда, когда его первый класс Штифеля-Уитни $w_{1}(\zeta)$ равен нулю. Такие расслоения ориентируемы в классических когомологиях $H^{*}(\cdot, \mathbb{Z})$ и имеют только две ориентации, так как $H^{*}(\mathrm{pt} ; \mathbb{Z})=H^{0}(\mathrm{pt}, \mathbb{Z})=\mathbb{Z}$. Они ориентируемы в теории ориентированных кобордизмов $S O^{*}(\cdot)$ и имеют много ориентаций, так как кольцо $S O^{*}(\mathrm{pt})=\Omega_{S O}$ имеет нетривиальные элементы отрицательной размерности.

Существует понятие $h^{*}$-ориентации, универсальной для определенного класса расслоений. Опишем необходимую нам $U^{*}$-ориентацию, универсальную для комплексных расслоений.

Теория комплексных кобордизмов $U^{*}(\cdot)$ задается спектром Тома

$$
M U=\left\{T_{n} ; \varepsilon_{n}: \Sigma T_{n} \rightarrow T_{n+1}, n \geqslant 0\right\}, \quad \text { т. е. } \quad U^{k}(X)=\underset{n}{\lim }\left[\Sigma^{n} X, T_{n+k}\right],
$$

где $T_{2 n}=T \xi_{n}=M U(n)$ - пространство Тома универсального $n$-мерного комплексного расслоения $\xi_{n} \rightarrow B U(n)$ и $B U(n)$ - прямой предел многообразий 
Грассмана $\mathbb{C} G_{N, n}$ комплексных $n$-мерных подпространств в $\mathbb{C}^{N}$. По определению, $T_{2 n+1}=\Sigma T_{2 n}$ и $\varepsilon_{2 n}$ - тождественное отображение, а $\varepsilon_{2 n+1}: \Sigma^{2} T_{2 n} \rightarrow$ $T_{2(n+1)}$ соответствует отображению

$$
\Sigma^{2} M U(n)=T\left(\xi_{n} \oplus 1\right) \rightarrow T\left(\xi_{n+1}\right)=M U(n+1),
$$

где 1 - одномерное комплексное тривиальное расслоение. Отметим, что $B U(0)=$ pt, $\xi_{0}=$ pt, $T_{0}=M U(0)=S^{0}-$ нульмерная сфера, $T_{2}=M U(1)=\mathbb{C} P^{\infty}$ и $\varepsilon_{2}: S^{2} \rightarrow \mathbb{C} P^{\infty}-$ стандартное вложение.

Имеем $\Omega_{U}=\pi_{*}(M U)$, единица $1 \in \Omega_{U}$ соответствует тождественному отображению $T_{0} \rightarrow T_{0}$ и порождает каноническое отображение спектров $i_{a}^{U}: S \rightarrow M U$, где $S=\left\{S^{n}, n \geqslant 0\right\}$ - спектр сфер, $i^{U}=\left\{i_{n}^{U}=\Sigma^{n} \varepsilon_{0}: S^{n} \rightarrow T_{n}\right\}$ и $i_{2}^{U}=\varepsilon_{2}$.

Каждое комплексное $n$-мерное расслоение $\zeta \rightarrow X$ над клеточным пространством классифицируется отображением $f_{\zeta}: X \rightarrow B U(n)$, которое задает отображение пространств Тома $T_{f_{\zeta}}: T \zeta \rightarrow M U(n)$ и, следовательно, класс кобордизмов $u(\zeta) \in U^{2 n}(T \zeta)$. По построению, класс $u(\zeta)$ является $U^{*}$-ориентацией (классом Тома) расслоения $\zeta$.

Совершенно аналогично строятся $h^{*}$-ориентации, универсальные для всех векторных расслоений, когда $h^{*}(\cdot)=O^{*}(\cdot)$, ориентированных векторных расслоений, когда $h^{*}(\cdot)=S O^{*}(\cdot)$, комплексных векторных расслоений с нулевым первым классом Чженя, когда $h^{*}(\cdot)=S U^{*}(\cdot)$, комплексных расслоений с кватернионной структурой, когда $h^{*}(\cdot)=S p^{*}(\cdot)$, где $O^{*}(\cdot), S O^{*}(\cdot), S U^{*}(\cdot)$, $S p^{*}(\cdot)$ - теории кобордизмов, задаваемые спектрами Тома $M O=\{M O(n)\}$, $M S O=\{M S O(n)\}, M S U=\{M S U(n)\}$ и $M S p=\{M S p(n)\}$ соответственно (cм. [11], [28]).

В теориях $O^{*}(\cdot)$ и $S p^{*}(\cdot)$ на основе универсальных ориентаций и соответствующих характеристических классов так же, как в теории комплексных кобордизмов, строятся аналоги алгебры операций Ландвебера-Новикова.

Вернемся к общему случаю.

Классы Тома обладают функториальными свойствами:

1. Пусть $f: X_{1} \rightarrow X_{2}$ - непрерывное отображение и $\zeta \rightarrow X_{2}$ - расслоение с классом Тома $u_{h}(\zeta)$. Тогда класс $f^{*}\left(u_{h}(\zeta)\right)$ является классом Тома индуцированного расслоения $f^{*} \zeta$, т. е. $f^{*}\left(u_{h}(\zeta)\right)=u_{h}\left(f^{*} \zeta\right)$.

2. Пусть $\mu: h_{1}^{*}(\cdot) \rightarrow h_{2}^{*}(\cdot)$ - преобразование теорий когомологий и $u_{h_{1}}(\zeta)-$ класс Тома расслоения $\zeta \rightarrow X$. Тогда класс $\mu u_{h_{1}}(\zeta)$ является классом Тома в теории $h_{2}$, т. е. $\mu u_{h_{1}}(\zeta)=u_{h_{2}}(\zeta)$.

Далее, если будет понятно, о какой теории $h^{*}(\cdot)$ идет речь, мы будем писать $u$ вместо $u_{h}$.

Пусть $\zeta_{k} \rightarrow X_{k}, k=1,2,-$ векторные расслоения. Для расслоения $\zeta_{1} \times \zeta_{2} \rightarrow$ $X_{1} \times X_{2}$ имеет место гомеоморфизм

$$
T\left(\zeta_{1} \times \zeta_{2}\right) \cong T\left(\zeta_{1}\right) \wedge T\left(\zeta_{2}\right) .
$$

Тогда, если $u_{k}, k=1,2,-h$-ориентации расслоений $\zeta_{k}$, то класс $u_{1} \otimes u_{2}$ является $h$-ориентацией расслоения $\zeta_{1} \times \zeta_{2}$. В случае $X_{1}=X_{2}=X$ при помощи диагонального отображения $\Delta: X \rightarrow X \times X$ мы получаем $h$-ориентацию суммы Уитни $\zeta_{1} \oplus \zeta_{2} \rightarrow X$ расслоений $\zeta_{1}$ и $\zeta_{2}$. 
В зависимости от задачи возникают разные конструкции классов Тома $u_{h}(\zeta)$ расслоений. Конструкция называется мультипликативной, если $u_{h}\left(\zeta_{1} \times \zeta_{2}\right)=$ $u_{h}\left(\zeta_{1}\right) \otimes u_{h}\left(\zeta_{2}\right)$. Теория комплексных кобордизмов $U^{*}(\cdot)$ является мультипликативной. Умножение задается отображением спектров Тома $M U \wedge M U \rightarrow M U$ при помощи отображений пространств Тома $M U\left(n_{1}\right) \wedge M U\left(n_{2}\right) \rightarrow M U\left(n_{1}+n_{2}\right)$, соответствующих отображениям $B U\left(n_{1}\right) \times B U\left(n_{2}\right) \rightarrow B U\left(n_{1}+n_{2}\right)$, классифицирующим расслоения $\xi_{n_{1}} \times \xi_{n_{2}} \rightarrow B U\left(n_{1}\right) \times B U\left(n_{2}\right)$. Приведенная выше конструкция $U^{*}$-ориентации, универсальной для комплексных векторных расслоений, является мультипликативной, что непосредственно вытекает из конструкции умножения в теории комплексных кобордизмов.

Имеем $T \zeta=D \zeta / \partial D \zeta$. Пространство единичных шаров $D \zeta$ гомотопически эквивалентно пространству $X$, поэтому диагональное отображение $\Delta: D \zeta \rightarrow$ $D \zeta \times D \zeta$ задает отображение $\Delta_{T}: X \rightarrow X \wedge T \zeta$ и, следовательно, индуцирует гомоморфизм $\Delta_{T}^{*}: h^{*}(X) \otimes h^{*}(T \zeta) \rightarrow h^{*}(T \zeta)$.

Для любого класса Тома $u(\zeta)$ векторного расслоения $\zeta \rightarrow X$ над клеточным пространством $X$ гомоморфизм $\Delta_{T}^{*}$ задает изоморфизм Тома:

$$
T_{u(\zeta)}: h^{k}(X) \rightarrow \widetilde{h}^{k+n}(T \zeta): T_{u(\zeta)} x=u(\zeta) x,
$$

где $n=\operatorname{dim}_{\mathbb{R}} \zeta$ и $x \in h^{k}(X)$.

Как и в случае классических когомологий, ориентация $u(\zeta)$ задает эйлеров класс $\chi(\zeta) \in \widetilde{h}^{n}(X)$ по формуле $\chi(\zeta)=i^{*} u(\zeta)$, где $i: X \rightarrow T \zeta$ - вложение, определяемое нулевым сечением расслоения $\zeta$. Если расслоение $\zeta \rightarrow X$ обладает сечением $s: X \rightarrow \zeta$ таким, что $\|s(x)\| \neq 0$ для всех $x \in X$, то $\chi(\zeta)=0$.

Пусть $u_{1}(\zeta)$ и $u_{2}(\zeta)$ - две $h$-ориентации $n$-мерного расслоения $\zeta \rightarrow X$. Тогда определен класс $\rho\left(u_{1}(\zeta), u_{2}(\zeta)\right) \in h^{0}(X)$ такой, что

$$
T_{u_{2}(\zeta)} \rho\left(u_{1}(\zeta), u_{2}(\zeta)\right)=u_{1}(\zeta)
$$

различающий эти ориентации. Из функториальности изоморфизма Тома следует, что этот класс функториален, т. е.

$$
f^{*} \rho\left(u_{1}(\zeta), u_{2}(\zeta)\right)=\rho\left(u_{1}\left(f^{*} \zeta\right), u_{2}\left(f^{*} \zeta\right)\right)
$$

где $f: X_{1} \rightarrow X_{2}$ - непрерывное отображение и $\zeta \rightarrow X_{2}$ - расслоение над $X_{2}$. Напомним конструкцию характеристических классов расслоений как различающих классов специально подобранных универсальных ориентаций.

Пусть $h_{1}(\cdot)$ и $h_{2}(\cdot)$ - мультипликативные теории когомологий, обладающие универсальными ориентациями $u_{1}(\zeta)$ и $u_{2}(\zeta)$ для данного класса расслоений. Далее без ограничения общности мы будем рассматривать класс комплексных расслоений.

Возьмем некоторое мультипликативное преобразование $\mu: h_{1}(\cdot) \rightarrow h_{2}(\cdot)$ и сопоставим комплексному расслоению $\zeta \rightarrow X$ класс $\chi_{\mu}(\zeta)=\chi_{\mu}\left(\zeta ; h_{1}, h_{2}\right) \in$ $h_{2}^{0}(X)$ по формуле

$$
u_{2}(\zeta) \chi_{\mu}(\zeta)=\mu u_{1}(\zeta)
$$

т. е. $\chi_{\mu}(\zeta)=\rho\left(\mu u_{1}(\zeta), u_{2}(\zeta)\right)$. 
Непосредственно из конструкции следует, что мы получаем характеристический класс комплексных расслоений такой, что $\chi_{\mu}(1)=1$ и $\chi_{\mu}\left(\zeta_{1} \oplus \zeta_{2}\right)=$ $\chi_{\mu}\left(\zeta_{1}\right) \chi_{\mu}\left(\zeta_{2}\right)$. Из универсальности ориентаций $u_{1}(\zeta)$ и $u_{2}(\zeta)$ следует, что этот характеристический класс однозначно определяется условием

$$
u_{2}(\eta) \chi_{\mu}(\eta)=\mu u_{1}(\eta)
$$

где $\eta \rightarrow \mathbb{C} P^{\infty}$ - универсальное одномерное комплексное расслоение.

Пример. Пусть $h_{1}^{*}(\cdot)=U^{*}(\cdot)$ и $h_{2}^{*}(\cdot)=U^{*}(\cdot)[[\beta]]$, где $\operatorname{deg} \beta=-2$. Возьмем в качестве $u_{1}$ описанную выше универсальную $U^{*}$-ориентацию и в качестве $u_{2}$ ее же для теории $U^{*}(\cdot)[[\beta]]$. Тогда для мультипликативного преобразования

$$
\mu: U^{*}(\cdot) \rightarrow U^{*}(\cdot)[[\beta]]
$$

такого, что $\mu u_{1}(\eta)=u_{1}(\eta)+u_{1}(\eta)^{2} \beta$, мы получаем

$$
\chi_{\mu}(\eta)=1+u_{1}(\eta) \beta,
$$

и, следовательно, для любого комплексного $n$-мерного расслоения $\zeta \rightarrow X$ мы получаем

$$
\chi_{\mu}(\zeta)=1+c_{1}(\zeta) \beta+\cdots+c_{n}(\zeta) \beta^{n},
$$

где $c_{k}(\zeta)=c f_{k}(\zeta), k=1, \ldots, n,-$ характеристические классы Чженя-КоннераФлойда.

Говоря о многообразиях и их отображениях, мы предполагаем, что они обладают необходимой гладкостью. Рассмотрим замкнутое многообразие $M^{m}$ и многообразие $W^{l}$, быть может с непустой границей $\partial W^{l}$. Будем рассматривать отображения $f: M^{m} \rightarrow W^{l}$ с условием $f(M) \cap \partial W=\varnothing$. Такое отображение называется $h$-ориентируемым, если существует вложение $j: M^{m} \rightarrow \mathbb{R}^{q+m}$ такое, что $n$-мерное нормальное расслоение $\zeta \rightarrow M, n=q+l$ вложения $f \times j: M^{m} \rightarrow$ $W^{l} \times \mathbb{R}^{q+m}$ является $h$-ориентируемым. Имеем отображение Понтрягина-Тома $P T: \Sigma^{q+m}(W / \partial W) \rightarrow T \zeta$.

Для любого $h$-ориентированного отображения $f: M \rightarrow W$ с $h$-ориентацией $u(\zeta)$ определен гомоморфизм Гизина (прямой образ) в теории $h^{*}(\cdot)$

$$
f_{!}=\left(f_{u(\zeta)}\right) !: h^{k}\left(M^{m}\right) \rightarrow h^{k+l-m}\left(W^{l}, \partial W^{l}\right)
$$

по формуле

$$
\Sigma^{q+m} f_{!} x=(P T)^{*}(u(\zeta) x) .
$$

Здесь $\Sigma$ - изоморфизм надстройки.

По построению, гомоморфизм Гизина зависит от выбора $h$-ориентации $u(\zeta)$ нормального расслоения $\zeta \rightarrow M$ вложения $M^{m} \rightarrow W^{l} \times \mathbb{R}^{q+m}$.

Отображение пар $f:(M, \varnothing) \rightarrow(W, \partial W)$ индуцирует кольцевой гомоморфизм $f^{*}: h^{*}(W, \partial W) \rightarrow h^{*}(M)$ и, следовательно, структуру $h^{*}(W, \partial W)$-модуля в кольце $h^{*}(M)$. Имеет место формула:

$$
f_{!}\left(x \cdot f^{*}(y)\right)=f_{!}(x) \cdot y, \quad x \in h^{*}(M), \quad y \in h^{*}(W, \partial W),
$$

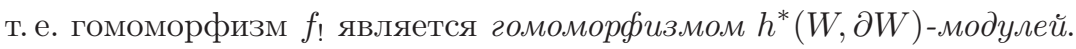


Из формулы для $f$ ! выводится правило коммутации стабильных когомологических операций с гомоморфизмом Гизина в теории $h^{*}(\cdot)$. Например, для операций $s_{n}$ и универсального класса Тома $u(\zeta)$ в $U^{*}$-теории (см. выше) имеет место формула

$$
s_{n} f_{!} x=f_{!} s_{n} x+f_{!}\left(c f_{(n)}(\zeta) x\right),
$$

где $c f_{(n)}(\zeta)$ - характеристический класс, однозначно определяемый тем, что $c f_{(n)}\left(\zeta_{1} \oplus \zeta_{2}\right)=c f_{(n)}\left(\zeta_{1}\right)+c f_{(n)}\left(\zeta_{2}\right)$ и $c f_{(n)}(\zeta)=u(\zeta)^{n}$, если $\operatorname{dim}_{\mathbb{C}} \zeta=1$.

Пусть $u_{1}$ и $u_{2}$ - две $h$-ориентации расслоения $\zeta$. Непосредственно из конструкции гомоморфизма Гизина и класса $\rho\left(u_{1}(\zeta), u_{2}(\zeta)\right)=\rho\left(u_{1}, u_{2}\right)$ следует формула

$$
f_{u_{1}, !} x=f_{u_{2}, !}\left(\rho\left(u_{1}, u_{2}\right) x\right), \quad x \in h^{k}(M) .
$$

Пусть теперь даны мультипликативные теории когомологий $h_{1}(\cdot)$ и $h_{2}(\cdot)$ и мультипликативное преобразование $\mu: h_{1}(\cdot) \rightarrow h_{2}(\cdot)$. Тогда для любого отображения $f: M \rightarrow W$ с нормальным расслоением $\varsigma$ такого, что существуют ориентации $u_{1}(\zeta) \in h_{1}^{l+q}(T \zeta)$ и $u_{2}(\zeta) \in h_{2}^{l+q}(T \zeta)$, имеет место формула

$$
\mu f_{!, h_{1}} x=f_{!, h_{2}}\left(\rho\left(\mu u_{h_{1}}, u_{h_{2}}\right) x\right) .
$$

Ясно, что формула (13) является частным случаем формулы (14), когда $h_{1}=h_{2}=h$ и $\mu$ - тождественное преобразование.

Многообразие $M^{m}$ называется $h$-ориентируемым, если проекция $\pi: M^{m} \rightarrow \mathrm{pt}$ является $h$-ориентируемым отображением. $h$-ориентированным многообразием называется пара $\left(M^{m}, u(\nu)\right)$, где $M^{m}-h$-ориентируемое многообразие и $u(\nu)$ - фиксированная $h$-ориентация нормального расслоения $\nu$ вложения $j: M^{m} \rightarrow \mathbb{R}^{n+m}$.

Пусть $\left(M_{1}, u\left(\nu_{1}\right)\right)$ и $\left(M_{2}, u\left(\nu_{2}\right)\right)$ - два замкнутых $h$-ориентированных многообразия, где $\nu_{k}$ - нормальное расслоение вложения $M_{k}=M_{k}^{m_{k}} \subset \mathbb{R}^{m_{k}+n_{k}}$, $k=1,2$.

Тогда любое гладкое отображение $f: M_{1} \rightarrow M_{2}$ является $h$-ориентируемым с $h$-ориентацией $u(\zeta)$, которая определяется $h$-ориентациями $u\left(\nu_{1}\right)$ и $u\left(\nu_{2}\right)$ при помощи соотношения

$$
\tau_{1} \oplus \zeta=f^{*} \tau_{2}+\left[m_{2}+n_{1}\right]
$$

где $\zeta$, как и выше, нормальное расслоение вложения $M_{1} \subset M_{2} \times \mathbb{R}^{m_{1}+n_{1}}$ и $\tau_{k}-$ касательное расслоение многообразия $M_{k}, k=1,2$.

Пусть дано мультипликативное преобразование $\mu: h_{1}(\cdot) \rightarrow h_{2}(\cdot)$.

Сопоставим каждому многообразию $M$ с ориентациями $u_{k}(\nu)$ в теориях $h_{k}(\cdot)$ класс $\chi_{\mu}(M)$ такой, что

$$
u_{2}(\tau(M)) \chi_{\mu}(M)=\mu u_{1}(\tau(M)) .
$$

Рассмотрим теперь гладкое отображение $f: M_{1} \rightarrow M_{2}$ многообразий, каждое из которых имеет ориентации в мультипликативных теориях $h_{1}(\cdot)$ и $h_{2}(\cdot)$, тогда для любого мультипликативного преобразования $\mu: h_{1}(\cdot) \rightarrow h_{2}(\cdot)$ имеет место формула

$$
\mu\left(f_{!, 1} x\right) \chi_{\mu}\left(M_{2}\right)=f_{!, 2}\left(\mu(x) \chi_{\mu}\left(M_{1}\right)\right),
$$

где $f_{!, k}: h_{k}^{*}\left(M_{1}\right) \rightarrow h_{k}^{*}\left(M_{2}\right)$ - гомоморфизм Гизина в теории $h_{k}^{*}(\cdot), k=1,2$. 
В случае, когда $h_{1}(\cdot)$ - комплексная $K$-теория, $h_{2}(\cdot)$ - обычные когомологии $H^{*}(\cdot, \mathbb{Q})$ и $\mu=\mathrm{ch}: K^{*}(\cdot) \rightarrow H^{*}(\cdot, \mathbb{Q})$ - классический характер Чженя, формула (15) является теоремой Римана-Роха-Хирцебруха-Гротендика [29], [30], где $\chi(M)=\operatorname{Td}(M)$ - род Тодда многообразия $M$. Это топологический аналог знаменитого результата алгебраической геометрии. Напомним, что для алгебраического $n$-мерного многообразия $M$ имеет место формула

$$
\operatorname{Td}(M)=\sum_{i=0}^{n}(-1)^{i} g_{i},
$$

где $g_{i}$ - комплексная размерность пространства голоморфных дифференциальных $i$-мерных форм на $M$. Общий случай изложен в [31].

Пусть $M_{1}$ и $M_{2}$ - замкнутые многообразия, $f_{1}: M_{1} \rightarrow M_{2}$ и $f_{2}: M_{2} \rightarrow W-$ отображения с $h$-ориентациями $u_{1}$ и $u_{2}$. Тогда композиция отображений

$$
f_{2} f_{1}: M_{1} \rightarrow M_{2} \rightarrow W
$$

является $h$-ориентируемой. Более того, можно выбрать $h$-ориентацию отображения $f_{2} f_{1}$ так, что

$$
\left(f_{2} f_{1}\right) !=f_{2, !} f_{1, !} .
$$

В контексте алгебраической геометрии над произвольными полями теория классов Тома и гомоморфизмов Гизина была систематически развита в работах [32]-[34].

Вложение точки $\mathrm{pt} \rightarrow W^{l}$ является $h$-ориентируемым отображением для любой теории $h^{*}(\cdot)$. Прямой образ в этом случае представляет собой гомоморфизм

$$
\Omega_{h}^{k}=h^{k}(\mathrm{pt}) \rightarrow h^{k+l}\left(W^{l}, \partial W^{l}\right),
$$

индуцированный канонической проекцией $W^{l} / \partial W^{l} \rightarrow S^{l}$.

Для $h$-ориентированного многообразия $\left(M^{m}, u(\nu)\right)$ прямой образ для проекции $\pi: M^{m} \rightarrow p t$ представляет собой гомоморфизм

$$
h^{k}\left(M^{m}\right) \rightarrow h^{k-m}(\mathrm{pt})=\Omega_{h}^{k-m} .
$$

Говорят, что многообразие $M^{m}$ обладает стабильно комплексной структурой, если существует вложение $j: M^{m} \rightarrow \mathbb{R}^{m+2 n}$ такое, что $2 n$-мерное нормальное расслоение $\nu$ допускает структуру $n$-мерного комплексного расслоения. Такое многообразие вместе с выбранной структурой комплексного расслоения для $\nu$ называется стабильно комплексным. Для описанной выше универсальной $U^{*}$-ориентации $u(\zeta)$ имеет место формула

$$
\pi ! 1=\left[M^{m}\right] \in \Omega_{U}^{-m} .
$$

Рассмотрим композицию

$$
\mathrm{pt} \stackrel{i}{\rightarrow} M^{m} \stackrel{\pi}{\rightarrow} \mathrm{pt}
$$


где $M^{m}$ - замкнутое $h$-ориентированное многообразие. В этом случае тождественное отображение $\Omega_{h}^{0} \rightarrow \Omega_{h}^{0}$ разлагается в композицию

$$
\Omega_{h}^{0} \stackrel{i_{!}}{\rightarrow} h^{m}\left(M^{m}\right) \stackrel{\pi_{!}}{\longrightarrow} \Omega_{h}^{0} .
$$

Таким образом, для каждого $h$-ориентированного многообразия $\left(M^{m}, u(\zeta)\right)$ в группе $h^{m}\left(M^{m}\right)$ имеется прямое слагаемое $\Omega_{h}^{0}$, порожденное элементом $\left\langle M^{m}\right\rangle=i_{1} 1$, который называется его $h$-фундаментальным классом. Ясно, что этот класс определяется $h$-ориентацией $u(\zeta)$.

Для гладкого многообразия $W^{l}$ с границей $\partial W^{l}$ группа комплексных кобордизмов $U^{k}\left(W^{l}, \partial W^{l}\right)$ определяется геометрически (см. детали в [35]) как группа классов эквивалентности $U$-ориентированных отображений

$$
f: M^{l-k} \rightarrow W, \quad \partial M^{l-k}=\varnothing, \quad f\left(M^{l-k}\right) \cap \partial W=\varnothing .
$$

Пусть теперь $W$ обладает вложением $i_{W}: W \subset \mathbb{R}^{N_{1}}$ с комплексным нормальным расслоением $\nu_{W}$, т. е. $W$ является $U$-многообразием. Тогда любому $U$-ориентированному отображению $f: M^{l-k} \rightarrow W$, т. е. отображению с комплексным нормальным расслоением $\nu_{f}$ вложения $f \times i_{M}: M^{l-k} \subset W \times \mathbb{R}^{N_{2}}$ отвечает комплексное нормальное расслоение вложения

$$
\left(i_{W} \times 1\right)\left(f \times i_{M}\right): M^{l-k} \subset \mathbb{R}^{N_{1}} \times \mathbb{R}^{N_{2}},
$$

т. е. структура $U$-многообразия на $M^{l-k}$.

В этом случае определен геометрический оператор двойственности Пуанкаре в комплексных кобордизмах, который сопоставляет $k$-мерному $U$-коциклу $[f] \in U^{k}\left(W^{l}, \partial W^{l}\right)$ класс эквивалентности отображения $f: M^{l-k} \rightarrow W$ относительно индуцированной $U$-структуры на $M^{l-k}$, т. е. $(l-k)$-мерный цикл $\mathscr{D}[f] \in U_{l-k}\left(W^{l}\right)$.

Описанный оператор $\mathscr{D}$ задает изоморфизм групп

$$
\mathscr{D}: U^{k}\left(W^{l}, \partial W^{l}\right) \rightarrow U_{l-k}\left(W^{l}\right)
$$

и изоморфизм $\Omega_{U}$-модулей

$$
\mathscr{D}: U^{*}\left(W^{l}, \partial W^{l}\right) \rightarrow U_{l-*}\left(W^{l}\right) .
$$

В случае $W^{l}=$ pt это - изоморфизм

$$
\mathscr{D}: \Omega_{U}^{-2 n}=U^{-2 n}(\mathrm{pt}) \rightarrow U_{2 n}(\mathrm{pt})=\Omega_{2 n}^{U} .
$$

\section{5. Мультипликативные преобразования}

Теория когомологий $h^{*}(\cdot)$ называется $\mathbb{C}$-ориентируемой, если универсальное комплексное линейное расслоение $\xi \rightarrow \mathbb{C} P^{\infty}$ является ориентируемым в теории $h^{*}(\cdot)$. Используя гомотопическую эквивалентность $\mathbb{C} P^{\infty} \cong T \xi$, можно дать следующее определение.

$\mathbb{C}$-ориентированной теорией называется пара $\left(h^{*}(\cdot), u_{h}\right)$, где $h^{*}(\cdot)$ - некоторая $\mathbb{C}$-ориентируемая теория и $u_{h} \in \widetilde{h}^{2}\left(\mathbb{C} P^{\infty}\right)$ - фиксированная ориентация. 
В любой $\mathbb{C}$-ориентированной теории $\left(h^{*}(\cdot), u\right)$ существуют характеристические классы Чженя-Коннера-Флойда $c f_{k}^{h}(\zeta), k=0,1, \ldots$, комплексных векторных расслоений $\zeta$. Эти классы однозначно определяются условиями: $c f_{1}^{h}(\xi)=u$, $c f_{k}^{h}(\zeta)=0$, если $k>\operatorname{dim}_{\mathbb{C}} \zeta$,

$$
c f_{k}^{h}\left(\zeta_{1}+\zeta_{2}\right)=\sum_{k_{1}+k_{2}=k} c f_{k_{1}}^{h}\left(\zeta_{1}\right) c f_{k_{2}}^{h}\left(\zeta_{2}\right) .
$$

Далее, если будет понятно о какой теории $h^{*}(\cdot)$ идет речь, мы будем писать $c f_{k}(\zeta)$ вместо $c f_{k}^{h}(\zeta)$.

Основные $\mathbb{C}$-ориентируемые теории в нашем изложении таковы: классические когомологии $H^{*}(\cdot, \mathbb{Z})$, комплексная $K$-теория $K^{*}(\cdot)$, теория комплексных кобордизмов $U^{*}(\cdot)$ и теория удвоенных комплексных кобордизмов $D U^{*}(\cdot)$. О теории $D U^{*}(\cdot)$ см. разд. 12.

В теории $U^{*}(\cdot)$ канонической считается $\mathbb{C}$-ориентация, задаваемая описанной выше универсальной $U^{*}$-ориентацией расслоения $\xi=\xi_{1}$.

Теория $D U^{*}(\cdot)$ имеет две канонические ориентации, левую $u_{l}$, соответствующую вложению $\mathbb{C} P^{\infty} \times S^{2} \rightarrow \mathbb{C} P^{\infty} \times \mathbb{C} P^{\infty}$, и правую $u_{r}$, соответствующую вложению $S^{2} \times \mathbb{C} P^{\infty} \rightarrow \mathbb{C} P^{\infty} \times \mathbb{C} P^{\infty}$ (детали см. в [17] и [36]).

В теории $H^{*}(\cdot, \mathbb{Z})$ канонической ориентацией считается $\mathbb{C}$-ориентация, задаваемая классом $u_{H} \in H^{2}\left(\mathbb{C} P^{\infty} ; \mathbb{Z}\right)$ таким, что $i_{N}^{*} u_{H}=\mathscr{D}\left\langle\mathbb{C} P^{N-1}\right\rangle$, где $\left\langle\mathbb{C} P^{N-1}\right\rangle \in$ $H_{2 N-2}\left(\mathbb{C} P^{N}\right)-$ класс гомологий, реализуемый вложением $\mathbb{C} P^{N-1} \subset \mathbb{C} P^{N}$, и $\mathscr{D}$ - классический оператор двойственности Пуанкаре.

В теории $K^{*}(\cdot)$ с кольцом скаляров $K^{*}(\mathrm{pt})=\mathbb{Z}\left[\beta^{-1}, \beta\right], \operatorname{deg} \beta=-2$, в качестве канонической $\mathbb{C}$-ориентации мы берем класс $u_{K}=(1-[\bar{\xi}]) \beta^{-1} \in \widetilde{K}^{2}\left(\mathbb{C} P^{\infty}\right)$, где $\bar{\xi}$ - расслоение, сопряженное универсальному расслоению $\xi$. Такой выбор класса $u_{K}$ мы объясним ниже.

Далеко не каждая мультипликативная теория когомологий является $\mathbb{C}$-ориентируемой. Например, не имеют $\mathbb{C}$-ориентаций: вещественная $K$-теория; теория кобордизмов $S p^{*}(\cdot)$; теория когомотопий $S^{*}(\cdot)$, задаваемая спектром сфер $S=\left\{S^{n}\right\}$

Теория $\left(U^{*}(\cdot), u\right)$ является универсальной $\mathbb{C}$-ориентированной теорией когомологий, т. е. для любой $\mathbb{C}$-ориентированной теории $\left(h^{*}(\cdot), u_{h}\right)$ существует единственное мультипликативное преобразование

$$
\mu_{h}: U^{*}(\cdot) \rightarrow h^{*}(\cdot)
$$

такое, что $\mu_{h} u=u_{h}$.

Для любой $\mathbb{C}$-ориентированной теории $\left(h^{*}(\cdot), u_{h}\right)$ множество всех мультипликативных преобразований $U^{*}(\cdot) \rightarrow h^{*}(\cdot)$ можно отождествить с множеством всех формальных рядов $\varphi\left(u_{h}\right) \in \Omega_{h}\left[\left[u_{h}\right]\right]$ вида $\varphi\left(u_{h}\right)=u_{h}+\left(u_{h}^{2}\right)$ и, следовательно, с множеством всех $\mathbb{C}$-ориентаций расслоения $\eta \rightarrow \mathbb{C} P^{\infty}$ в теории $\left(h^{*}(\cdot), u_{h}\right)$, а также с множеством всех мультипликативных преобразований $\mu_{h}^{D}: D U^{*}(\cdot) \rightarrow h^{*}(\cdot)$ таких, что $\mu_{h}^{D} u_{l}=u_{h}$ и $\mu_{h}^{D} u_{r}=\varphi\left(u_{h}\right)$.

Пусть $\left(h^{*}(\cdot), u_{h}\right)$ - некоторая $\mathbb{C}$-ориентированная теория когомологий и $\mu: U(\cdot) \rightarrow h^{*}(\cdot)$ - соответствующее мультипликативное преобразование. Тогда

$$
\mu_{h} c f_{k}(\zeta)=c f_{k}^{h}(\zeta)
$$


Например, для канонических ориентаций, описанных выше, мы получаем преобразования

$$
\mu_{H}: U^{*}(\cdot) \rightarrow H^{*}(\cdot ; \mathbb{Z}), \quad \mu_{K}: U^{*}(\cdot) \rightarrow K^{*}(\cdot), \quad \mu_{D U}^{l}, \mu_{D U}^{r}: U^{*}(\cdot) \rightarrow D U^{*}(\cdot),
$$

где $\mu_{H}$ - каноническое преобразование, соответствующее отображению спектра Тома $M U=\{M U(n)\}$ в спектр Эйленберга-Маклейна $K(\mathbb{Z})=\{K(\mathbb{Z}, n)\}$, $\mu_{K}$ - так называемое преобразование Римана-Роха-Коннера-Флойда (см. [120]), $\mu_{D U}^{l}$ и $\mu_{D U}^{r}$ соответствуют отображениям спектров Тома $M U \wedge S \rightarrow M U \wedge M U$ и $S \wedge M U \rightarrow M U \wedge M U$.

Преобразования $\mu_{H}$ и $\mu_{K}$ переводят характеристические классы ЧженяКоннера-Флойда в комплексных кобордизмах в классические классы Чженя в когомологиях и классы Чженя-Гротендика в $K$-теории.

На кольцах скаляров мы имеем:

- гомоморфизм $\mu_{H}: \Omega_{U} \rightarrow \mathbb{Z}$, где $\mu_{H}(1)=1$ и $\mu_{H}(a)=0, \operatorname{dim} a<0$, т. е. $\mu_{H}=\varepsilon-$ аугментация,

- гомоморфизм $\mu_{K}: \Omega_{U} \rightarrow \mathbb{Z}\left[\beta^{-1}, \beta\right]$ такой, что

$$
\mu_{K}\left(\left[M^{2 n}\right]\right)=\operatorname{Td}\left(M^{2 n}\right) \beta^{n},
$$

где $\operatorname{Td}\left(M^{2 n}\right) \in \mathbb{Z}$ - род Тодда стабильно комплексного многообразия. Напомним, что классический род Тодда комплексного проективного пространства $\mathbb{C} P^{n}$ равен $1, n \geqslant 0$. Для согласования рассматриваемого рода Тодда с классическим необходимо было выбрать ориентацию $u_{K}=(1-[\bar{\eta}]) \beta^{-1}$.

Пусть $\left(h^{*}(\cdot), u_{h}\right)$ - некоторая $\mathbb{C}$-ориентированная теория когомологий с кольцом скаляров $\Omega_{h}$. Каждое мультипликативное преобразование $\mu_{h}: U^{*}(\cdot) \rightarrow$ $h^{*}(\cdot)$ задает кольцевой гомоморфизм $\mu: \Omega_{U} \rightarrow \Omega_{h}$. Обратное утверждение верно лишь отчасти, так как каждый кольцевой гомоморфизм $\mu: \Omega_{U} \rightarrow \Omega_{h}$ задает мультипликативное преобразование $\mu_{h}: U^{*}(\cdot) \rightarrow h^{*}(\cdot) \otimes \Lambda=h_{\Lambda}^{*}(\cdot)$ для некоторого кольца $\Lambda$, которое в общем случае отлично от кольца $\mathbb{Z}$.

ЗАДАчи. 1. Классифицировать кольцевые гомоморфизмы $\Omega_{U} \rightarrow \Omega_{h}$, которые индуцированы мультипликативными преобразованиями $\mu_{h}$.

2. Для данного кольцевого гомоморфизма $\mu: \Omega_{U} \rightarrow \Omega_{U}$ найти минимальное кольцо $\Lambda$ такое, что кольцевой гомоморфизм $\mu \otimes 1: \Omega_{U} \rightarrow \Omega_{U} \otimes \Lambda$ поднимается до мультипликативного преобразования $U^{*}(\cdot) \rightarrow U_{\Lambda}^{*}(\cdot)$.

В [12] эти задачи были связаны с важными задачами алгебраической топологии и показано, что эффективное решение их требует формулы для логарифма формальной группы геометрических кобордизмов. Полное решение этих задач дано в [37] (см. разд. 10).

\section{6. Формальная группа геометрических кобордизмов}

С. П. Новиков в ходе выполнения работы [12] поставил задачу вычисления первого класса Чженя-Коннера-Флойда $c f_{1}\left(\xi_{1} \otimes \xi_{2}\right) \in U^{2}\left(\mathbb{C} P^{\infty} \times \mathbb{C} P^{\infty}\right)$ в виде ряда $f(u, v) \in \Omega_{U}[[u, v]]$, где $u=c f_{1}\left(\xi_{1}\right), v=c f_{1}\left(\xi_{2}\right)$. Эта задача была тесно связана с построением мультипликативных операций $\Psi_{U}^{k}$ в кобордизмах 
(см. разд. 7) - аналога важнейших операций Адамса $\Psi^{k}$ в $K$-теории и характера Чженя $\sigma_{h}: K^{*}(\cdot) \rightarrow U^{*}(\cdot) \otimes \mathbb{Q}\left(\right.$ см. разд. 9). Операции $\Psi_{U}^{k}$, известные теперь как операции Адамса-Новикова, играют существенную роль в методе С. П. Новикова вычислении группы $E_{2}^{1, *}=\operatorname{Ext}_{S}^{1, *}\left(\mathbb{Z}, \Omega_{U}\right)$ в спектральной последовательности Адамса-Новикова, а также в задачах о неподвижных точках действия конечных групп на стабильно комплексных многообразиях (см. разд. 13).

Из свойств тензорного произведения комплексных линейных расслоений непосредственно вытекает, что ряд $c f_{1}\left(\xi_{1} \otimes \xi_{2}\right)$ задает формальную группу над кольцом $\Omega_{U}$, которая была названа формальной группой геометрических кобордизмов. Приведем, следуя [12], описание ряда

$$
c f_{1}\left(\xi_{1} \otimes \xi_{2}\right)=f(u, v)=u+v+\sum a_{i, j} u^{i} v^{j},
$$

объясняющее выбор названия. Пусть $M$ - гладкое замкнутое стабильно комплексное многообразие. Обозначим через $V(M)$ группу классов эквивалентности комплексных линейных расслоений над $M$ с операцией тензорного умножения над $\mathbb{C}$. Рассмотрим отображение $f: M \rightarrow \mathbb{C P}(N), N>\operatorname{dim} M$, классифицирующее некоторое комплексное линейное расслоение $\xi \rightarrow M$. В гомотопическом классе отображения $f$ содержится отображение $f_{1}$, трансверсально регулярное на подмногообразии $\mathbb{C} P(N-1) \subset \mathbb{C} P(N)$. Стабильно комплексное подмногообразие $M_{1}=f_{1}^{-1}(\mathbb{C} P(N-1))$ называется подмногообразием, двойственным расслоению $\xi$. Так как

$$
V(M) \cong[M, \mathbb{C} P(\infty)]=H^{2}(M ; \mathbb{Z}),
$$

то сопоставление линейному расслоению $\xi$ подмногообразия $i: M_{1} \subset M$ с нормальным расслоением $i^{*} \xi$ индуцирует взаимно однозначное соответствие между множеством $V(M)$ и подмножеством в $U^{2}(M) \cong U_{m-2}(M), m=\operatorname{dim} M$, классов комплексных кобордизмов подмногообразий в $M$ коразмерности 2 , где изоморфизм устанавливается оператором двойственности Пуанкаре-Атья. Пусть $\left\{M_{1}\right\}$ и $\left\{M_{2}\right\}$ - некоторые элементы группы $V(M)$ и $\left\{M_{1}\right\} \oplus\left\{M_{2}\right\}-$ их сумма в группе $V(M)$. Тогда в группе $U^{2}(M)$ имеет место формула

$$
\left\{M_{1}\right\} \oplus\left\{M_{2}\right\}=\left\{M_{1}\right\}+\left\{M_{2}\right\}+\sum a_{i, j}\left\{M_{1}\right\}^{i}\left\{M_{2}\right\}^{j},
$$

где $\left\{M_{1}\right\}^{i} \in U^{2 i}(M)$ - класс кобордизмов двойственный $i$-й степени самопересечения многообразия $M_{1}, a_{i, j} \in \Omega_{U}^{-2(i+j-1)}-$ коэффициенты ряда $c f_{1}\left(\xi_{1} \otimes \xi_{2}\right) \in$ $\Omega_{U}[[u, v]]$.

А. С. Мищенко (в то время аспирант С. П. Новикова) рассказал о задаче вычисления ряда $c f_{1}\left(\xi_{1} \otimes \xi_{2}\right)$ алгебраисту Ю. Р. Вайнбергу, который обратил его внимание на классический результат, согласно которому если кольцо $A$ является модулем над полем рациональных чисел $\mathbb{Q}$, то любая формальная группа $F(u, v)$ над $A$ имеет вид $F(u, v)=g^{-1}(g(u)+g(v))$, где ряд $g(u)$ называется логарифмом этой формальной группы. Ряд $g^{-1}(t)$ такой, что $g^{-1}(g(u))=u$, называется экспонентой этой группы. Вскоре после этого А. С. Мищенко, используя введенные С.П. Новиковым когомологические операции в кобордизмах, нашел явное выражение для логарифма формальной группы геометрических кобордизмов. Ответ оказался удивительно красивым $[12 ;$ приложение 1 
(теорема А. С. Мищенко)]

$$
g(u)=u+\sum_{n \geqslant 1}\left[\mathbb{C} P^{n}\right] \frac{u^{n+1}}{n+1} .
$$

В работе [38] для экспоненты формальной группы геометрических кобордизмов $g^{-1}(t)$ была получена формула

$$
g^{-1}(t)=t+\sum\left[M^{2 n}\right] \frac{t^{n+1}}{(n+1) !},
$$

где класс кобордизмов $\left[M^{2 n}\right]$ однозначно определяется формулой $c f_{1}\left(\eta_{n+1}\right)=$ $\left[M^{2 n}\right] w_{n+1}$, где $\eta_{n}$ и $w_{n}$ - образующие групп $\widetilde{K}\left(S^{2 n}\right)=\mathbb{Z}$ и $H^{2 n}\left(S^{2 n} ; \mathbb{Z}\right)=\mathbb{Z}$ соответственно.

В [38] было показано, что закон сложения в формальной группе геометрических кобордизмов имеет вид

$$
f(u, v)=\frac{u+v+\sum\left[H_{i, j}\right] u^{i} v^{j}}{\mathbb{C} P(u) \mathbb{C} P(v)} .
$$

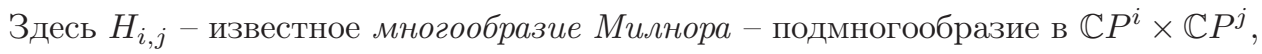
двойственное линейному расслоению $\xi_{1} \otimes \xi_{2} \rightarrow \mathbb{C} P^{i} \times \mathbb{C} P^{j}$ и $\mathbb{C} P(u)=\sum_{n \geqslant 0}\left[\mathbb{C} P^{n}\right] u^{n}$. Вывод формулы (19) опирается на формулу (16). В работах [39], [40] содержатся важные приложения формулы (19).

Весной 1967 г. С. П. Новиков был с визитом в США, где представил свою расширенную программу, включающую в том числе и приложения формальной группы геометрических кобордизмов. После его доклада на семинаре в Гарварде Дж. Тейт сказал, что он никогда не думал, что алгебраическая топология так близка к области его интересов - теории формальных групп. С. П. Новиков отметил что на его докладе присутствовал и Д. Квиллен (см. [3]).

В 1969 г. вышла работа Квиллена [41], в которой было показано, что формальную группу геометрических кобордизмов можно отождествить с универсальной формальной группой, описанной М. Лазаром в 1955 г.

Прокомментируем, следуя [37], этот результат, сыгравший важную роль в развитии теории кобордизмов и ее приложениях.

Формальная группа $\mathscr{F}(u, v)=u+v+\sum \alpha_{i, j} u^{i} v^{j}$ над коммутативным кольцом $\mathscr{A}$ называется универсальной формальной группой, если для любой формальной группы $F(u, v)$ над некоторым коммутативным кольцом $A$ существует единственный кольцевой гомоморфизм $r: \mathscr{A} \rightarrow A$ такой, что $F(u, v)=$ $u+v+\sum r\left(\alpha_{i, j}\right) u^{i} v^{j}$. Гомоморфизм $r$ называется классифицирующим. Существует прямая конструкция универсальной формальной группы $\mathscr{F}(u, v)$, из которой следует, что она единственна и определена над градуированным кольцом $\mathscr{A}=\sum_{n \geqslant 0} \mathscr{A}_{-2 n}$ (детали см. в [37]).

Лазар доказал, что $\mathscr{A}=\mathbb{Z}\left[\alpha_{n}, n=1,2, \ldots\right], \operatorname{deg} \alpha_{n}=-2 n$ (см. [42]). 
Теорема Квиллена утверждает, что гомоморфизм $r: \mathscr{A} \rightarrow \Omega_{U}$, классифицирующий формальную группу геометрических кобордизмов, является изоморфизмом (см. [41]). Перечислим факты, из сопоставления которых вытекает этот фундаментальный результат.

Доказательство мономорфности гомоморфизма $r$ извлекается из изоморфизма $\Omega_{U} \otimes \mathbb{Q} \simeq \mathbb{Q}\left[\left[\mathbb{C} P^{n}\right], n=1,2 \ldots\right]$ и формулы (17). Этот факт используется в [41].

В [11] ключевой шаг доказательства того, что кольцо $\Omega_{U}$ является кольцом полиномов, использует, что многообразия Милнора $H_{i, j}$ (см. выше) порождают все кольцо $\Omega_{U}$. Доказательство эпиморфности гомоморфизма $r$ извлекается из указанного выше свойства набора многообразий Милнора и формулы (19). В [41] эта формула не используется (по-видимому, Квиллен ее не знал).

\section{7. Операции Адамса-Новикова и степенные системы}

Операции Адамса $\Psi^{k}$ в $K$-теории сыграли ключевую роль в решении проблемы векторных полей на сферах и проблемы $J$-функтора. Эти классические проблемы алгебраической топологии были решены Ф. Адамсом в первой половине 1960-х годов. В это же время М. Атья на основе операций $\Psi^{k}$ нашел замечательное решение проблемы инварианта Хопфа 1, которую в конце 1950-х годов решил Адамс, используя свою, ставшую после этого знаменитой, спектральную последовательность.

Как уже отмечалось выше, операции Адамса-Новикова $\Psi_{U}^{k}$, введенные в [12], играют важную роль в программе С. П. Новикова.

Операции Адамса $\Psi^{k}$ в комплексной $K$-теории однозначно определяются как кольцевые гомоморфизмы

$$
\Psi^{k}: K^{0}(X) \rightarrow K^{0}(X)
$$

такие, что $\Psi^{k}[\eta]=\left[\eta^{k}\right]$ для любого линейного комплексного расслоения $\eta \rightarrow X$. Они продолжаются до мультипликативных преобразований

$$
\Psi^{k}: K^{*}(\cdot)\left[\frac{1}{k}\right] \rightarrow K^{*}(\cdot)\left[\frac{1}{k}\right],
$$

которые на кольце скаляров $K^{*}(\mathrm{pt})=\mathbb{Z}\left[\beta^{-1}, \beta\right], \operatorname{deg} \beta=-2$, действуют по формуле $\Psi^{k} \beta=k \beta$.

Операции Адамса-Новикова $\Psi_{U}^{k}, k \in \mathbb{Z}$, однозначно определяются как мультипликативные когомологические операции, которые на кольце скаляров $\Omega_{U}$ действуют по формуле $\Psi_{U}^{k} \sigma=k^{n} \sigma$, где $\sigma \in \Omega_{U}^{-2 n}$.

Из точности представления алгебры $\mathscr{A}_{U}$ на кольце $\Omega_{U}$ сразу следует, что $\Psi_{U}^{k} \Psi_{U}^{l}=\Psi_{U}^{k l}=\Psi_{U}^{l} \Psi_{U}^{k}$, и если $s \in S_{2 q}$, то $s \Psi_{U}^{k}=k^{q} \Psi_{U}^{k} s$.

Как уже отмечалось выше, любая мультипликативная операция однозначно задается своим значением на классе Тома $u=u(\xi) \in U^{2}\left(\mathbb{C} P^{\infty}\right)$. В [12] показано, что

$$
\Psi_{U}^{k} u=\frac{1}{k} u\left(\xi^{k}\right)=\frac{1}{k}[k](u), \quad k \neq 0,
$$


где ряд $[k] u=k u+\left(u^{2}\right)$ определяется индуктивно при помощи формальной группы геометрических кобордизмов $f(u, v)$ : Формальная группа $f(u, v)$ однозначно определяет ряд $\bar{u}=[-1](u)$ такой, что $f(u, \bar{u})=0$. Тогда

$$
[k](u)=f(u,[k-1](u)), \quad k \geqslant 1, \quad \text { и } \quad[-k](u)=f(\bar{u},[k-1](\bar{u})), \quad k \geqslant 2 .
$$

Из формулы $f(u, v)=g^{-1}(g(u)+g(v))$ следует, что $[k](u)=g^{-1}(k g(u)), k \in \mathbb{Z}$. Имеем $\Psi_{U}^{k} \in \mathscr{A}_{U}\left[\frac{1}{k}\right]$ и определен ряд $\Psi_{U}^{0}(u)$ как предел ряда $\frac{1}{k} g^{-1}(k g(u))$ при $k \rightarrow 0$. Получаем ряд $\Psi_{U}^{0}(u)=g(u)$, который задает проектор

$$
\Psi_{U}^{0}: U^{*}(\cdot) \otimes \mathbb{Q} \rightarrow U^{*}(\cdot) \otimes \mathbb{Q}
$$

выделяющий в теории $U^{*}(\cdot) \otimes \mathbb{Q}$ в качестве прямого слагаемого классическую теорию когомологий $H^{*}(\cdot ; \mathbb{Q})$. Развитие этого результата будет описано ниже в разд. 8.

Непосредственно из определений следует, что

$$
\mu_{K} \Psi_{U}^{k}=\Psi^{k} \mu_{K}
$$

где $\mu_{K}: U^{*}(\cdot) \rightarrow K^{*}(\cdot)$ - описанное в разд. 5 преобразование такое, что $\mu_{K} u=$ $u_{K}$.

Изучение алгебраических свойств операций $\Psi_{U}^{k}$ привело (см. [37]) к понятию формальной степенной системы.

Пусть $A$, как и выше, некоторое коммутативное кольцо с единицей. Формальной степенной системой называется совокупность

$$
\left\{f_{k}(u) \in A[[u]], k \in \mathbb{Z}\right\}
$$

такая, что $f_{k}(0)=0$ и $f_{k}\left(f_{l}(u)\right)=f_{k l}(u)$.

Скажем, что система $\left\{f_{k}(u)\right\}$ имеет тип $s$, если для любого $k$ ряд $f_{k}(u)$ имеет вид

$$
f_{k}(u)=k^{s} u+\sum \mu_{i}(k) u^{i+1}, \quad \mu_{i}(k) \in A .
$$

Число $s$ всегда предполагается положительным. Ясно, что любая система $\left\{f_{k}(u)\right\}$ типа $s$ содержит подсистему $\left\{\hat{f}_{k}(u)=f_{k^{q}}(u)\right\}$ типа qs для фиксированного натурального $q$. Не всякая степенная система имеет тип $s$, например, степенной системой является совокупность $\left\{f_{k}(u)=u^{k^{2}}\right\}$.

В [37] доказано, что для любой формальной степенной системы $\left\{f_{k}(u)\right\}$ типа $s$ существует ряд $B(u) \in A \otimes \mathbb{Q}[[u]]$, не зависящий от $k$, такой, что

$$
f_{k}(u)=B^{-1}\left(k^{s} B(u)\right), \quad \text { где } \quad B^{-1}(B(u))=u .
$$

Ряд $B(u)$ однозначно определяется степенной системой и называется ее логарифмом, а ряд $B^{-1}(u)$ называется ее экспонентой.

Любая формальная группа $F(u, v)$ над $A$ задает степенную систему

$$
\left\{f_{k}(u)=[k](u), k \in \mathbb{Z}\right\},
$$


где $[0](u)=0$ и $[k](u)=F(u,[k-1](u))$. Эта система имеет тип 1 , она содержит подсистемы $\left\{\hat{f}_{k}(u)=f_{k^{s}}(u)\right\}$ типа $s$ для любого натурального $s$.

Обозначим через $\Omega(s) \subset \Omega_{U}$ подкольцо, порожденное всеми коэффициентами формальных рядов

$$
k^{s} \Psi^{k^{s}}(u)=\left[k^{s}\right](u)=k^{s} u+\sum \mu_{i}^{(s)}(k) u^{i+1} \in \Omega_{U}[[u]]
$$

по всем $k$.

$$
\begin{aligned}
& \text { Имеем } \Omega(s)=\sum_{n \geqslant 0} \Omega(s)_{-2 n} \text {. В [37] отмечено, что } \\
& \quad \min \left\{s_{n}\left(\left[M^{2 n}\right]\right),\left[M^{2 n}\right] \in \Omega(s)_{-2 n}\right\}=\text { НОД }\left\{k^{s}\left(k^{n s}-1\right), k=2,3, \ldots\right\},
\end{aligned}
$$

и поэтому кольцо $\Omega(s)$ не совпадает с кольцом $\Omega_{U}$ ни для какого $s$, но

$$
\Omega(s) \otimes \mathbb{Q}=\Omega_{U} \otimes \mathbb{Q} .
$$

Зафиксируем изоморфизм $\Omega_{U} \simeq \mathbb{Z}\left[a_{n}\right]$ и рассмотрим мультипликативный проектор $\pi_{p}: \Omega_{U} \rightarrow \Omega_{U}$, такой что $\pi_{p} a_{n}=a_{n}$, если $n=p^{i}-1$, и $\pi_{p} a_{n}=0$ в остальных случаях. Тогда гомоморфизм $\pi_{p}: \Omega(1) \rightarrow \pi_{p}\left(\Omega_{U}\right)$ является эпиморфизмом.

Формальная степенная система $f_{U}=\left\{\left[k^{s}\right](u)\right\}$ над кольцом $\Omega(s)$ является универсальной формальной степенной системой типа $s$ на категории колеи, без кручения, т. е. для любой формальной степенной системы $\left\{f_{k}(u)\right\}$ типа $s$ над любым коммутативным кольцом $A$ без кручения существует единственный кольцевой гомоморфизм $\phi: \Omega(s) \rightarrow A$ такой, что $f=\phi\left[f_{U}\right]$.

Из существования логарифма $B(u)$ степенной системы вытекает, что каждой формальной степенной системе над кольцом $A$ без кручения соответствует формальная группа

$$
F(u, v)=B^{-1}(B(u)+B(v)) \in A \otimes \mathbb{Q}[[u, v]] .
$$

Пусть $\phi: \Omega(s) \rightarrow A$ - гомоморфизм, классифицирующий степенную систему $f=\left\{f_{k}(u)\right\}$ типа $s$ над $A$. Для того чтобы эта степенная система порождалась формальной группой над кольцом $A$, необходимо и достаточно, чтобы гомоморфизм $\phi$ продолжался до гомоморфизма $\hat{\phi}: \Omega_{U} \rightarrow A$, т. е. $\left.\hat{\phi}\right|_{\Omega(s)}=\phi$.

Из теоремы Лазара вытекает, что любая формальная группа $F(u, v)$ над любым коммутативным кольцом $A$ является образом формальной группы (например, универсальной) над кольцом без кручения.

В [37] построено континуальное семейство формальных степенных систем над кольцом $\mathbb{Z} / p$, не являющихся образом никакой степенной системы над кольцом без кручения.

Операция $[k](u)=u \oplus \cdots \oplus u$, где $\oplus-$ сложение в формальной группе, играет важную роль в классической теории формальных групп. В случае универсальной формальной группы она, как показано выше, задает универсальные степенные системы $\left\{\left[k^{s}\right](u)\right\}$ типа $s$ над кольцами без кручения. 


\section{8. Характеры Чженя и Чженя-Дольда}

Классическая $K$-теория $K^{*}(\cdot)$ пришла в алгебраическую топологию из алгебраической геометрии [43]. Большую роль в $K$-теории играет характер Чженя $\mathrm{ch}: K^{*}(X) \rightarrow H^{*}(X ; \mathbb{Q})$. Характер Чженя ch является мультипликативным функториальным преобразованием, которое однозначно определяется тем, что для одномерного комплексного расслоения $\xi \rightarrow X$ имеет место формула $\operatorname{ch} \xi=\exp c_{1}(\xi)$, где $c_{1}(\xi) \in H^{2}(X ; \mathbb{Z})$ - первый класс Чженя в когомологиях.

А. Гротендик дал следующее обобщение теоремы Римана-Роха-Хирцебруха.

Пусть $f: X \rightarrow Y$ - проективное отображение гладких алгебраических многообразий. Тогда для любого элемента $x \in K^{0}(X)$ имеет место формула

$$
\operatorname{ch}\left(f_{!} x\right) \operatorname{Td}\left(\tau_{Y}\right)=f_{*}\left(\operatorname{ch}(x) \operatorname{Td}\left(\tau_{X}\right)\right),
$$

где $\tau_{X}$ и $\tau_{Y}$ - касательные расслоения и $\operatorname{Td}\left(\tau_{X}\right), \operatorname{Td}\left(\tau_{Y}\right)-$ их классы Тодда.

Отображение гладких многообразий $f: X \rightarrow Y$ называется $c_{1}$-отображением, если существует класс $c_{1} \in H^{2}(X ; \mathbb{Z})$ такой, что $w_{2}(X)-f^{*} w_{2}(Y) \equiv c_{1} \bmod 2$, где $w_{2}(\cdot)$ - второй класс Штифеля-Уитни. В [30] показано, что приведенная выше формула верна для всех $c_{1}$-отображений.

В этом разделе мы основное внимание уделим характеру Чженя-Дольда $\mathrm{ch}_{U}$ в комплексных кобордизмах. Аналог теоремы Римана-Роха-Хирцебруха-Гротендика для отображений $f: X \rightarrow Y$ стабильно комплексных многообразий и преобразования $\mathrm{ch}_{U}$ обсудим в разд. 14.

В [12] было введено мультипликативное функториальное преобразование характер Чженя в кобордизмах

$$
\sigma \mathrm{h}: \widehat{K}^{*}(X) \rightarrow U^{*}(X) \otimes \mathbb{Q},
$$

где $\widehat{K}^{*}(\cdot)=K^{0}(\cdot)+K^{1}(\cdot)-2$-периодическая комплексная $K$-теория. Преобразование $\sigma \mathrm{h}$ однозначно определяется тем, что для одномерного комплексного расслоения $\xi \rightarrow X$ имеет место формула

$$
\sigma \mathrm{h} \xi=\exp g\left(c f_{1}(\xi)\right),
$$

где $g(u)$ - логарифм формальной группы геометрических кобордизмов и $c f_{1}(\xi)-$ первый класс Чженя-Коннера-Флойда. В [12] подчеркивается, что построение характера Чженя в кобордизмах - одна из важных задач, решаемых на основе логарифма формальной группы геометрических кобордизмов.

Теория $\widehat{K}(\cdot)$ имеет мультипликативный класс Тома $u_{\widehat{K}}(\zeta)$, универсальный для всех комплексных расслоений, который однозначно определяется условием $u_{\widehat{K}}(\xi)=c f_{1}^{\widehat{K}}(\xi)=[1-\bar{\xi}]$, где $\xi \rightarrow X-$ одномерное расслоение. Имеет место мультипликативное преобразование $\mathrm{Td}: U^{*}(\cdot) \rightarrow \widehat{K}(\cdot)$ такое, что $\mathrm{Td} u(\zeta)=$ $u_{\widehat{K}}(\zeta)$. При этом преобразовании $\mathrm{Td}\left[\mathbb{C} P^{n}\right]=1, n \geqslant 0$. Следовательно,

$$
\operatorname{Td} \sigma \mathrm{h} \xi=\exp (-\ln (1-\operatorname{Td} u(\xi)))=\xi .
$$

Таким образом, композиция преобразований

$$
\widehat{K}^{*}(X) \stackrel{\sigma \mathrm{h}}{\rightarrow} U^{*}(X) \otimes \mathbb{Q} \stackrel{\mathrm{Td}}{\rightarrow} \widehat{K}^{*}(X) \otimes \mathbb{Q}
$$

совпадает с каноническим гомоморфизмом, индуцированным вложением $\mathbb{Z} \subset \mathbb{Q}$. 
Характером Чженя-Дольда в теории $h^{*}(\cdot)$ с $\Omega_{h}=h^{*}(\mathrm{pt})$ называется преобразование теорий когомологий

$$
\operatorname{ch}_{h}: h^{*}(\cdot) \rightarrow H^{*}\left(\cdot ; \Omega_{h} \otimes \mathbb{Q}\right) ; \quad H^{i}\left(\cdot ; \Omega_{h} \otimes \mathbb{Q}\right)=\sum H^{l}\left(\cdot ; \Omega_{h}^{i-l} \otimes \mathbb{Q}\right),
$$

которое для $X=$ pt представляет собой канонический гомоморфизм $\Omega_{h} \rightarrow$ $\Omega_{h} \otimes \mathbb{Q}$, где $H^{*}(\cdot)$ - классические когомологии.

Теорема существования и единственности характера Чженя-Дольда доказана впервые Дольдом (см. [44]). В случае, когда $h^{*}(\cdot)$ - комплексная $K$-теория, характер Чженя-Дольда $\mathrm{ch}_{h}$ совпадает с классическим характером Чженя ch (см. [44]). Этим объясняется выбор названия и обозначения для отображения $\mathrm{ch}_{h}$ (см. [38]).

Для любой обобщенной теории гомологий $h_{*}(\cdot)=\sum h_{n}(\cdot)$ существует аналогично определяемый гомологический характер Чженя-Дольда

$$
\operatorname{ch}^{h}: h_{*}(\cdot) \rightarrow H_{*}\left(\cdot ; \Omega_{h} \otimes \mathbb{Q}\right) .
$$

Для теории стабильных гомотопий $\pi_{*}^{s}$, где $\Omega_{\pi_{*}^{s}}-$ кольцо стабильных гомотопических групп сфер и, следовательно, $\Omega_{\pi_{*}^{s}} \otimes \mathbb{Q}=\pi_{0}^{s} \otimes \mathbb{Q}=\mathbb{Q}$, преобразование $\mathrm{ch}_{\pi_{*}^{s}}$ разлагается в композицию с классическим гомоморфизмом Гуревича $H: \pi_{*}^{s}(\cdot) \rightarrow H_{*}(\cdot ; \mathbb{Z})$, который соответствует отображению спектров $\mu_{s}^{H}: S \rightarrow K(\mathbb{Z})$ такому, что $\mu_{s}^{H}(1)=1 \in \widetilde{H}_{0}\left(S^{0}\right)$, где $1 \in \pi_{0}^{s}\left(S^{0}\right)$.

Подчеркнем, что характеры Чженя-Дольда $\mathrm{ch}_{h}$ и $\mathrm{ch}^{h}$ существуют для любых теорий когомологий $h^{*}(\cdot)$ и гомологий $h_{*}(\cdot)$. Мультипликативность теории $h^{*}(\cdot)$ или $\mathbb{C}$-ориентируемость этой теории не требуется.

Перечислим основные свойства характера Чженя-Дольда $\mathrm{ch}_{h}$.

а) Для любого конечного комплекса $X$ гомоморфизм $\operatorname{ch}_{h} \otimes \mathbb{Q}: h^{*}(X) \otimes \mathbb{Q} \rightarrow$ $H^{*}\left(X ; \Omega_{h} \otimes \mathbb{Q}\right)$ является изоморфизмом.

b) Для любой мультипликативной теории когомологий $h^{*}(\cdot)$ характер ЧженяДольда $\mathrm{ch}_{h}$ является кольцевым гомоморфизмом.

Пусть $A^{h}$ - кольцо стабильных когомологических операций в теории $h^{*}(\cdot)$. Используя действие кольца $A^{h}$ на $\Omega_{h}$, введем в кольце $H^{*}\left(X ; \Omega_{h} \otimes \mathbb{Q}\right)$ структуру $A^{h}$-модуля.

c) Характер Чженя-Дольда $\mathrm{ch}_{h}$ является гомоморфизмом $A^{h}$-модулей.

d) Пусть $\varphi: h_{1}(\cdot) \rightarrow h_{2}(\cdot)$ - преобразование теории когомологий. Тогда $\operatorname{ch}_{h_{2}} \varphi=\varphi \operatorname{ch}_{h_{1}}$.

Доказательство этих свойств легко следует из конструкции характера Чженя-Дольда, данной в [44].

В качестве следствия мы получаем следующее утверждение.

Пусть $h_{1}^{*}(\cdot)$ и $h_{2}^{*}(\cdot)$ - теории когомологий и $\lambda: \Omega_{h_{1}} \rightarrow \Omega_{h_{2}} \otimes \mathbb{Q}$ - гомоморфизм скаляров. Тогда для любого конечного клеточного комплекса $X$ имеет место преобразование

$$
\operatorname{ch}_{h_{1}, \lambda}^{h_{2}}: h_{1}^{*}(X) \rightarrow h_{2}^{*}(X) \otimes \mathbb{Q}
$$

однозначно определяемое условием $\operatorname{ch}_{h_{2}} \operatorname{ch}_{h_{1}, \lambda}^{h_{2}}=\lambda \operatorname{ch}_{h_{1}}$. 
Таким образом, для любого кольцевого гомоморфизма $\lambda: \Omega_{U} \rightarrow \mathbb{Q}$ получается преобразование

$$
\operatorname{ch}_{U, \lambda}^{\widehat{K}}: U^{*}(\cdot) \rightarrow \widehat{K}^{*}(\cdot) \otimes \mathbb{Q},
$$

которое в работах И. М. Кричевера [45], [46] нашло важное приложение в задаче о действиях групп на стабильно комплексных многообразиях.

Преобразование $\mathrm{ch}_{h_{1}, \lambda}^{h_{2}}$ определено и для бесконечных клеточных комплексов $X$ с конечномерными остовами таких, что $H^{*}(X ; \mathbb{Z})$ не имеет кручения.

Для любой мультипликативной теории когомологий $h^{*}(\cdot)$ определено мультипликативное преобразование $\operatorname{ch}_{\widehat{K}, 1}^{h}: \widehat{K}^{*}(\cdot) \rightarrow h^{*}(\cdot) \otimes \mathbb{Q}$, где $1: \mathbb{Z} \rightarrow \Omega_{h}^{0} \subset \Omega_{h}-$ гомоморфизм, задаваемый единицей кольца $\Omega_{h}$. Это преобразование в [38] названо характером Чженя в теории $h^{*}(\cdot)$. Преобразование $\operatorname{ch}_{\widehat{K}, 1}^{U}$ совпадает с характером Чженя в кобордизмах $\sigma \mathrm{h}, \mathrm{cm} .(20)$.

Введем кольцо $\Omega_{U}(\mathbb{Z})=\sum_{n \geqslant 0} \Omega_{U}^{-2 n}(\mathbb{Z})$. Здесь

$$
\Omega_{U}^{-2 n}(\mathbb{Z})=\left\{\sigma \in \Omega_{U}^{-2 n} \otimes \mathbb{Q}: s \sigma \in \Omega_{U}^{0}=\mathbb{Z} \text { для } s \in S_{2 n}\right\},
$$

где $S=\sum_{n \geqslant 0} S_{2 n}-$ алгебра Ландвебера-Новикова. В [38] доказано, что

$$
\Omega_{U}(\mathbb{Z}) \simeq \mathbb{Z}\left[\frac{\left[\mathbb{C} P^{n}\right]}{n+1}, n=1,2, \cdots\right] .
$$

Таким образом, $g(u) \in \Omega_{U}(\mathbb{Z})[[u]]$ и, следовательно, $g^{-1}(t) \in \Omega_{U}(\mathbb{Z})[[t]]$. Ясно, что кольцо $\Omega_{U}(\mathbb{Z})$ замкнуто относительно действия алгебры $\mathscr{A}_{U}$, индуцированного вложением $\Omega_{U}(\mathbb{Z}) \subset \Omega_{U} \otimes \mathbb{Q}$. Имеет место изоморфизм $\Omega_{U}(\mathbb{Z}) \cong S^{*}$, при котором действие алгебры Ландвебера-Новикова $S$ на $\Omega_{U}(\mathbb{Z})$ соответствует каноническому левому действию $l$ алгебры Хопфа $S$ на двойственной алгебре $S^{*}$ (детали см. в разд. 12).

Характер Чэсеня-Долъда в кобордизмах (см. [38]) разлагается в композицию

$$
\operatorname{ch}_{U}: U^{*}(X) \stackrel{\widehat{c h}_{U}}{\longrightarrow} H^{*}\left(X ; \Omega_{U}(\mathbb{Z})\right) \stackrel{j}{\rightarrow} H^{*}\left(X ; \Omega_{U} \otimes \mathbb{Q}\right),
$$

где $j$ - преобразование, индуцированное вложением $j: \Omega_{U}(\mathbb{Z}) \rightarrow \Omega_{U} \otimes \mathbb{Q}$. Преобразование $\widehat{c h}_{U}: U^{*}(\cdot) \rightarrow H^{*}\left(\cdot ; \Omega_{U}(\mathbb{Z})\right)$ называется приведенным характером Чженя-Дольда, как мультипликативное преобразование оно однозначно определяется (см. разд. 5) тем, что для одномерного комплексного расслоения $\xi \rightarrow X$ имеет место формула $\operatorname{ch}_{U} c f_{1}(\xi)=g^{-1}\left(c_{1}(\xi)\right)$.

Имеет место соответствующее преобразование теорий гомологий

$$
\widehat{\mathrm{ch}}^{U}: U_{*}(\cdot) \rightarrow H_{*}\left(\cdot ; \Omega_{U}(\mathbb{Z})\right) .
$$

Используя канонические изоморфизмы $\Omega_{U}(\mathbb{Z}) \simeq S_{*} \simeq H_{*}(M U ; \mathbb{Z})$ преобразования $\widehat{c h}_{U}$ и $\widehat{c h}^{U}$ можно отождествить с преобразованиями, задаваемыми отображениями спектров $S \wedge M U \rightarrow K(\mathbb{Z}) \wedge M U$. В некоторых работах преобразование $\widehat{c h}^{U}$ называется гомоморфизмом Гуревича, по аналогии с классическим гомоморфизмом Гуревича $H: \pi_{*}^{s}(X) \rightarrow H_{*}(X ; \mathbb{Z})$, задаваемым вложением $S \rightarrow K(\mathbb{Z})$. 
Преобразования $\mu_{H}: U^{*}(\cdot) \rightarrow H^{*}(\cdot, \mathbb{Z})$ и $\mu^{H}: U_{*}(\cdot) \rightarrow H_{*}(\cdot, \mathbb{Z})$ задаются отображением спектров $M U \rightarrow K(\mathbb{Z})$ (см. разд. 5). Ясно, что аугментация $\varepsilon: \Omega_{U}(\mathbb{Z}) \rightarrow$ $\mathbb{Z}: \varepsilon(1)=1, \varepsilon(\sigma)=0, \operatorname{deg} \sigma<0$ переводит $\widehat{c h}_{U}$ в $\mu_{H}$ и $\widehat{c h}^{U}$ в $\mu^{H}$. Вычисление образа гомоморфизма $\mu^{H}: U_{*}(X) \rightarrow H_{*}(X, \mathbb{Z})$ называется проблемой Стинрода реализации циклов образами фундаментальных циклов стабильно комплексных многообразий. Эта проблема эквивалентна проблеме дифференциалов в спектральной последовательности Атья-Хирцебруха в теории $U_{*}(\cdot)$. Так как кольцо $\Omega_{U}$ не имеет кручения, то преобразования $\mu_{H}$ и $\mu^{H}$ являются эпиморфизмами для комплексов $X$ без кручения в $\mathbb{Z}$-гомологиях (см. разд. 9).

ТЕОРЕмА ЦЕЛОчИСЛЕНности приведенного характера Чженя-Дольда [38]: Имеет место функториальное мультипликативное преобразование $\mathscr{A}_{U}$-модулей

$$
\widehat{c h}_{U}: U^{*}(X) \otimes_{\Omega_{U}} \Omega_{U}(\mathbb{Z}) \rightarrow H^{*}\left(X ; \Omega_{U}(\mathbb{Z})\right),
$$

которое является изоморфизмом для пространств $X$ без кручения в гомологиях.

При $X=\mathbb{C} P^{\infty}$ мы получаем изоморфизм $\mathscr{A}_{U}$-модулей

$$
\widehat{c h}_{U}: \Omega_{U}(\mathbb{Z})[[u]] \rightarrow \Omega_{U}(\mathbb{Z})[[t]]
$$

такой, что $\widehat{c h}_{U}(g(u))=t$ и $\widehat{c h}_{U} u=g^{-1}(t)$, где $u \in U^{2}\left(\mathbb{C} P^{\infty}\right), t \in H^{2}\left(\mathbb{C} P^{\infty} ; \mathbb{Z}\right)$,

$$
g(u)=u+\sum\left[\mathbb{C} P^{n}\right] \frac{u^{n+1}}{n+1}, \quad g^{-1}(t)=t+\sum\left[M^{2 n}\right] \frac{t^{n+1}}{(n+1) !} .
$$

Для любой операции $s \in S, s \neq 1$, по построению, st $=0$ и, следовательно, $s g(u)=0$.

Для $u \in U^{2}\left(\mathbb{C} P^{\infty}\right)$ мы имеем $s u=0$, если $s \neq s_{n}$ и $s_{n} u=u^{n+1}$, и, следовательно, $s g^{-1}(t)=0$, если $s \neq s_{n}$ и $s_{n} g^{-1}(t)=\left(g^{-1}(t)\right)^{n+1}$.

Используя эти факты, можно непосредственно вывести формулы действия операторов $s \in S$ на коэффициенты $\left[\mathbb{C} P^{n}\right]$ и $\left[M^{2 n}\right], n=1,2, \ldots$, рядов $g(u)$ и $g^{-1}(t)$ и получить канонические изоморфизмы

$$
\Omega_{U}(\mathbb{Z}) \simeq \mathbb{Z}\left[\frac{\left[\mathbb{C} P^{1}\right]}{2}, \ldots, \frac{\left[\mathbb{C} P^{n}\right]}{n+1}, \ldots\right] \simeq \mathbb{Z}\left[\frac{\left[M^{2}\right]}{2 !}, \ldots, \frac{\left[M^{2 n}\right]}{(n+1) !}, \ldots\right]
$$

с алгеброй Хопфа $S_{*}$, двойственной алгебре Ландвебера-Новикова $S$.

В качестве следствия получаем следующее утвреждение.

Теорема ЕДИнственности. Пусть $\phi: U^{*}(\cdot) \rightarrow H^{*}\left(\cdot, \Omega_{U}(\mathbb{Z})\right)$ - нетривиальное мультипликативное преобразование теорий когомологий, коммутирующее с действием алгебры Ландвебера-Новикова S. Тогда ф совпадает с приведенным характером Чженя-Дольда $\widehat{c h}_{U}$.

СИЛЬНАЯ ТЕОРЕМА ЦЕЛОЧИСЛЕННОСТИ. Приведенный характер ЧженяДольда $\widehat{\mathrm{ch}}_{U}$ для $X=S^{2 n}$ разлагается в композицию

$$
\widehat{\operatorname{ch}}_{U}: U^{*}\left(S^{2 n}\right) \rightarrow H^{*}\left(S^{2 n} ; \Omega_{U}\right) \rightarrow H^{*}\left(S^{2 n} ; \Omega_{U}(\mathbb{Z})\right) .
$$


Соответствующий результат для классического характера Чженя $\mathrm{ch}=\mathrm{ch}_{K}$ вытекает из теоремы периодичности Ботта в комплексной $K$-теории. Теоремы целочисленности для характера Чженя ch, основанные на теореме Ботта, были получены Ф. Адамсом (см. [47]). В частности, построен инвариант Адамса $e: \pi_{2 n-1}^{s} \rightarrow \mathbb{Q} / \mathbb{Z}$, дающий сильную оценку снизу на порядок группы $J_{2 n-1} \subset \pi_{2 n-1}^{s}$, где $J_{n}=J\left(S^{n}\right)$ - образ стабильного $J$-гомоморфизма Уайтхеда $J: \pi_{n}(S O) \rightarrow \pi_{n}^{s}$, где $S O=\lim S O(n)$ (см. [48]).

Приведем конструкцию инварианта

$$
e_{U}: \pi_{2 n-1}^{s} \rightarrow \Omega_{U}^{-2 n}(\mathbb{Z}) / \Omega_{U}^{-2 n}, \quad n \geqslant 1,
$$

- аналога инварианта Адамса в случае комплексных кобордизмов.

Выберем представителя $f: S^{2(N+n)-1} \rightarrow S^{2 N}$ элемента $[f] \in \pi_{2 n-1}^{s}$. Положим $X_{f}=S^{2 N} \cup_{f} D^{2(N+n)}$. Для пары $\left(X_{f}, S^{2 N}\right)$ имеет место точная последовательность $\mathscr{A}_{U}$-модулей

$$
0 \rightarrow \widetilde{U}^{*}\left(S^{2(N+n)}\right) \stackrel{\pi^{*}}{\rightarrow} U^{*}\left(X_{f}\right) \stackrel{i^{*}}{\rightarrow} U^{*}\left(S^{2 N}\right) \rightarrow 0 .
$$

Обозначим через $v_{N} \in U^{2 N}\left(X_{f}\right)$ такой элемент, что $i^{*} v_{N}=u_{N}$, где $u_{N}$ - канонический образующий, соответствующий вложению $S^{2 N} \subset M U(N)$. Тогда $\operatorname{ch}_{U} v_{N}=x_{N}+\sigma \pi^{*} \operatorname{ch}_{U} u_{N+n}$, где $\sigma \in \Omega_{U}^{-2 n}(\mathbb{Z})$ и $i^{*} x_{N}=\operatorname{ch}_{U} u_{N}$.

Образ $[\sigma]$ элемента $\sigma$ при проекции $\Omega_{U}^{-2 n}(\mathbb{Z}) \rightarrow \Omega_{U}^{-2 n}(\mathbb{Z}) / \Omega_{U}^{-2 n}$ не зависит от выбора представителя класса $[f]$ и элемента $v_{n}$. По определению $e_{U}(f)=[\sigma]$. Стандартно проверяется, что отображение $e_{U}$ является гомоморфизмом. При $n=1$, используя кольцо $U^{*}\left(\mathbb{C} P^{2}\right)$, получаем изоморфизм

$$
e_{U}: \pi_{1}^{s}=\mathbb{Z} / 2 \rightarrow \Omega_{U}^{-2}(\mathbb{Z}) / \Omega_{U}^{-2}=\mathbb{Z} / 2
$$

который переводит образующий элемент в $\frac{1}{2}\left[\mathbb{C} P^{1}\right]$, а при $n=2$, используя кольцо $U^{*}\left(\mathbb{H} P^{2}\right)$, получаем эпиморфизм

$$
e_{U}: \pi_{3}^{s}=\mathbb{Z} / 8+\mathbb{Z} / 3 \rightarrow \Omega_{U}^{-4}(\mathbb{Z}) / \Omega_{U}^{-4}=\mathbb{Z} / 4+\mathbb{Z} / 3
$$

который переводит образующие элементы в $\frac{1}{4}\left[\mathbb{C} P^{1}\right]^{2}$ и $\frac{1}{3}\left[\mathbb{C} P^{2}\right]$ соответственно.

Доказательство этих утверждений следует из формул для $\operatorname{ch}_{U} f_{1}(\xi)$ и $\operatorname{ch}_{U} f_{2}(\zeta)$ (см. ниже), где $\xi \rightarrow \mathbb{C} P^{\infty}$ и $\zeta \rightarrow \mathbb{H} P^{\infty}$ - универсальные расслоения.

Скажем, что элемент $a \in U^{k}(X)$ является $\operatorname{ch}_{U}$-целочисленным, если $\operatorname{ch}_{U} a \in$ $H^{*}\left(X ; \Omega_{U}\right) \subset H^{*}\left(X ; \Omega_{U}(\mathbb{Z})\right)$. Элемент $a \in U^{k}(X)$ называется косферическим, если существует отображение $f: \Sigma^{2 N-k} X \rightarrow S^{2 N}$ такое, что $f^{*} u_{N}=S^{2 N-k} a$.

Из сильной теоремы целочисленности вытекает, что любой косферический элемент является $\mathrm{ch}_{U}$-целочисленным. Пространство $X$ называется $\mathrm{ch}_{U}$-целочисленным, если его характер Чженя-Дольда разлагается в композицию

$$
\operatorname{ch}_{U}: U^{*}(X) \rightarrow H^{*}\left(X ; \Omega_{U}\right) \rightarrow H^{*}\left(X ; \Omega_{U}(\mathbb{Z})\right) .
$$

В качестве следствия мы получаем, что если группа $H^{*}(X ; \mathbb{Z})$ не имеет кручения и $X$ является $\mathrm{ch}_{U}$-целочисленным, то характер Чженя-Дольда задает изоморфизм $\mathscr{A}_{U}$-модулей $U^{*}(X) \rightarrow H^{*}\left(X ; \Omega_{U}\right)$. 
Используя теорему Джеймса [49] о гомотопической эквивалентности

$$
\Sigma \Omega X \stackrel{\sim}{\rightarrow} \bigvee_{n \geqslant 1} X^{(n)},
$$

где $\Omega X$ - пространство петель над $X$ и $X^{(n)}=X \wedge \cdots \wedge X-$ приведенная $n$-я степень пространства $X$, мы получаем, что для любого $\mathrm{ch}_{U}$-целочисленного пространства $X$ его пространство петель также является $\mathrm{ch}_{U}$-целочисленным.

Обратное утверждение неверно, например, пространство $S^{1} \simeq \Omega \mathbb{C} P^{\infty}$ является $\mathrm{ch}_{U}$-целочисленным, а пространство $\mathbb{C} P^{\infty}$ таковым не является.

Интересно рассмотреть более подробно случаи $X=S^{2 k}, k \geqslant 1$. Положим $Y_{k}=\Omega \Sigma S^{2 k}$. Согласно теореме Джеймса, $\Sigma Y_{k} \simeq \Sigma\left(\bigvee_{q \geqslant 1} S^{2 k q}\right)$. Следовательно, в качестве образующих свободного $\Omega_{U}$-модуля $U^{*}\left(Y_{k}\right)$ можно выбрать косферические классы $v_{k q} \in U^{2 k q}\left(Y_{n}\right), q \geqslant 0$, где $v_{0}=1 \in \Omega_{U}$ и $v_{k}^{q}=q$ ! $v_{k q}$. В случае $k=1$ имеет место отображение $\varphi: Y_{1}=\Omega S^{3} \rightarrow \mathbb{C} P^{\infty}$, задающее образующую группы $H^{2}\left(Y_{1} ; \mathbb{Z}\right)=\mathbb{Z}$. Используя тот факт, что $s_{n} u=u^{n+1}, n \geqslant 0$, где $u=c f_{1}(\xi)$ и $\xi \rightarrow \mathbb{C} P^{\infty}$ - универсальное линейное расслоение, получаем формулу:

$$
\varphi^{*} u=v_{1}+\sum_{q \geqslant 1}\left[M^{2 q}\right] v_{q+1}=g^{-1}\left(v_{1}\right)
$$

где $\left[M^{2 q}\right]$ - коэффициенты разложения в ряд экспоненты $g^{-1}(u)$ формальной группы геометрических кобордизмов.

Рассмотрим универсальное одномерное кватернионное расслоение $\zeta \rightarrow \mathbb{H} P^{\infty}$. Имеет место формула (см. [37])

$$
\operatorname{ch}_{U} c f_{2}(\zeta)=\tau+\sum_{q \geqslant 1} a_{q}\left[N^{4 q}\right] \frac{\tau^{q+1}}{(2 q+2) !}
$$

где $a_{q}=2$, если $q$ нечетно, и $a_{q}=1$, если $q$ четно. Здесь $\tau$ - образующая группы $H^{4}\left(\mathbb{H} P^{\infty} ; \mathbb{Z}\right)=\mathbb{Z}$ и $N^{4 q}$ - стабильно комплексное многообразие с родом Тодда, равным 1.

Допустим, что над $Y_{2}=\Omega S^{5}$ определено некоторое одномерное кватернионное расслоение $\eta \rightarrow Y_{2}$ с $c f_{2}(\eta)=m w_{2}$, где $w_{2}$ - образующая группы $H^{4}\left(Y_{2} ; \mathbb{Z}\right)=\mathbb{Z}$. Тогда существует непрерывное отображение $\varphi: Y_{2} \rightarrow \mathbb{H} P^{\infty}$ такое, что $\varphi^{*} \tau=m w_{2}$. Имеем:

$$
c f_{2}(\eta)=m v_{2}+\sum_{q \geqslant 1} \alpha_{q} v_{2 q+2}, \quad \alpha_{q} \in \Omega_{U}^{-4 q} .
$$

Ввиду косферичности пространства $Y_{2}$

$$
\operatorname{ch}_{U} c f_{2}(\eta)=m w_{2}+\sum_{q \geqslant 1} \alpha_{q} w_{2 q+2},
$$

где $w_{2 q}=\operatorname{ch}_{U} v_{2 q}-$ образующие групп $H^{2 q}\left(Y_{2} ; \mathbb{Z}\right)=\mathbb{Z}$. 
Таким образом, из условий

$$
\varphi^{*} \zeta=\eta, \quad \operatorname{Td}\left(N^{4 q}\right)=1 \quad \text { и } \quad w_{2}^{q}=q ! w_{2 q+2}
$$

мы получаем соотношение

$$
\operatorname{Td} \alpha_{q}=a_{q} \frac{(q+1) !}{(2 q+2) !} m^{q+1} .
$$

Например, $\mathrm{Td} \alpha_{1}=m^{2} / 3$ !, Td $\alpha_{2}=m^{3} / 5$ !. Ясно, что для данного $m \neq 0$ числа $\operatorname{Td} \alpha_{q}$ не могут быть целыми для всех $q$. Таким образом, $m=0$ для любого одномерного кватернионного расслоения $\eta \rightarrow Y_{2}$.

Пусть $M$ - некоторый топологический моноид. Используя, что $\Omega \Sigma X$ гомотопически эквивалентно универсальному моноиду пространства $X$ с отмеченной точкой $*$, мы получаем, что любое непрерывное отображение $\varphi: X \rightarrow M$ продолжается до отображения $\Omega \Sigma X \rightarrow M$. Для $\mathrm{ch}_{U}$-целочисленного пространства $X$ можно в ряде случаев показать, что для данного пространства $Y$ отображение $\varphi: X \rightarrow Y$ не продолжается до отображения $\Omega \Sigma X \rightarrow Y$ и тем самым доказать, что не существует топологического моноида $M$, гомотопически эквивалентного пространству $Y$.

Выше мы рассмотрели случай $X=S^{4}$ и $Y=\mathbb{H} P^{\infty}$. Аналогично, можно использовать $X=S^{2 k}$ и получить условия, при которых отображения $S^{2 k} \rightarrow Y$ не продолжаются до отображений $\Omega \Sigma S^{2 k} \rightarrow Y$.

В последние годы развиваются теории квантовых когомологий и квантовых комплексных кобордизмов. В [50] получены результаты на основе соответствующего аналога характера Чженя-Дольда.

\section{9. Спектральная последовательность Атья-Хирцебруха}

Для любого клеточного пространства $X$ и любой теории когомологий $h^{*}(\cdot)$ существует спектральная последовательность Атья-Хирцебруха

$$
\mathrm{AH}_{h}(X)=\left\{E_{r}^{s}(X)=\sum E_{r}^{s, t}, r \geqslant 1 ; d_{r}^{s, t}: E_{r}^{s, t} \rightarrow E_{r}^{s+r, t-r+1}\right\}
$$

отвечающая фильтрации $\left\{\right.$ pt $\left.\subset X_{1} \subset \cdots \subset X_{q} \subset \cdots \subset X\right\}$, где $X_{q}-q$-мерный остов в $X$, и связывающая теорию $h^{*}(\cdot)$ с классическими когомологиями $H^{*}(\cdot)$.

$\mathrm{B} \mathrm{AH}_{h}(X)$ мы имеем $E_{2}^{s, t}(X)=H^{s}\left(X, h^{t}(\mathrm{pt})\right)$, и если $X-n$-мерный клеточный комплекс, то

$$
E_{\infty}^{s, t}(X)=h_{s}^{s+t}(X) / h_{s+1}^{s+t}(X), \quad \text { где } \quad h_{q}^{m}(X)=\operatorname{ker}\left(h^{m}(X) \rightarrow h^{m}\left(X_{q-1}\right)\right) .
$$

Для любой теории гомологий $h_{*}(X)$ определена соответствующая гомологическая спектральная последовательность Атья-Хирцебруха

$$
A H^{h}(X)=\left\{E_{s}^{r}(X)=\sum E_{s, t}^{r}, r \geqslant 1 ; d_{s, t}^{r}: E_{s, t}^{r} \rightarrow E_{s-r, t+r-1}^{r}\right\}
$$

такая, что $E_{s, t}^{2}(X)=H_{s}\left(X, h_{t}(\mathrm{pt})\right)$ и

$$
E_{s, t}^{\infty}(X)=h_{s+t}^{s+1}(X) / h_{s+t}^{s}(X), \quad \text { где } \quad h_{m}^{q}(X)=\operatorname{Im}\left(h_{m}\left(X_{q-1}\right) \rightarrow h_{m}(X)\right) .
$$


Положим $X_{s}=\varnothing$ при $s<0$. Рассмотрим пары троек

$$
\left(X_{s+r}, X_{s}, X_{s-r}\right) \subset\left(X_{s+2 r-1}, X_{s+r-1}, X_{s-1}\right)
$$

и вложения

$$
i_{s, r}:\left(X_{s}, X_{s-r}\right) \rightarrow\left(X_{s+r-1}, X_{s-1}\right) .
$$

Имеет место коммутативная диаграмма

$$
\begin{array}{ccc}
h^{s+t}\left(X_{s+r-1}, X_{s-1}\right) & \stackrel{\left(i_{s, t}^{*}\right)^{s+t}}{\longrightarrow} & h^{s+t}\left(X_{s}, X_{s-r}\right) \\
\downarrow_{s+r-1, r} & \downarrow \delta_{s, r} \\
h^{s+t+1}\left(X_{s+2 r-1}, X_{s+r-1}\right) & \stackrel{\left(i_{s+t, r}^{*}\right)^{s+t}}{\longrightarrow} h^{s+t+1}\left(X_{s+r}, X_{s}\right)
\end{array}
$$

в которой $\delta_{s, r}-$ граничный гомоморфизм точной последовательности тройки $\left(X_{s+r}, X_{s}, X_{s-r}\right)$. Для $\mathrm{AH}_{h}(X)$ имеют место следующие утверждения:

$-E_{r}^{s, t}=\operatorname{Im}\left(i_{s, r}^{*}\right)^{s+t}$

$-d_{r}^{s, t}=\bar{\delta}_{s, r}: \operatorname{Im}\left(i_{s, r}^{*}\right)^{s+t} \rightarrow \operatorname{Im}\left(i_{s+t, r}^{*}\right)^{s+t}$

$-\operatorname{ker} d_{r}^{s, t}=\operatorname{Im}\left(h^{s+t}\left(X_{s+r}, X_{s-1}\right) \rightarrow h^{s+t}\left(X_{s}, X_{s-r}\right)\right)$;

- проекция $\pi: \operatorname{ker} d_{r}^{s, t} \rightarrow E_{r+1}^{s, t}=\operatorname{ker} d_{r}^{s, t} / \operatorname{Im} d_{r}^{s-r, t+r-1}$ определяется композицией

$$
h^{s+t}\left(X_{s+r}, X_{s-1}\right) \stackrel{j_{1}}{\rightarrow} h^{s+t}\left(X_{s}, X_{s-r}\right) \stackrel{j_{2}}{\longrightarrow} h^{s+t}\left(X_{s}, X_{s-r-1}\right),
$$

т. е. $\operatorname{Im} j_{1} \rightarrow \operatorname{Im} j_{2} j_{1}$.

Член $E_{1}^{s, t}(X)$ в $\mathrm{AH}_{h}(X)$ совпадает с группой $s$-мерных коцепей $C^{s}\left(X, h^{t}(\mathrm{pt})\right)$, а дифференциал $d_{1}^{s, t}$ представляет собой обычный кограничный оператор

$$
\delta: C^{s}\left(X, h^{t}(\mathrm{pt})\right) \rightarrow C^{s+1}\left(X, h^{t}(\mathrm{pt})\right)
$$

и, следовательно, $E_{2}^{s, t}=H^{s}\left(X, h^{t}(\mathrm{pt})\right)$.

Если $h^{*}(\cdot)$ - мультипликативная теория когомологий, то спектральная последовательность $\mathrm{AH}_{h}(\cdot)$ является мультипликативной, т. е. ее члены $E_{r}^{*, *}$ являются градуированными косокоммутативными кольцами, дифференциалы $d_{r}-$ дифференцированиями, член $E_{r+1}$ - кольцом когомологий члена $E_{r}$, умножение в $E_{2}$ совпадает с обычным умножением в когомологиях с коэффициентами в кольце $h^{*}(\mathrm{pt})$. Если $X-$ конечный клеточный комплекс, то имеет место изоморфизм $E_{\infty}^{s, t}(X)=h_{s}^{s+t}(X) / h_{s+1}^{s+t}(X)$ и умножение в $E_{\infty}^{* * *}$ индуцируется умножением в кольце $h^{*}(X)$. Здесь $h_{s}^{q}(X)=\operatorname{ker}\left(h^{q}(X) \rightarrow h^{q}\left(X_{s-1}\right)\right)$. В ряде случаев удается доказать изоморфизм $E_{\infty}^{s, t}(X)=h_{s}^{s+t}(X) / h_{s+1}^{s+t}(X)$ и для бесконечных клеточных комплексов $X$ с конечномерными остовами $X_{n}$. В этом случае говорят, что $\mathrm{AH}_{h}(X)$ сильно сходится.

Любое непрерывное отображение $f: X \rightarrow Y$ клеточных пространств гомотопически эквивалентно клеточному отображению, поэтому определен гомоморфизм

$$
f^{*}: \mathrm{AH}_{h}(Y) \rightarrow \mathrm{AH}_{h}(X)
$$


Любое преобразование теорий когомологий $\mu: h_{1}^{*}(\cdot) \rightarrow h_{2}^{*}(\cdot)$ задает гомоморфизм

$$
\mathrm{AH}_{\mu}: \mathrm{AH}_{h_{1}}(X) \rightarrow \mathrm{AH}_{h_{2}}(X)
$$

для любого клеточного пространства $X$.

Возьмем в качестве $h_{2}^{*}(\cdot)$ теорию когомологий $H^{*}\left(\cdot ; \Omega_{h} \otimes \mathbb{Q}\right)$ и рассмотрим отображение спектральных последовательностей Атья-Хирцебруха, задаваемое характером Чженя-Дольда. Отображение

$$
\operatorname{AH}_{\mathrm{ch}_{h}}: E_{2, h}^{*, *}(X)=H^{*}\left(X ; \Omega_{h}\right) \rightarrow E_{2, h_{2}}^{*, *}(X)=H^{*}\left(X ; \Omega_{h} \otimes \mathbb{Q}\right)
$$

соответствует гомоморфизму $\Omega_{h} \rightarrow \Omega_{h} \otimes \mathbb{Q}$. Спектральная последовательность $\mathrm{AH}_{h_{2}}(X)$ тривиальна, т. е. все ее дифференциалы $d_{r}, r \geqslant 2$, тривиальны. Таким образом, спектральная последовательность $\mathrm{AH}_{h} \otimes \mathbb{Q}$ тривиальна. Приведем следствия этого результата.

- $\mathrm{B} \mathrm{AH}_{h}(X)$ все дифференциалы имеют конечный порядок.

- Пусть $\Omega_{h}$ не имеет элементов конечного порядка. Тогда если $H^{*}(X ; \mathbb{Z})$ не имеет элементов конечного порядка, то спектральная последовательность $\mathrm{AH}_{h}(X)$ тривиальна.

Аналогичные утверждения имеют место и для гомологической спектральной последовательности $\mathrm{AH}^{h}(X)$.

Алгебра стабильных когомологических операций $\mathscr{A}_{h}$ действует на $\mathrm{AH}_{h}(\cdot)$ таким образом, что (см. [51]):

- каждая операция $a \in \mathscr{A}_{h}$ определяет гомоморфизмы $E_{r}^{*, *}(X) \rightarrow E_{r}^{*, *}(X)$, $r \geqslant 2$,

- операция $a: E_{r}(X) \rightarrow E_{r}(X)$ является естественной относительно отображений $f: X \rightarrow Y$,

- в члене $E_{2}^{*, *}(X)=H^{*}\left(X, h^{*}(\mathrm{pt})\right)$ операция $а$ индуцирована действием операции $a: h^{*}(\mathrm{pt}) \rightarrow h^{*}(\mathrm{pt})$,

- действие в $E_{r+1}(X)$ индуцировано действием в $E_{r}(X)$,

- в $E_{\infty}(X)$ действие присоединено к действию $a: h^{*}(X) \rightarrow h^{*}(X)$.

Таким образом, если операция $a \in \mathscr{A}_{h}$ тривиально действует на $h^{*}(\mathrm{pt})$, то она тривиально действует на $\mathrm{AH}_{h}(X)$ для любого $X$.

В случаях комплексной $K$-теории $K^{*}(\cdot)$ и теории комплексных кобордизмов $U^{*}(\cdot)$ представления алгебр $\mathscr{A}_{K}$ и $\mathscr{A}_{U}$ на кольцах $K^{*}(\mathrm{pt})=\mathbb{Z}\left[\beta, \beta^{-1}\right]$ и $U^{*}(\mathrm{pt})=\Omega_{U}$ являются точными и несут важную информацию о дифференциалах в $\mathrm{AH}_{K}(\cdot)$ и $\mathrm{AH}_{U}(\cdot)$ (см. [24], [51]).

Следуя [51], введем $\mathscr{A}_{h}$-модуль дифференциала $d_{r}^{t}=\sum d_{r}^{s, t}$ в $\mathrm{AH}_{h}(\cdot)$ как градуированный $\mathscr{A}_{h}$-модуль $\mathscr{D}_{r}^{t}$ с одной образующей $d_{r}^{t}, \operatorname{deg} d_{r}^{t}=0$, и соотношениями $a d_{r}^{t}=0$, где $a \in \mathscr{A}_{h}$, которые имеют место тогда и только тогда, когда для любого пространства $X$ и элемента $x \in E_{r}^{*, *}(X)$ оператор $a: E_{r}(X) \rightarrow E_{r}(X)$ аннулирует элемент $d_{r}^{t} x \in E_{r}^{*, t-r+1}(X)$.

Рассмотрим левые $\mathscr{A}_{h}$-идеалы

$$
\mathscr{A}_{h}(n)=\left\{a \in \mathscr{A}_{h}: a(\sigma)=0 \text { для всех } \sigma \in h^{n}(\mathrm{pt})\right\} .
$$


В [51] доказано, что если для стабильного спектра $Y$, представляющего данную теорию $h^{*}(\cdot)$, спектральная последовательность $\mathrm{AH}_{h}(Y)$ тривиальна, то $\mathscr{D}_{r}^{t}=$ $\mathscr{A}_{h} / J_{r}^{t}$, где $J_{r}^{t}$ - идеал, порожденный идеалами $\mathscr{A}_{h}(t)$ и $\mathscr{A}_{h}(t-r+1)$.

Требуемыми свойствами обладают спектры, представляющие теории кобордизмов $S O^{*}(\cdot), U^{*}(\cdot), \mathscr{D} U^{*}(\cdot), S p^{*}(\cdot)$ и $S^{*}(\cdot)$.

В качестве нетривиального следствия этого результата, в [51] и [24] доказано, что в теории комплексных кобордизмов для любого $r$ имеет место изоморфизм

$$
\operatorname{Ext}_{\mathscr{A}_{U}}^{1,2 r}\left(\mathscr{D}_{r}^{0}, \Omega_{U}\right) \simeq \operatorname{Ext}_{\mathscr{A}_{U}}^{1,2 r}\left(\Omega_{U}, \Omega_{U}\right) .
$$

Порядком ord $d_{r}^{s, t}$ дифференциала $d_{r}^{s, t}$ называется наименьшее число, аннулирующее элементы $d_{r}^{s, t} x$, где $x \in E_{r}^{s, t}(X)$ для любого пространства $X$.

Используя вычисление группы $\operatorname{Ext}_{\mathscr{A}_{U}}^{1,2 r}\left(\Omega_{U}, \Omega_{U}\right)$ (см. [12]) получаем: ord $d_{3}^{0}=2$, $\operatorname{ord} d_{5}^{0}=12$ и ord $d_{2 r+1}^{0}=$ НОД $\left\{k^{N}\left(k^{r}-1\right), k=2,3, \ldots\right\}$ для $r>2$.

Естественно возник вопрос [24], каким условиям должна удовлетворять теория $h^{*}(\cdot)$, чтобы имела место формула

$$
\operatorname{ord} d_{2 r+1}^{0}=\operatorname{ord}\left|\operatorname{Ext}_{\mathscr{A}_{h}}^{1,2 r}(\Lambda, \Lambda)\right|,
$$

где $\Lambda=h^{*}(\mathrm{pt})$.

Например, обычные когомологии $H^{*}(\cdot)$ и теория когомотопий $S^{*}(\cdot)$ в отличие от теории $U^{*}(\cdot)$ этим свойством не обладают.

Спектральные последовательности Атья-Хирцебруха являются эффективным средством решения задач об ориентируемости векторного расслоения в данной теории когомологий и задач о реализуемости циклов и коциклов.

Пусть $\zeta \rightarrow X$ - некоторое $n$-мерное векторное расслоение над клеточным пространством, ориентируемое в классическом смысле. Фиксируем класс его ориентации $u_{H} \in H^{n}(T \zeta ; \mathbb{Z})$. Для данной теории когомологий $h^{*}(\cdot)$ в $\mathrm{AH}_{h}(T \zeta)$ определен элемент $\left[u_{H}\right] \in H^{n}\left(T \zeta ; h^{0}(\mathrm{pt})\right)=E_{2}^{n, 0}(T \zeta)$. Непосредственно из определений получаем, что расслоение $\zeta \rightarrow X$ является $h^{*}$-ориентируемым тогда и только тогда, когда $\left[u_{H}\right]$ является циклом всех дифференциалов в $\mathrm{AH}_{h}(T \zeta)$.

Рассмотрим некоторую подгруппу $G_{n}$ группы $S O(n)$ и $n$-мерное векторное расслоение $\zeta_{n} \rightarrow B G_{n}$, соответствующее вложению $G_{n} \subset S O(n)$. Получаем, что универсальная ориентация для класса векторных $G_{n}$-расслоений существует тогда и только тогда, когда $\left[u_{H}\left(\zeta_{n}\right)\right] \in E_{2}^{n, 0}\left(T \zeta_{n}\right)$ является циклом всех дифференциалов в $\mathrm{AH}_{h}(T \zeta)$.

Для любых теорий бордизмов и кобордизмов ставятся задачи реализации циклов и коциклов. Обсудим эти задачи для теорий $U_{*}(\cdot)$ и $U^{*}(\cdot)$.

Говорят, что класс $a \in H_{m}(X ; \mathbb{Z})$ реализуем в теории $U_{*}(\cdot)$, если существуют стабильно комплексное многообразие $M^{m}$ и отображение $f: M^{m} \rightarrow X$ такое, что $f_{*}\left\langle M^{m}\right\rangle=a$, где $\left\langle M^{m}\right\rangle \in H_{m}\left(M^{m} ; \mathbb{Z}\right)$ - фундаментальный класс многообразия $M^{m}$. Класс $a \in H_{m}(X ; \mathbb{Z})$ реализуем в теории $U_{*}(\cdot)$ тогда и только тогда, когда элемент $[a] \in E_{m, 0}^{2}(X)$ является циклом всех дифференциалов в $\mathrm{AH}_{U_{*}}(X)$.

Говорят, что класс $a \in H^{k}(X ; \mathbb{Z})$ реализуем в теории $U^{*}(\cdot)$, если существует отображение $f: \Sigma^{2 N-k} X \rightarrow M U(N)$ такое, что $f^{*} u_{N}=\Sigma^{2 N-k} a$. Класс $a \in$ 
$H^{k}(X ; \mathbb{Z})$ реализуем в теории $U^{*}(\cdot)$ тогда и только тогда, когда элемент $[a] \in$ $E_{2}^{k, 0}(X)$ является циклом всех дифференциалов в $\mathrm{AH}_{U^{*}}(X)$.

Пусть теперь $X=W^{l} / \partial W^{l}$, где $W^{l}$ - стабильно комплексное многообразие с границей и $a \in H^{k}\left(W^{l}, \partial W^{l} ; \mathbb{Z}\right)$. Согласно $H^{*}(\cdot)$-двойственности Пуанкаре однозначно определен класс гомологий $D a \in H_{l-k}\left(W^{l} ; \mathbb{Z}\right)$. Непосредственно из конструкции геометрического оператора $U^{*}(\cdot)$-двойственности Пуанкаре получаем, что класс $a$ является $U^{*}$-реализуем тогда и только тогда, когда $U_{*}$-реализуем класс $D a$.

В алгебраической геометрии хорошо известна гипотеза Ходжа. Приведем ее целочисленный вариант.

Пусть $X$ - гладкое проективное многообразие. Тогда каждый элемент группы $H^{2 k}(X ; \mathbb{Z}) \cap H^{k, k}(X)$, где $H^{k, k}(X)$ - когомологии Ходжа алгебраического многообразия $X$, является классом когомологий некоторого алгебраического цикла. Атья и Хирцебрух предъявили первый контрпример к этой гипотезе (см. [52]). Для этого они ввели спектральную последовательность в $K$-теории, получившую название спектральной последовательности Атья-Хирцебруха. Результат вытекал из построения многообразия $X$ и элемента $a \in H^{2 k}(X ; \mathbb{Z}) \cap$ $H^{k, k}(X)$ конечного порядка такого, что элемент $[a] \in E_{2}^{2 k, 0}(X)$ не является циклом первого нетривиального дифференциала $d_{3}$ в $\operatorname{AH}_{K}(X)$.

Формулы для дифференциалов в спектральной последовательности АтьяХирцебруха в теориях $U^{*}(\cdot)$ и $K^{*}(\cdot)$, использующие когомологические операции Стинрода, и приложения этих формул получены в [24], [51].

Результаты по приложению теории комплексных кобордизмов к задачам алгебраической геометрии, связанным с целочисленной гипотезой Ходжа, представлены в [53].

\section{0. Проекторы в теории кобордизмов}

Мультипликативное преобразование $\mu: U^{*}(\cdot) \rightarrow U^{*}(\cdot)$ является проектором $($ т. е. $\mu \mu=\mu)$ тогда и только тогда, когда $\mu u=\mu(u)=u+\sum \varphi_{k} u^{k+1}$, где $\varphi_{k} \in \Omega_{U}$ и $\mu \varphi_{k}=0$ для всех $k \geqslant 1$.

Большой запас проекторов в теории $U^{*}(\cdot)$ дает конструкция операторов деления, предложенная С. П. Новиковым в [12].

Пусть $\Lambda$ - некоторое коммутативное кольцо с единицей без элементов конечного порядка и без делителей нуля. Обозначим через $h_{\Lambda}^{*}(\cdot)$ теорию $h^{*}(\cdot) \otimes \Lambda$.

Пусть $y \in \Omega_{U}^{-2 n}, n>0$. Оператором деления на $y$ называется когомологическая операция

$$
\Delta_{y}: U_{\Lambda}^{*}(\cdot) \rightarrow U_{\Lambda}^{*}(\cdot)
$$

такая, что $\Delta_{y} y=1$ и

$$
\Delta_{y} a b=\left(\Delta_{y} a\right) b+a\left(\Delta_{y} b\right)-y\left(\Delta_{y} a\right)\left(\Delta_{y} b\right)
$$

для любых $a$ и $b$ из $U_{\Lambda}^{*}(X)$. 
Из определения следует, что если оператор $\Delta_{y}$ существует для данного $y \in$ $\Omega_{U}$, то у является мультипликативно неразложимым в кольце $\Omega_{U}$. Напомним, что это условие эквивалентно условию $s_{n} y \neq 0$ (об операциях $s_{n}$ см. разд. 2).

Имеет место следующий результат.

Пусть $y \in \Omega_{U}^{-2 n}, n>0$ и $s_{n} y=-d \neq 0$. Возьмем $\Lambda=\mathbb{Z}\left[d^{-1}\right]$. Тогда

1. Множество операторов деления на $y$ можно отождествить с множеством всех мультипликативных операций $\mu: U_{\Lambda}^{*}(\cdot) \rightarrow U_{\Lambda}^{*}(\cdot)$ таких, что

$$
\mu u=\varphi_{y}(u)=u+y u^{n}\left(d^{-1} u+\left(u^{2}\right)\right) .
$$

2. Каждая мультипликативная операция $\mu: U_{\Lambda}^{*}(\cdot) \rightarrow U_{\Lambda}^{*}(\cdot)$ такая, что $\mu u=$ $\varphi_{y}(u)=u+y u^{n}\left(d^{-1} u+\left(u^{2}\right)\right)$, является проектором и выделяет в теории $U_{\Lambda}^{*}(\cdot)$ теорию когомологий $U_{y}^{*}(\cdot)$ с кольцом скаляров $\Omega_{U, y}=\Lambda\left[a_{1}, \ldots, a_{n-1}, a_{n+1}, \ldots\right]$.

В качестве следствия получаем:

1. Существует мультипликативный проектор $\mu: U^{*}(\cdot) \rightarrow U^{*}(\cdot)$, образом которого является $\mathbb{C}$-ориентированная теория когомологий с кольцом скаляров $\mathbb{Z}\left[a_{n}\right]$, где $n$ пробегает числа вида $p^{q}-1$ для всех простых чисел $p$ и $q=1,2, \ldots$.

2. Фиксируем простое число $p$ и рассмотрим кольцо целых $p$-адических чисел $\mathbb{Z}_{p}$. Выберем классы $\left[\mathbb{C} P^{n}\right.$ ] для $n \neq p^{q}-1$ в качестве мультипликативных образующих кольца $\Omega_{U} \otimes \mathbb{Z}_{p}$. Тогда существует мультипликативный проектор $\mu_{p}: U^{*}(\cdot) \otimes \mathbb{Z}_{p} \rightarrow U^{*}(\cdot) \otimes \mathbb{Z}_{p}$ такой, что $\mu_{p}\left[\mathbb{C} P^{n}\right]=0, n \neq p^{q}-1$. Образом этого проектора является $\mathbb{C}$-ориентированная теория когомологий с кольцом скаляров $\mathbb{Z}_{p}\left[a_{p^{q}-1}\right], q=1,2, \ldots$.

Алгебраическая теория операторов деления и ее топологические приложения были развиты в [54].

Используя классическую теорию препятствий в гомотопической топологии, Э. Браун и $Ф$. Петерсон в 1966 г. [55] доказали, что спектр $M U \mathbb{Z}_{p}$ расщепляется в прямую сумму сдвигов некоторого спектра, получившего название $c n e \kappa-$ тра Брауна-Петерсона и обозначение $B P_{p}$. Вскоре после этого С. П. Новиков в [12] получил результат Брауна-Петерсона при помощи введенных им операторов деления на скаляры (см. выше). В вычислениях гомотопических групп методом спектральной последовательности Адамса-Новикова он существенно использовал спектр Брауна-Петерсона. Для этого он ввел и исследовал алгебру когомологических операций $\mathscr{A}_{B P}$ в теории $B P^{*}(\cdot)$, определяемой этим спектром.

Важным приложением теоремы Квиллена об универсальности формальной группы геометрических кобордизмов явилось построение проекторов, получивших его имя.

До работы [41] не удавалось получить каноническое (универсальное) расщепление спектра $M U \mathbb{Z}_{p}$. Такое расщепление было получено на основе теории формальных групп.

Формальные группы $F_{1}(x, y)$ и $F_{2}(x, y)$ над некоторым коммутативным кольцом $A$ называются сильно изоморфными над $A$, если существует ряд $\varphi(x)=$ $x+\left(x^{2}\right) \in A[[x]]$ такой, что

$$
\varphi\left(F_{1}(x, y)\right)=F_{2}(\varphi(x), \varphi(y)) .
$$


Например, логарифм $g(x)$ формальной группы $F(x, y)=F_{1}(x, y)$ над $A \otimes \mathbb{Q}$ задает ее сильный изоморфизм с аддитивной группой $F_{2}(x, y)=x+y$.

Квиллен применил теорему Картье о том, что над кольцом $\mathscr{A} \otimes \mathbb{Z}_{p}$ универсальная формальная группа $\mathscr{F}(x, y)$ сильно изоморфна группе $\mathscr{F}_{p}(x, y)$ с логарифмом

$$
g_{p}(u)=u+\sum\left[\mathbb{C} P^{p^{q}-1}\right] \frac{u^{p^{q}}}{p^{q}} .
$$

Проекторы $\pi_{p}$ однозначно определяются условиями

$$
\pi_{p}\left(\left[\mathbb{C} P^{p^{q}-1}\right]\right)=\left[\mathbb{C} P^{p^{q}-1}\right] \quad \text { и } \quad \pi_{p}\left(\left[\mathbb{C} P^{n}\right]\right)=0, \quad n \neq p^{q}-1 .
$$

Из теоремы Картье следует, что кольцевой гомоморфизм

$$
\pi_{p}: \mathbb{Z}\left[\left[\mathbb{C} P^{n}\right], n \geqslant 1\right] \rightarrow \mathbb{Z}\left[\left[\mathbb{C} P^{n}\right], n \geqslant 1\right]
$$

однозначно продолжается до проектора

$$
\widehat{\pi}_{p}: \Omega_{U} \otimes \mathbb{Z}_{p} \rightarrow \Omega_{U} \otimes \mathbb{Z}_{p}, \quad \widehat{\pi}_{p} \widehat{\pi}_{p}=\widehat{\pi}_{p}
$$

Ряд $u_{B P}=u_{B P}(u)$, задающий сильный изоморфизм группы $\mathscr{F}(x, y)$ с группой $\mathscr{F}_{P}(x, y)$, определяет проектор

$$
\pi_{p}: U^{*}(\cdot) \otimes \mathbb{Z}_{p} \rightarrow U^{*}(\cdot) \otimes \mathbb{Z}_{p},
$$

выделяющий в $U^{*}(\cdot) \otimes \mathbb{Z}_{p}$ теорию когомологий Брауна-Петерсона $B P^{*}(\cdot)$. В [41] приведена формула, описывающая средствами теории формальных групп ряд $u_{B P}(u)$.

В [41] дано описание алгебры $\mathscr{A}_{B P}$ всех когомологических операций в теории Брауна-Петерсона, которое теперь используется в приложениях спектральной последовательности Адамса-Новикова. Алгебра $\mathscr{A}_{B P}$ является свободным модулем над кольцом скаляров $\Omega_{B P}=\mathbb{Z}_{p}\left[a_{p-1}, \ldots, a_{p^{q}-1}, \ldots\right]$ с аддитивным базисом операций, определяемых характеристическими классами Чженя-КоннераФлойда в теории $B P^{*}(\cdot)$. Обратим внимание, что группа, порожденная над $\mathbb{Z}_{p}$ этими базисными операциями, имеет структуру коалгебры, но, в отличие от случая Ландвебера-Новикова, не является алгеброй Хопфа, так как композиция базисных операций Квиллена разлагается по базису с коэффициентами из кольца $\Omega_{B P}$, а не из $\mathbb{Z}_{p}$.

Построение проекторов Квиллена - это важный пример решения задач, сформулированных в разд. 4. Опишем общий случай.

Рассмотрим некоторую $\mathbb{C}$-ориентированную теорию когомологий $\left(h^{*}(\cdot), u_{h}\right)$. Как отмечено выше, существует мультипликативное преобразование $\mu_{h}: U^{*}(\cdot) \rightarrow$ $h^{*}(\cdot)$, переводящее формальную группу геометрических кобордизмов $f(u, v)=$ $u+v+\sum a_{i j} u^{i} v^{j}$ в формальную группу над кольцом $\Omega_{h}$

$$
f_{h}(x, y)=x+y+\sum \alpha_{i j} x^{i} y^{j}
$$

где $x=\mu_{h} u, y=\mu_{h} v, \alpha_{i j}=\mu_{h}\left(a_{i j}\right)$. 
Из универсальности формальной группы $f(u, v)$ над $\Omega_{U}$ следует, что множество формальных групп над $\Omega_{h}$ можно отождествить с множеством кольцевых гомоморфизмов $\Omega_{U} \rightarrow \Omega_{h}$. Используя этот факт, получаем следующее утверждение.

Формальная группа $F(x, y)$ над $\Omega_{h}$ сильно изоморфна формальной группе $f_{h}(x, y)$ тогда и только тогда, когда кольцевой гомоморфизм $\varphi: \Omega_{U} \rightarrow \Omega_{h}$, классифицирующий формальную группу $F(x, y)$, продолжается до мультипликативного преобразования $\mu_{h}(F): U^{*}(\cdot) \rightarrow h^{*}(\cdot)$.

Для доказательства этого результата ориентацию $u_{h} \in h^{2}\left(\mathbb{C} P^{\infty}\right)$ надо заменить на ориентацию $\varphi\left(u_{h}\right)$, где $\varphi\left(u_{h}\right)$ - ряд, задающий сильный изоморфизм групп $f_{h}(x, y)$ и $F(x, y)$.

Приведем приложение этого результата к описанным выше каноническим ориентациям.

1. Рассмотрим классические когомологии $\left(H^{*}(\cdot ; \Lambda), u_{H}\right)$ с коэффициентами в коммутативном кольце $\Lambda$. Тогда $f_{H}(x, y)=x+y$, и мы получаем, что множество всех мультипликативных преобразований

$$
\mu: U^{*}(\cdot) \rightarrow H^{*}(\cdot ; \Lambda)
$$

можно отождествить с множеством всех формальных групп над $\Lambda$, сильно изоморфных линейной группе.

2. Рассмотрим комплексную $K$-теорию $\left(K_{\Lambda}^{*}(\cdot), u_{K}\right)$ с коэффициентами в $\Lambda$. Тогда $f_{K}(x, y)=x+y-\beta x y$, и мы получаем, что множество всех мультипликативных преобразований

$$
\mu: U^{*}(\cdot) \rightarrow K_{\Lambda}^{*}(\cdot)
$$

можно отождествить с множеством всех формальных групп над $\Lambda$, сильно изоморфных группе $x+y-\beta x y$.

3. Множество всех мультипликативных преобразований

$$
\mu: U^{*}(\cdot) \rightarrow U_{\Lambda}^{*}(\cdot)
$$

можно отождествить с множеством всех формальных групп над $\Omega_{U} \otimes \Lambda$, сильно изоморфных универсальной формальной группе. па

Например, операции $\Psi_{U}^{k}: U^{*}(\cdot) \rightarrow U^{*}(\cdot)\left[\frac{1}{k}\right]$ соответствует формальная груп-

$$
F(u, v)=u+v+\sum k^{i+j-1} a_{i j} u^{i} v^{j} .
$$

Ряд $\Psi_{U}^{k} u=\frac{1}{k}[k](u)$ задает сильный изоморфизм формальной группы геометрических кобордизмов с этой группой.

Рассмотрим ряд $g(u)=u+\sum\left[\mathbb{C} P^{n}\right] \frac{u^{n+1}}{n+1}$ и кольцевой гомоморфизм $\mu$ : $\Omega_{U} \rightarrow \Lambda$. Положим

$$
g_{\mu}(x)=x+\sum \mu\left(\left[\mathbb{C} P^{n}\right]\right) \frac{x^{n+1}}{n+1} .
$$

В качестве следствия предыдущих результатов получаем решение задач из разд. 4. 
1. Кольцевой гомоморфизм $\mu: \Omega_{U} \rightarrow \Lambda$ продолжается до мультипликативного преобразования $\mu: U^{*}(\cdot) \rightarrow H^{*}(\cdot ; \Lambda)$ тогда и только тогда, когда $g_{\mu}(x) \in$ $\Lambda[[x]]$, где $x=\mu_{H} u$.

2. Кольцевой гомоморфизм $\mu: \Omega_{U} \rightarrow \Lambda[\beta]$ продолжается до мультипликативного преобразования $\mu: U^{*}(\cdot) \rightarrow K_{\Lambda}(\cdot)$ тогда и только тогда, когда $\exp \left(\beta g_{\mu}(x)\right) \in \Lambda[\beta][[x]]$.

3. Кольцевой гомоморфизм $\mu: \Omega_{U} \rightarrow \Omega_{U} \otimes \Lambda$ продолжается до мультипликативного преобразования $\mu: U^{*}(\cdot) \rightarrow U_{\Lambda}^{*}(\cdot)$ тогда и только тогда, когда $g^{-1}\left(g_{\mu}(x)\right) \in \Omega_{U} \otimes \Lambda[[x]]$.

В [56] дано описание проекторов в $U^{*}$-теории, связанных с $S U^{*}$-теорией. Эти проекторы нашли приложение в задаче о деформации умножения в $U^{*}$-теории (см. [54]).

\section{1. Двузначные формальные группы}

Изучение формальных степенных систем в [37] во многом было стимулировано тем, что топологические конструкции приводят к другим реализациям универсальных степенных систем, определяемым многозначными формальными группами, первые важные примеры которых были введены в [37].

Пусть $F(u, v)$ - формальная группа над кольцом $A$ и $\bar{u}=[-1](u)-$ ряд, задающий обратный элемент для операции $u \oplus v=F(u, v)$. Положим $x=u \bar{u}=$ $|u|^{2} \in A[[u]]$. Тогда

$$
|F(u, v)|^{2}=F(u, v) F(\bar{u}, \bar{v}) \quad \text { и } \quad|F(\bar{u}, v)|^{2}=F(\bar{u}, v) F(u, \bar{v}) .
$$

В [37] показано, что формальные ряды $|F(u, v)|^{2}+|F(\bar{u}, v)|^{2}$ и $|F(u, v)|^{2}|F(\bar{u}, v)|^{2}$ из кольца $A[[u, v]]$ принадлежат подкольцу $A[[x, y]] \subset A[[u, v]]$, следовательно, их можно переписать в виде $\Theta_{1}(x, y)$ и $\Theta_{2}(x, y)$ соответственно.

Рассмотрим над кольцом $A[[x, y]]$ квадратное уравнение

$$
Z^{2}-\Theta_{1}(x, y) Z+\Theta_{2}(x, y)=0
$$

и ряд $B(x)=x+\left(x^{2}\right)$, который в кольце $A \otimes \mathbb{Q}[[u]]$ имеет вид $g(u) g(\bar{u})=-g(u)^{2}$. Тогда решения

$$
\begin{aligned}
& |F(u, v)|^{2}=B^{-1}\left((\sqrt{B(x)}+\sqrt{B(y)})^{2}\right), \\
& |F(\bar{u}, v)|^{2}=B^{-1}\left((\sqrt{B(x)}-\sqrt{B(y)})^{2}\right)
\end{aligned}
$$

введенного уравнения не являются рядами от $x$ и $y$, но обладают своеобразной ассоциативностью. Такие квадратные уравнения в [37] были названы двузначными формальными группами, определенными квадратом модуля формальной группы.

В [57] было дано аксиоматическое определение двузначной формальной группы над кольцом $A$, задаваемой уравнением

$$
Z^{2}-\Theta_{1}(x, y) Z+\Theta_{2}(x, y)=0,
$$

где $\Theta_{k}(x, y) \in A[[x, y]], k=1,2$. 
Обозначим через $\Lambda \subset \Omega_{U}$ подкольцо, порожденное коэффициентами рядов $\Theta_{k}(x, y) \in \Omega_{U}[[x, y]], k=1,2$, полученных описанной выше конструкцией в случае формальной группы геометрических кобордизмов. Соответствующая двузначная формальная группа над кольцом $\Lambda$ названа двузначной формальной группой в кобордизмах.

В цикле работ автора настоящей статьи было доказано, что двузначная формальная группа в кобордизмах является универсальной среди двузначных групп, удовлетворяющих условию $\Theta_{2}(x, x)=0$.

Детальное изложение теории двузначных формальных групп и ее топологических приложений дано в [58]. Главным алгебраическим результатом этой теории является полная классификация двузначных формальных групп. Опишем топологический результат, получивший важные приложения в теории $S p^{*}(\cdot)$ кобордизмов стабильно симплектических многообразий, задаваемой спектром Тома $M S p=\{M S p(n)\}$. Кольцо скаляров этой теории $-\Omega_{S p}$.

В последние годы активно изучаются симплектические кобордизмы, построенные на основе симплектических многообразий в смысле симплектической геометрии [59]. В связи с этим появилось предложение, чтобы не было путаницы, называть теорию $S p^{*}(\cdot)$ теорией кватернионных кобордизмов, но мы будем придерживаться устоявшейся терминологии.

В работе С.П. Новикова [11] методом классической спектральной последовательности Адамса было показано, что

$$
\Omega_{S p}\left[\frac{1}{2}\right] \equiv \mathbb{Z}\left[\frac{1}{2}\right]\left[b_{n}, n=1, \ldots\right], \quad \operatorname{deg} b_{n}=-4 n .
$$

Полное вычисление кольца $\Omega_{S p}$, включая структуру кольца $\Omega_{S p} /(2$-кручение), остается одной из самых известных нерешенных задач алгебраической топологии.

Каноническое отображение спектров $j: M S p \rightarrow M U$, соответствующее стандартному вложению групп $S p(n) \subset U(2 n)$, задает кольцевой гомоморфизм $j_{*}: \Omega_{S p} \rightarrow \Omega_{U}$ такой, что Ker $j_{*}\left[\frac{1}{2}\right]=0$, и эпиморфизм $\mathscr{A}_{U}$-модулей $j^{*}: \mathscr{A}_{U} \rightarrow$ $U^{*}(M S p)$. В [58] построена теория функтора

$$
L^{*}(X)=\operatorname{Hom}_{\mathscr{A}_{U}}\left(U^{*}(M S p), U^{*}(X)\right)
$$

и вычислены кольца $L^{*}(X)$ для важных случаев, когда $X$ - произведение комплексных проективных пространств. Например, доказано, что

$$
L^{4 *}(\mathbb{C} P(\infty) \times \mathbb{C} P(\infty))=L[[x, y, Z]] /\left\langle Z^{2}-\Theta_{1}(x, y) Z+\Theta_{2}(x, y)\right\rangle,
$$

где $L=L^{*}(\mathrm{pt})$. Кольца $L^{*}(X)$ играют ключевую роль в вычислении колец $S p^{*}(X)$ методом спектральной последовательности Адамса-Новикова. Отметим, что если пространство $X$ не имеет 2 -кручения в гомологиях, то кольцо $L^{*}(X)$ является минимальным аддитивно прямым слагаемым в кольце $U^{*}(X)$, содержащим образ кольца $S p^{*}(X)$. Особый интерес, естественно, представляет кольцо $L=L^{*}(\mathrm{pt}) \subset \Omega_{U}$, которое можно отождествить с кольцом комплексных 
кобордизмов стабильно комплексных многообразий $M^{m}$, обладающих накрытиями $\widehat{M}^{m} \rightarrow M^{m}$ со стабильно симплектической структурой, т. е. многообразие $\widehat{M}^{m}$ допускает вложение $\widehat{M}^{m} \subset \mathbb{R}^{m+4 n}$, нормальное расслоение которого имеет структурную группу $S p(n)$.

Пусть $\Lambda_{1}$ - подкольцо в $\Lambda\left[\frac{1}{2}\right]$, порожденное коэффициентами рядов $\frac{1}{2} \Theta_{1}(x, y)$ и $\Theta_{2}(x, y)$. В [58] доказано, что $L=\Lambda_{1} \cap \Omega_{U}$ и

$$
\Lambda_{1}=\mathbb{Z}\left[c_{n}, n \geqslant 1\right], \quad \operatorname{deg} c_{n}=-4 n .
$$

В. В. Вершинин, используя это вычисление кольца $L$, получил важный результат о произведении элементов порядка 2 в кольце $\Omega_{S p}$ (см. [60]) методом спектральной последовательности Адамса-Новикова.

Любая двузначная формальная группа над кольцом $A$, определяемая уравнением

$$
Z^{2}-\Theta_{1}(x, y) Z+\Theta_{2}(x, y)=0
$$

где $\Theta_{2}(x, x)=0$, задает совокупность рядов $\left\{\phi_{k}(x), k \in \mathbb{Z}\right\}$, где $\phi_{k}(x)=\phi_{-k}(x)$, $\phi_{0}(x)=0, \phi_{1}(x)=x$ и

$$
\phi_{k}(x)=\Theta_{1}\left(x, \phi_{k-1}(x)\right)-\phi_{k-2}(x) .
$$

Ряды $\phi_{k}(x)=k^{2} x+\left(x^{2}\right)$ представляют собой степенную систему типа 2. Эта система не является системой, задаваемой формальной группой над кольцом $A$ (cм. [37]), так как формальная группа $B^{-1}(B(x)+B(y))$ не лежит в кольце $A[[x, y]]$, где $B(x) \in A \otimes \mathbb{Q}$ - логарифм степенной системы $\left\{\phi_{k}(x)\right\}$ (см. выше).

Двузначная формальная группа над кольцом $L$, задаваемая квадратом модуля формальной группы геометрических кобордизмов $f(u, v)$ над кольцом $\Omega_{U}$, является универсальной среди двузначных групп таких, что $\Theta_{2}(x, x)=0$ (см. [58]). Обозначим через $L(2)$ подкольцо в $L$, порожденное коэффициентами соответствующей степенной системы $\left\{\phi_{k}(x)\right\}$ типа 2. Таким образом, мы получаем реализацию над кольцом $L(2) \subset L \subset \Omega_{U}$ универсальной степенной системы типа 2, отличную от ее реализации степенной системой $\left\{\left[k^{2}\right](u)\right\}$ над кольцом $\Omega(2) \subset \Omega_{U}$. Следовательно, существует изоморфизм колец $\Omega(2) \rightarrow L(2)$. Этот изоморфизм удваивает размерность классов кобордизмов.

Описанная система $\left\{\phi_{k}(x)\right\}$ типа 2 имеет важные топологические применения и впервые возникла в неявном виде в [23] при описании неподвижных точек действия 2-групп обобщенных кватернионов на стабильно комплексных многообразиях.

Конструкция степенной системы $\phi_{k}(x)$ имеет естественное обобщение. Пусть $F(u, v)$ - формальная группа над кольцом $R$ без кручения и $g_{F}(u)$ - ее логарифм. Рассмотрим полный набор $\left(\xi_{0}=1, \ldots, \xi_{m-1}\right)$ корней $m$-й степени из 1. Положим $B_{m}^{-1}(-y)=\prod_{j=0}^{m-1} g_{F}^{-1}\left(\xi_{j} \sqrt[m]{y}\right), x=\prod_{j=0}^{m-1} g_{F}^{-1}\left(\xi_{j} g_{F}(u)\right)$. Тогда $B_{m}(x)=(-1)^{m-1} g_{F}(u)^{m}$, и мы получаем формальную степенную систему

$$
F_{k}^{(m)}(x)=B_{m}^{-1}\left(k^{m} B_{m}(x)\right)=\prod_{j=0}^{m-1} g_{F}^{-1}\left(k \xi_{j} g_{F}(u)\right)
$$


типа $s=m$. Коэффициенты ряда $F_{k}^{(m)}(x)$ заведомо лежат в кольце $R$ для формальных групп $F(u, v)$ с комплексным умножением на $\xi_{j}$ (возведением в степень $\left.\xi_{j}\right)$. Эта конструкция проходит для формальной группы геометрических кобордизмов над кольцом $\Omega_{U} \otimes \mathbb{Z}_{p}$ и $m=p-1$, где $\mathbb{Z}_{p}$ - кольцо целых $p$-адических чисел. Как и в случае $m=2$, можно рассмотреть также $m$-значную формальную группу, задаваемую алгебраическим уравнением степени $m$, решения которого имеют вид $F_{k}(x, y)=B_{m}^{-1}\left(\left(\sqrt[m]{B_{m}(x)}+\right.\right.$ $\left.\left.\varepsilon^{k} \sqrt[m]{B_{m}(y)}\right)^{m}\right)$, где $k=0, \ldots, m-1$ и $\varepsilon^{m}=1$. Топологические конструкции и приложения $m$-значных групп можно найти в [61], обзор по их теории - в [62].

\section{2. Квантовые группы и операторные дубли}

Пусть $k$ - некоторое коммутативное кольцо. Скажем, что алгебры $A$ и $M$ над $k$ образуют тройку Милнора $(A, M ; \varphi)$, если $A$ - алгебра Хопфа, а $M$ - некоторый $A$-модуль с милноровским действием $\varphi: A \otimes M \rightarrow M$, т.е. $a\left(m_{1} \cdot m_{2}\right)=\sum\left(a^{\prime} m_{1}\right)\left(a^{\prime \prime} m_{2}\right)$, где $\Delta a=\sum a^{\prime} \otimes a^{\prime \prime}-$ диагональ в $A$ и $a m=$ $\varphi(a \otimes m)$.

В 1990-е годы, когда большое внимание было привлечено к квантовым группам (см. [18], [19]), С. П. Новиков, исследуя связь конструкции В. Г. Дринфельда с алгеброй $\mathscr{A}_{U}$, ввел в [16] понятие операторного дубля $M_{\varphi} A$ тройки Милнора $(A, M ; \varphi)$.

Операторный дубль $M_{\varphi} A$ представляет собой алгебру над $k$ такую, что:

- алгебра $M_{\varphi} A$ содержит алгебры $A$ и $M$ как подалгебры;

- $k$-линейное отображение $M \otimes_{k} A \rightarrow M_{\varphi} A: m \otimes a \rightarrow m \cdot a$ является изоморфизмом;

- выполняется правило коммутации $a \cdot m=\sum a^{\prime}(m) \cdot a^{\prime \prime}$.

Далее, когда ясно, о каком действии $\varphi$ алгебры $A$ на $M$ идет речь, операторный дубль $M_{\varphi} A$ обозначается короче: $M A$. Ясно, что операторный дубль $\Omega_{U} S$ для канонического представления $S$ на $\Omega_{U}$ совпадает с алгеброй $\mathscr{A}_{U}$ (см. [16]).

Пусть $A$ - некоторая алгебра Хопфа над $k$ и $A^{*}=\operatorname{Hom}_{k}(A, k)$ - двойственная ей алгебра Хопфа. Обозначим через $r, l$ и ad милноровские действия алгебры Хопфа $A$ на $A^{*}$, задаваемые формулами

$$
\begin{gathered}
\langle r(x) \sigma, y\rangle=\langle\sigma, y x\rangle, \quad\langle l(x) \sigma, y\rangle=\langle\sigma, \gamma(x) y\rangle, \\
\langle a d(x) \sigma, y\rangle=\left\langle\sigma, \sum \gamma\left(x^{\prime}\right) y x^{\prime \prime}\right\rangle
\end{gathered}
$$

где $x, y \in A, \sigma \in A^{*}, \gamma$ - антиавтоморфизм алгебры Хопфа $A,\langle\cdot, \cdot\rangle$ - каноническое спаривание $A^{*} \otimes A \rightarrow k$. Таким образом, для алгебры Хопфа $A$ определены операторные дубли

$$
A_{r}^{*} A, \quad A_{l}^{*} A \quad \text { и } \quad A_{a d}^{*} A .
$$

В случае, когда алгебра Хопфа $A$ является кокоммутативной, операторный дубль $A_{a d}^{*} A$ совпадает (см. [16]) с квантовой группой алгебры Хопфа $A$ в конструкции В. Г. Дринфельда. 
В [16] отмечается, что операторные дубли естественно возникают как алгебры дифференциальных операторов на группах Ли или алгебры разностных операторов на дискретных группах. В этих случаях $M$ - алгебра функций на группе со стандартной структурой алгебры Хопфа, а $X=M^{*}$ - двойственная алгебра Хопфа. Аналогичная конструкция под именем "гейзенбергов дубль" одновременно и независимо появилась в работе М. А. Семенова-Тян-Шанского (см. [20]).

Как следует из [63] (см. разд. 17), алгебра всех операций в теории комплексных кобордизмов изоморфна подалгебре в алгебре дифференциальных операторов на группе $\operatorname{Diff}_{1}(\mathbb{Z})$.

Развитие теории и приложений операторных дублей было дано в [17]. В этой работе, в частности, показано, что квантовая группа $S_{a d}^{*} S$ алгебры ЛандвебераНовикова $S$ изоморфна подалгебре Хопфа алгебры когомологических операций в теории удвоенных комплексных кобордизмов $D U^{*}(\cdot)$. Теория $D U^{*}(\cdot)$ представима спектром $M U \wedge M U$. Она строится на основе гладких многообразий, в нормальном расслоении которых фиксировано расщепление в сумму двух комплексных расслоений. Для удвоенной теории комплексных кобордизмов $D U^{*}(\cdot)$ имеются преобразования (см. разд. 4)

$$
\mu_{D U}^{l}: U^{*}(\cdot) \rightarrow D U^{*}(\cdot) \quad \text { и } \quad \mu_{D U}^{r}: U^{*}(\cdot) \rightarrow D U^{*}(\cdot),
$$

задающие вложения

$$
\mu_{D U}^{l}: \Omega_{U} \rightarrow \Omega_{D U} \quad \text { и } \quad \mu_{D U}^{r}: \Omega_{U} \rightarrow \Omega_{D U} .
$$

Положим $\mu_{D U}^{l}\left(\Omega_{U}\right)=\Omega_{U, l}$ и $\mu_{D U}^{r}\left(\Omega_{U}\right)=\Omega_{U, r}$. На кольце когомологий точки $D U^{*}(\mathrm{pt})=\Omega_{D U}=\pi_{*}(M U \wedge M U)$ теории $D U^{*}(\cdot)$ существуют операторы, задающие расщепляющие изоморфизмы

$$
\Omega_{U, l} \otimes S_{r}^{*} \stackrel{\simeq}{\rightarrow} \Omega_{D U} \stackrel{\simeq}{\longleftarrow} S_{l}^{*} \otimes \Omega_{U, r} .
$$

Описание этих изоморфизмов в терминах формальной группы геометрических кобордизмов см. ниже.

Левая $u_{l}$ и правая $u_{r}$ ориентации универсального расслоения $\xi \rightarrow \mathbb{C} P^{\infty}$ определяют две формальные группы над кольцом $\Omega_{D U}$ :

$$
\begin{array}{ll}
f^{l}(x, y)=x+y+\sum \alpha_{i, j}^{l} x^{i} y^{j}, & \alpha_{i, j}^{l} \in \Omega_{U, l}, \\
f^{r}(x, y)=x+y+\sum \alpha_{i, j}^{r} x^{i} y^{j}, & \alpha_{i, j}^{r} \in \Omega_{U, r} .
\end{array}
$$

Как отмечалось выше, любые две ориентации связаны рядом, следовательно, существует ряд $\phi(x)=x+\sum b_{k}^{r} x^{k+1}, b_{k}^{r} \in \Omega_{D U}$, такой, что $u_{r}=\phi\left(u_{l}\right)$ и

$$
\phi\left(f^{l}(x, y)\right)=f^{r}(\phi(x), \phi(y)),
$$

т. е. ряд $\phi(x)$ задает сильный изоморфизм групп $f^{l}(x, y)$ с $f^{r}(x, y)$. В [17] доказано, что относительно вложений $\mu_{D U}^{l}$ и $\mu_{D U}^{r}$ имеют место изоморфизмы:

$$
\Omega_{U, l}\left[b_{n}^{r}, n=1,2, \ldots\right] \simeq \Omega_{D U} \simeq \Omega_{U, r}\left[b_{n}^{l}, n=1,2, \ldots\right],
$$

где $b_{n}^{l}$ - коэффициенты ряда $\phi^{-1}(x)$. 
Пусть $\left(h^{*}(\cdot), u_{1}, u_{2}\right)$ - некоторая $\mathbb{C}$-ориентированная теория когомологий с двумя ориентациями. Тогда имеются мультипликативное преобразование

$$
\mu_{h}: U^{*}(\cdot) \rightarrow h^{*}(\cdot) ; \quad \mu_{h} u=u_{1}
$$

формальная группа

$$
f_{h}(x, y)=x+y+\sum a_{i, j}^{h} x^{i} y^{j}, \quad a_{i, j}^{h} \in \Omega_{h},
$$

и ряд

$$
\phi(x)=x+\sum b_{n}^{h} x^{n+1}, \quad b_{n}^{h} \in \Omega_{h},
$$

такой, что $u_{2}=\phi\left(u_{1}\right)$.

Из предыдущих конструкций следует, что преобразование $\mu_{h}$ продолжается до мультипликативного преобразования

$$
D \mu_{h}: D U^{*}(X) \rightarrow h^{*}(X)
$$

которое для $X=\mathrm{pt}$ дает гоморфизм

$$
D \mu_{h}: \Omega_{U, l}\left[b_{n}^{r}, n=1,2, \ldots\right] \rightarrow \Omega_{h}
$$

такой, что

$$
D \mu_{h}\left(\alpha_{i, j}^{l}\right)=a_{i, j}^{h} \quad \text { и } \quad D \mu_{h}\left(b_{n}^{r}\right)=b_{n}^{h} .
$$

Универсальные свойства теории $\left(D U^{*}(\cdot), u^{l}, u^{r}\right)$ можно описать в другой форме, эквивалентной предыдущей.

Пусть $\left(h^{*}(\cdot), u\right)$ - некоторая $\mathbb{C}$-ориентированная теория когомологий и $F(u, v)$ - некоторая формальная группа над $\Omega_{h}$, сильно изоморфная формальной группе $f_{h}(x, y)$, т. е. существует ряд $\phi(x)=x+\sum b_{n}^{h} x^{n+1}, b_{n}^{h} \in \Omega_{h}$, такой, что

$$
\phi\left(f_{h}(x, y)\right)=F(\phi(x), \phi(y)) .
$$

Тогда преобразование $\mu_{h}$ продолжается до мультипликативного преобразования $D \mu_{h}$, действующего на кольце скаляров по той же формуле.

Рассмотрим теперь алгебру когомологических операций в теории $D U^{*}(\cdot)$. В этой алгебре имеется подалгебра Хопфа $D S=S^{l} \otimes S^{r}$ - тензорное произведение алгебр Хопфа, где $S^{l}$ и $S^{r}$ - алгебры операций Ландвебера-Новикова, построенные по ориентациям $u_{l}$ и $u_{r}$ соответственно.

Аналогично изоморфизму для алгебры $\mathscr{A}_{U}$, имеет место изоморфизм $\mathscr{A}_{D U}=$ $\Omega_{D U}\left(S^{l} \otimes S^{r}\right)$. В [17] доказано, что подкольцо $B=\mathbb{Z}\left[b_{n}^{r}, n=1,2, \ldots\right] \subset \Omega_{D U} \simeq$ $\Omega_{U, l}\left[b_{n}^{r}, n=1,2, \ldots\right]$ замкнуто относительно действия алгебры $D S$ и, следовательно, является модулем Милнора над алгеброй Хопфа $S \otimes S$.

Следовательно, алгебра $\mathscr{A}_{D U}$ содержит в качестве подалгебры операторный дубль $B(S \otimes S)$.

Пусть $S^{*}$ - алгебра Хопфа, двойственная алгебре Хопфа $S$. Тогда существует изоморфизм $S^{*} \rightarrow B$, который вместе с диагональным гомоморфизмом $\Delta: S \rightarrow S \otimes S$ задает вложение квантового дубля $D(S)$ в операторный дубль $B(S \otimes S) \subset \mathscr{A}_{D U}$. 
В [54] дана следующая алгебраическая характеризация кольца $\Omega_{U}$ и универсальной формальной группы над ним.

Рассмотрим категорию колец $\mathscr{K}$, являющихся модулями над левым операторным дублем $S_{l}^{*} S$ алгебры Хопфа $S$ и модулями Милнора над $S$.

Пусть $K \in \mathscr{K}$. Элемент $v \in K$ называется геометрическим, если $s v=0$ при $s \neq s_{n}$ и $s_{n} v=v^{n+1}$.

Рассмотрим кольцо $S^{*}[[u, v]]$, где $u$ и $v$, по определению, геометрические элементы. Тогда $S^{*}[[u, v]] \in \mathscr{K}$ и существует ряд $\mathscr{F}(u, v) \in S^{*}[[u, v]]$, который однозначно определяется условием, что $w=\mathscr{F}(u, v)$ является геометрическим элементом. Более того, из единственности геометрического элемента $\mathscr{F}(u, v)$ такого, что $\mathscr{F}(u, 0)=u$, а $u$ и $v$ геометрические, вытекает, что ряд $\mathscr{F}(u, v)$ задает формальную группу над кольцом $S^{*}$. Аналогично доказывается, что существует ряд $g(x)=x+\sum b_{i} x^{i+1}, b_{i} \in S^{*}$, который однозначно определяется условием, что $s g(x)=0, s \in S_{2 q}, q>0$, для любого геометрического элемента $x \in S^{*}[[u, v]]$, и, следовательно,

$$
\mathscr{F}(u, v)=g^{-1}(g(u)+g(v))
$$

Обозначим через $\Omega$ подкольцо в $S^{*}$, порожденное коэффициентами ряда $\mathscr{F}(u, v)$.

Таким образом, ряд $\mathscr{F}(u, v)$ над кольцом $\Omega$ задает закон сложения, относительно которого множество геометрических элементов кольца $S^{*}[[u, v]]$ является группой. Этот закон является универсальным для функтора из категории $\mathscr{K}$ в категорию множеств, сопоставляющего кольцу $K \in \mathscr{K}$ множество его геометрических элементов.

Используя, что $U^{*}(X) \otimes_{\Omega_{U}} S^{*} \in \mathscr{K}$ для любого пространства $X$, мы получаем, что формальная группа $\mathscr{F}(u, v)$ совпадает с формальной группой геометрических кобордизмов. Это дает алгебраическую характеризацию кольца $\Omega_{U}$ и формальной группы геометрических кобордизмов в терминах алгебры Ландвебера-Новикова.

\section{3. Задачи о действиях групп}

Вернемся теперь в 1968 год. С. П. Новиков в [23] развил и уточнил результаты своей работы [12] по известной задаче вычисления характеристических чисел modulo $p$ многообразия $M^{n}$, несущего действие циклической группы $\mathbb{Z} / p$ (см. [64]). Для действий с изолированными неподвижными точками были получены явные формулы. K решению задач в этом направлении успешно подключились ученики С.П. Новикова (см. [65]-[70]). Важные результаты о кобордизмах многообразий с действием окружности были получены в [45], [46], [71]-[74]. В [23] была поставлена задача: извлечь из формул, полученных методами теории кобордизмов, знаменитые результаты Атья-Ботта о голоморфных действиях группы $\mathbb{Z}_{p}$ с изолированными неподвижными точками.

С.П. Новиков обсудил формулы Атья-Ботта с Д.К. Фаддеевым, который предложил записать их с помощью оператора теоретико-числового следа и провел показательные вычисления. Вскоре эта задача была полностью решена (см. [37]) для классических родов Хирцебруха. Впоследствии Т. Е. Панов в [75] 
решил эту задачу для эллиптического рода Виттена-Ошанина (см. разд. 15), используя оператор следа для расширения поля модулярных форм.

Результаты работы [23] опирались на открытую С.П.Новиковым связь родов Хирцебруха с логарифмом формальной группы геометрических кобордизмов.

Рассмотрим ряд $Q(t)=1+\sum a_{k} t^{k}$ и положим

$$
L_{Q}=L_{Q}\left(\sigma_{1}, \ldots, \sigma_{n}\right)=\prod_{q=1}^{n} Q\left(t_{q}\right),
$$

где $\sigma_{k}=\sigma_{k}\left(t_{1}, \ldots, t_{n}\right)-k$-я элементарная симметрическая функция. Классический род Хириебруха $L_{Q}\left(M^{2 n}\right)$ задается формулой

$$
L_{Q}\left(M^{2 n}\right)=\left(L_{Q}\left(c_{1}, \ldots, c_{n}\right),\left\langle M^{2 n}\right\rangle\right),
$$

где $c_{k}=c_{r}\left(\tau\left(M^{2 n}\right)\right)$ - классы Чженя в обычных когомологиях стабильного комплексного касательного расслоения и $\left\langle M^{2 n}\right\rangle$ - фундаментальный цикл многообразия в гомологиях. Впервые аналог этой формулы появился в [76] для ориентированных многообразий $M^{4 n}$ и их классов Понтрягина. В [23] доказано следующее утверждение. Пусть $f(t)=t / Q(t)$ и $f^{-1}(t)-$ ряд такой, что $f^{-1}(f(t))=t$. Тогда

$$
f^{-1}(t)=t+\sum_{n \geqslant 1} L_{Q}\left(\left[\mathbb{C} P^{n}\right]\right) \frac{t^{n+1}}{n+1} .
$$

Род Хирцебруха $L_{Q}$ задает кольцевой гомоморизм кольца $\Omega_{U}$.

Верно и следующее утверждение: каждый кольцевой гомоморфизм кольца $\Omega_{U}$ в кольцо без кручения можно однозначно представить с помощью рода Хирцебруха $L_{Q}$ для некоторого ряда $Q$. Развитие этого результата см. в разд. 14.

Используя изоморфизм $\Omega_{U} \otimes \mathbb{Q} \simeq \mathbb{Q}\left[\left[\mathbb{C} P^{n}\right], n=1,2, \ldots\right]$, мы получаем по формуле С.П. Новикова эффективное решение следующей задачи: найти ряд $Q(t)$ по значениям $L_{Q}\left(\left[\mathbb{C} P^{n}\right]\right)$. В современной литературе термины "род Хирцебруха" и "кольцевой гомоморфизм кольца $\Omega_{U}$ в кольцо без кручения" считаются синонимами.

Опишем, следуя [77], недавние результаты о действиях окружности на многообразиях.

Рассмотрим $2 n$-мерное многообразие $M$ с гладким действием $\theta$ окружности $S^{1}$ на нем. Говорят, что $M$ обладает структурой нормального комплексного $S^{1}$-многообразия $\left(M, \theta, c_{\nu}\right)$, если заданы линейное действие $S^{1}$ на $\mathbb{C}^{k}$ и эквивариантное вложение $i: M \rightarrow D^{2 k} \subset \mathbb{C}^{k}, i(M) \cap \partial D^{2 k}=\varnothing$, вместе с фиксированной эквивариантной комплексной структурой $c_{\nu}$ на нормальном расслоении $\nu(i)$. Используя стандартное диагональное действие группы $S^{1}$ на сфеpe $S^{2 m+1} \subset \mathbb{C}^{m+1}$ и действие $S^{1}$ на $D^{2 k} \subset \mathbb{C}^{k}$, введем гладкие многообразия $\mathscr{M}_{m}=S^{2 m+1} \times_{S^{1}} M$ и $W_{m}=S^{2 m+1} \times_{S^{1}} D^{2 k}$. Вложение $i$ продолжается до вложения $i^{\prime}: \mathscr{M}_{m} \rightarrow W_{m}$, так что комплексная структура $c_{\nu}$ продолжается до комплексной структуры $c^{\prime}$ на нормальном расслоении $\nu\left(i^{\prime}\right)$.

Мы получили $\mathbb{C}$-ориентированное отображение $i^{\prime}$ многообразия $\mathscr{M}_{m}$ размерности $2(m+n)$ в $2(m+k)$-мерное многообразие $W_{m}$ с границей $\partial W_{m}$ такое, что 
$\mathcal{M}_{m} \cap \partial W_{m}=\varnothing$ и $W_{m} / \partial W_{m}$ является пространством Тома $T \zeta$ комплексного $k$-мерного расслоения $\zeta=\left(S^{2 m+1} \times_{S^{1}} \mathbb{C}^{k}\right) \rightarrow \mathbb{C} P^{m}$.

Таким образом, однозначно определен класс комплексных кобордизмов $\Phi_{m}\left(M, \theta, c_{\nu}\right) \in U^{-2 n}\left(\mathbb{C} P^{m}\right)$ такой, что $i_{!}^{\prime}(1)=T_{U} \Phi_{m}\left(M, \theta, c_{\nu}\right)$, где $1 \in \Omega_{U}^{0} \subset$ $U^{0}\left(\mathcal{M}_{m}\right)$ и $i_{!}^{\prime}: U^{0}\left(\mathcal{M}_{m}\right) \rightarrow U^{2(k-n)}\left(W_{m} / \partial W_{m}\right)$ - гомоморфизм Гизина вложения $i^{\prime}$. Здесь $T_{U}: \widetilde{U}^{-2 n}\left(\mathbb{C} P^{m}\right) \rightarrow \widetilde{U}^{2(k-n)}(T \zeta)-$ изоморфизм Тома.

Проверяется, что $i_{m}^{*} \Phi_{m+1}=\Phi_{m}$, где $r_{m}: \mathbb{C} P^{m} \rightarrow \mathbb{C} P^{m+1}-$ стандартное вложение. Обратная последовательность $\left\{\Phi_{m}\left(M, \theta, c_{\nu}\right): m=0,1, \ldots\right\}$ задает элемент $\Phi\left(M, \theta, c_{\nu}\right) \in U^{-2 n}\left(\mathbb{C} P^{\infty}\right)$, который называется универсальным $S^{1}$-эквивариантным родом структуры $\left(M, \theta, c_{\nu}\right)$. Непосредственно из конструкции следует, что ряд $\Phi\left(M, \theta, c_{\nu}\right)$ по переменной $u$ при $u=0$ принимает значение $[M]$.

Скажем, что $M$ обладает структурой тангенииального стабилъно комплексного $S^{1}$-многообразия $\left(M, \theta, c_{\nu}\right)$, если заданы некоторое комплексное $l$-мерное расслоение $\zeta \rightarrow M$ и изоморфизм $c_{\tau}: \tau(M) \oplus \mathbb{C}^{l-n} \rightarrow \zeta$, где $\tau(M)$ - вещественное касательное расслоение, такие, что композиция

$$
\rho(t): \zeta \stackrel{c_{\tau}^{-1}}{\longrightarrow} \tau(M) \oplus \mathbb{C}^{l-n} \stackrel{d \theta(t)+I}{\longrightarrow} \tau(M) \oplus \mathbb{C}^{l-n} \stackrel{c_{\tau}}{\longrightarrow} \zeta
$$

является комплексным преобразованием для каждого $t \in S^{1}$, где $d \theta(t)$ - дифференциал преобразования $\theta(t)$. Таким образом, определено представление $\rho: S^{1} \rightarrow \operatorname{Hom}_{\mathbb{C}}(\zeta, \zeta)$. С точностью до естественной эквивалентности структура $\left(M, \theta, c_{\tau}\right)$ определяет структуру $\left(M, \theta, c_{\nu}\right)$, и поэтому можно ввести $\Phi\left(M, \theta, c_{\tau}\right)$ как $\Phi\left(M, \theta, c_{\nu}\right)$ для соответствующей структуры $c_{\nu}$. Важно иметь в виду, что обратное, вообще говоря, неверно, т. е. $c_{\nu}$ не всегда определяет эквивариантную структуру $c_{\tau}$.

Пусть $x \in M-$ изолированная неподвижная точка действия $\theta$. Тогда представление $\rho_{x}: S^{1} \rightarrow G L(l, \mathbb{C})$ разлагает слой в сумму $\mathbb{C}^{n} \oplus \mathbb{C}^{l-n}$, где $\rho_{x}(t)$ действует на $\mathbb{C}^{n}$ как $\rho_{x, 1} \oplus \cdots \oplus \rho_{x, n}$ и тривиально на $\mathbb{C}^{l-n}$. Здесь $\rho_{x, j}$ - одномерное комплексное представление веса $\omega_{x, j}$, т. е. $\rho_{x, j}(t) v=t^{\omega_{x, j}} v$, где $\omega_{x, j} \neq 0$. Таким образом, каждой изолированной неподвижной точке $x$ сопоставляется набор весов $\omega_{x}=\left(\omega_{x, 1}, \ldots, \omega_{x, n}\right) \in(\mathbb{Z} \backslash\{0\})^{n}$. Более того, изоморфизм

$$
c_{x, \tau}: \tau_{x} \oplus \mathbb{C}^{l-n} \rightarrow \zeta_{x}
$$

индуцирует ориентацию в касательном пространстве $\tau_{x}$, с помощью которой вводится функция знака $\sigma(x)$ на множестве $X$ изолированных неподвижных точек $x \in M$ такая, что $\sigma(x)=1$, если композиция линейных отображений

$$
\tau_{x}(M) \stackrel{I \oplus 0}{\rightarrow} \tau_{x}(M) \oplus \mathbb{C}^{l-n} \stackrel{c_{\tau, x}}{\rightarrow} \xi_{x} \cong \mathbb{C}^{n} \oplus \mathbb{C}^{l-n} \rightarrow \mathbb{C}^{n}
$$

сохраняет ориентацию, и $\sigma(x)=-1$, если не сохраняет, где $\pi$ - проекция на первое слагаемое с канонической ориентацией в $\mathbb{C}^{n}$.

Имеет место ТЕОРЕМА ЛОКАЛИЗАЦИИ эквивариантного рода:

Пусть множество $X$ неподвижных точек $S^{1}$-многообразия $\left(M, \theta, c_{\tau}\right)$ конечно. Тогда в группе $U^{-2 n}\left(\mathbb{C} P^{\infty}\right) \subset \Omega_{U}[[u]]$ верна формула

$$
\Phi\left(M, \theta, c_{\tau}\right)=\sum_{x \in X} \sigma(x) \prod_{i=1}^{n} \frac{1}{\left[\omega_{x, i}\right](u)}=[M]+\mathscr{R}(M),
$$


где $[\cdot](u)$ - степенная система формальной группы геометрических кобордизмов (см. разд. 8), $[M]$ - класс кобордизмов многообразия $M$. Ряды $\mathscr{R}(M)$ описаны в [77], их коэффициенты представляют собой классы кобордизмов пространств расслоений со слоем многообразие $M$ и базой, кобордантной нулю.

Для почти комплексного $S^{1}$-многообразия $M$ (т.е. $l=n$ ) мы имеем $\sigma(x) \equiv 1$ и формула для $\Phi\left(M, \theta, c_{\tau}\right)$ переходит в формулу Кричевера [45].

Каждое слагаемое в формуле для $\Phi\left(M, \theta, c_{\tau}\right)$ имеет полюс порядка $n$ при $u=0$. Утверждение о том, что эквивариантный род задается рядом по $u$, т. е. не имеет особенностей при $u=0$, накладывает сильное условие на возможные наборы весов и знаков $\left\{\omega_{x} \in(\mathbb{Z} \backslash\{0\})^{n}, \sigma(x), x \in X\right\}$ структуры $\left(M, \theta, c_{\tau}\right)$.

Задача описания условий на возможные наборы весов в неподвижных точках действия конечной группы $\mathbb{Z} / p$ была поставлена в [12], где они названы условиями Коннера-Флойда. Эти условия изучались в [12], [65]-[67].

В работах Т. Е. Панова [78], [79] получены явные формулы для важных алгебро-топологических инвариантов многообразий $M^{2 n}$ со структурой $\left(M, \theta, c_{\tau}\right)$ (детали см. в [80]), в том числе формула

$$
\left(c_{n}\left(\tau\left(M^{2 n}\right)\right),\left\langle M^{2 n}\right\rangle\right)=\sum_{x \in X} \sigma(x)
$$

В случае, когда структура $c_{\tau}$ на $M^{2 n}$ эквивалентна почти комплексной структуре, имеем $\sum_{x \in X} \sigma(x)=\chi\left(M^{2 n}\right)$, где $\chi\left(M^{2 n}\right)$ - эйлерова характеристика многообразия $M^{2 n}$. Согласно теореме Э. Томаса [81], $2 n$-мерное вещественное ориентируемое расслоение $\xi$ допускает комплексную структуру тогда и только тогда, когда оно допускает стабильно комплексную структуру $\zeta$ такую, что $c_{n}(\zeta)=$ $e(\xi)$, где $e(\xi)$ - эйлеров класс расслоения $\xi$. В частности, $\left(e\left(\tau\left(M^{2 n}\right)\right),\left\langle M^{2 n}\right\rangle\right)=$ $\chi\left(M^{2 n}\right)$. Таким образом, условие $\sigma(x)=1$ для всех неподвижных точек действия $\left(M, \theta, c_{\tau}\right)$ является необходимым для существования $T^{k}$-инвариантной почти комплексной структуры.

В работе В. М. Бухштабера, Т. Е. Панова и Н. Рэя [82] для действий тора $T^{k}$, $k \geqslant 1$, были рассмотрены $T^{k}$-многообразия $\left(M, \theta, c_{\tau}\right)$ и в случае, когда действие $\theta$ имеет изолированные неподвижные точки, получена формула локализации универсального $T^{k}$-рода $\Phi\left(M, \theta, c_{\tau}\right) \in \Omega_{U}\left[\left[u_{1}, \ldots, u_{k}\right]\right]$ в терминах знаков $\sigma(x)$ и весов $\omega_{x}$ неподвижных точек $x \in M$. Эта формула нашла важные приложения ввиду того, что такие действия играют ключевую роль в разных направлениях современных исследований. Приведем наиболее известные классы действий тора $T^{k}$ с изолированными неподвижными точками.

В последние 30 лет под влиянием задач, пришедших из физики, активно развивается торическая геометрия - раздел алгебраической геометрии. Центральным объектом здесь является торическое многообразие. Первые топологические аналоги таких многообразий были введены М. Дэвисом и Т. Янушкевичем в [83] и впоследствии получили название квазиторических. Более подробно о квазиторических многообразиях см. [80]. 
Приведем, следуя книге [80], конструкцию квазиторического многообразия $M(P, \Lambda)$, которая отличается от конструкции из [83]. Она является топологическим аналогом алгебро-геометрической конструкции В. В. Батырева неособых проективных торических многообразий (см. [84]).

Выпуклый $n$-мерный многогранник $P \subset \mathbb{R}^{n}$ называется простым, если в каждой его вершине сходится в точности $n$ гиперграней.

Пусть

$$
P=\left\{x \in \mathbb{R}^{n}:\left\langle a_{i}, x\right\rangle+b_{i} \geqslant 0,1 \leqslant i \leqslant m\right\},
$$

где $a_{i} \in \mathbb{R}^{n}$ и $b_{i} \in \mathbb{R}$. Предполагается, что ни одно из $m$ неравенств нельзя удалить.

Образуем $(m \times n)$-матрицу $A_{P}$, строки которой - векторы $a_{i}$, записанные в стандартном базисе. Положим $b=\left(b_{1}, \ldots, b_{m}\right) \in \mathbb{R}^{m}$. Образом отображения

$$
L_{P}: \mathbb{R}^{n} \rightarrow \mathbb{R}^{m}: L_{P}(x)=A_{P} x+b
$$

является пересечение $n$-мерной плоскости $\left\{y \in \mathbb{R}^{m}: y=A_{P} x+b\right\}$ с положительным конусом

$$
\mathbb{R}_{\geqslant}^{m}=\left\{y \in \mathbb{R}^{m}: y_{i} \geqslant 0, i=1, \ldots, m\right\}
$$

Каноническое действие стандартного тора $\mathbb{T}^{m}$ на $\mathbb{C}^{m}$ задает проекцию

$$
\rho: \mathbb{C}^{m} \rightarrow \mathbb{R}_{\geqslant}^{m}: \rho(z)=\left(\left|z_{1}\right|^{2}, \ldots,\left|z_{m}\right|^{2}\right)
$$

Вводится гладкое многообразие $\mathscr{Z}_{P}$ с каноническим действием тора $\mathbb{T}^{m}$ такое, что диаграмма

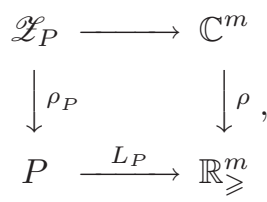

коммутативна.

Пусть $\left\{F_{1}, \ldots, F_{m}\right\}$ - множество гиперграней простого многогранника $P^{n}$. Целочисленная $(n \times m)$-матрица $\Lambda$ задает характеристическое отображение

$$
\ell:\left\{F_{1}, \ldots, F_{m}\right\} \rightarrow \mathbb{Z}^{n},
$$

если ее столбцы $\lambda_{j_{1}}, \ldots, \lambda_{j_{n}}$ образуют базис в $\mathbb{Z}^{n}$ для любой вершины $v=$ $F_{j_{1}} \cap \cdots \cap F_{j_{n}}$. Матрица $\Lambda$ задает эпиморфизм $\ell: \mathbb{T}^{m} \rightarrow \mathbb{T}^{n}$. Группа $K(\Lambda)=\operatorname{ker} \ell$ ранга $(m-n)$ действует свободно на $\mathscr{Z}_{P}$.

Пространство орбит $M=\mathscr{Z}_{P} / K(\Lambda)$ является $2 n$-мерным гладким многообразием с действием $n$-мерного тора $T^{n}=\mathbb{T}^{m} / K(\Lambda)$. Обозначим это действие через $\alpha$, оно удовлетворяет условиям из [83]:

1) $\alpha$ локально изоморфно стандартному действию $\mathbb{T}^{n}$ на $\mathbb{C}^{n}$;

2 ) существует проекция $\pi: M \rightarrow P$ такая, что $\pi^{-1}(x)$ является орбитой действия $\alpha$ для любого $x \in P$.

Многообразие $M=M(P, \Lambda)$ называется квазиторическим с комбинаторными данными $\left(A_{P}, \Lambda, b\right)$. 
В алгебраической геометрии неособое проективное торическое многообразие $M$ определяется (см. [84]) простым многогранником

$$
P=\left\{x \in \mathbb{R}^{n}:\left\langle a_{i}, x\right\rangle+b_{i} \geqslant 0, i=1, \ldots, m\right\},
$$

вершины которого лежат в решетке $\mathbb{Z}^{n}$, и наборы векторов $a_{i_{1}}, \ldots, a_{i_{n}}$ для каждой вершины $v=F_{i_{1}} \cap \cdots \cap F_{i_{n}}$ образуют базис решетки $\mathbb{Z}^{n}$.

В этом случае определено характеристическое отображение $\ell:\left\{F_{1}, \ldots, F_{m}\right\} \rightarrow$ $\mathbb{Z}^{n}$ при помощи матрицы $A_{P}^{\top}$, которая задает эпиморфизм $\ell: \mathbb{T}^{m} \rightarrow \mathbb{T}^{n}$ и его комплексификацию $\ell_{\mathbb{C}}:\left(\mathbb{C}^{*}\right)^{m} \rightarrow\left(\mathbb{C}^{*}\right)^{n}$, где $\mathbb{C}^{*}=\mathbb{C} \backslash\{0\}$.

Комбинаторные данные $\left(A_{P}, b, \Lambda\right)$ задают каноническую $T^{n}$-инвариантную тангенциальную стабильно комплексную структуру на многообразии $M(P, \Lambda)$ и позволяют для каждой неподвижной точки $x$ вычислить знак $\sigma(x)$ (см. ниже), причем, если $T^{n}$-инвариантная структура является почти комплексной, то $\sigma(x)=1$ для всех неподвижных точек.

Рассмотрим проекцию $\pi: M(P, \Lambda) \rightarrow P$. Множество гиперграней $\left\{F_{1}, \ldots, F_{m}\right\}$ многогранника $P$ определяет множество взаимно транверсальных подмногообразий $M_{j}=\pi^{-1}\left(F_{j}\right), j=1, \ldots, m$, коразмерности 2 в $M(P, \Lambda)$. Пусть $v=F_{j_{1}} \cap$ $\cdots \cap F_{j_{n}}$ - вершина многогранника $P$. Тогда $x=\pi^{-1}(v)=M_{j_{1}} \cap \cdots \cap M_{j_{n}}$ - неподвижная точка $T^{n}$-действия $\alpha$ на $M(P, \Lambda)$. Имеем взаимно однозначное соответствие между множеством вершин $\left\{v_{1}, \ldots, v_{N}\right\}$ и множеством неподвижных точек $\left\{x_{1}, \ldots, x_{N}\right\}$ действия $\alpha$. Касательное пространство $\tau_{x}$ к $M=M(P, \Lambda)$ в неподвижной точке $x$ как $2 n$-мерное вещественное пространство разлагается в сумму $\tau_{x}(M(P, \Lambda))=\left.\left.\nu_{j_{1}}\right|_{x} \oplus \cdots \oplus \nu_{j_{n}}\right|_{x}$, где $\nu_{j} \rightarrow M_{j}-$ нормальное расслоение к подмногообразию $M_{j}$ в $M$.

В терминах комбинаторных данных задается структура комплексного одномерного расслоения в $\nu_{j}$ для каждого $j=1, \ldots, m$.

Пусть $x=M_{j_{1}} \cap \cdots \cap M_{j_{n}}-$ неподвижная точка. Тогда $\sigma(x)=1$, если ориентация пространства $\tau_{x}(M)$, задаваемая стабильно комплексной структурой в $M$, совпадает с ориентацией расслоения $\left.\left.\nu_{j_{1}}\right|_{x} \oplus \cdots \oplus \nu_{j_{n}}\right|_{x}$, определенного ориентациями расслоений $\nu_{j k}, k=1, \ldots, m$, и $\sigma(x)=-1$ в противном случае. Имеет место формула

$$
\sigma(x)=\operatorname{sign}\left(\operatorname{det}\left(\lambda_{j_{1}}, \ldots, \lambda_{j_{n}}\right) \operatorname{det}\left(a_{j_{1}}, \ldots, a_{j_{n}}\right)\right) .
$$

Понятие знака $\sigma(x)$ неподвижной точки $x$ действия тора $\mathbb{T}^{n}$ на квазиторическом многообразии $M^{2 n}$ сыграло важную роль в известной задаче о представителях классов стабильно-комплексных кобордизмов.

Согласно теореме Милнора (см. [28]) в каждом классе $\left[M^{2 n}\right] \in \Omega_{U}^{-2 n}$ содержится неособое алгебраическое многообразие. Метод доказательства этой теоремы использует многообразия Милнора (см. разд. 6 после формулы (19)) и приводит к несвязным многообразиям. В 1958 г. в докладе на Международном математическом конгрессе в Эдинбурге Ф. Хирцебрух (см. [96]) поставил задачу: описать классы $\left[M^{2 n}\right] \in \Omega_{U}^{-2 n}$, содержащие неприводимое (т. е. связное) неособое алгебраическое многообразие. Эта задача до сих пор остается нерешенной.

В [85] доказано, что для каждого $n>1$ в группе $\Omega_{U}^{-2 n}$ существует базис, составленный из классов стабильно комплексных кобордизмов квазиторических 
многообразий. Стандартная операция связной суммы $M_{1}^{2 n} \# M_{2}^{2 n}$ квазиторических многообразий вдоль окрестностей неподвижных точек $x_{k} \in M_{k}^{2 n}, k=1,2$, дает многообразие $M^{2 n}$ с действием тора $T^{n}$. В [86] доказано, что это многообразие имеет структуру квазиторического многообразия, согласованную с квазиторическими структурами на $M_{1}^{2 n}$ и $M_{2}^{2 n}$ тогда и только тогда, когда $\sigma\left(x_{1}\right) \sigma\left(x_{2}\right)=-1$.

В [86] введена новая операция $M_{1}^{2 n} \diamond M_{2}^{2 n}$ соединения квазиторических многообразий - "бриллиантовая сумма" такая, что в группе $\Omega_{U}^{-2 n}$ имеет место формула

$$
\left[M_{1}^{2 n} \diamond M_{2}^{2 n}\right]=\left[M_{1}^{2 n}\right]+\left[M_{2}^{2 n}\right] .
$$

Операция $\diamond$ позволяет соединять квазиторические многообразия вдоль неподвижных точек одного знака. В частности, определено квазиторическое многообразие $M_{1}^{2 n} \diamond M_{2}^{2 n}$ в случае торических многообразий $M_{1}$ и $M_{2}$.

В качестве следствия сформулированного выше результата из [85] в [86] получено:

Каждый класс стабилъно комплексных кобордизмов $\left[M^{2 n}\right] \in \Omega_{U}^{-2 n}, n>1$, содержит квазиторическое многообразие.

В этой формулировке нельзя убрать приставку “квази". Препятствием является, например, тот факт, что любое торическое многообразие имеет род Тодда 1.

Отметим решение еще одной известной задачи, использующее понятие знака $\sigma(x)$ неподвижной точки $x \in M^{2 n}$.

А. А. Кустарев [87] показал, что если $\sigma(x)=1$ для любой неподвижной точки, то каноническая $T^{n}$-инвариантная стабильно комплексная структура на $M(P, \Lambda)$ эквивалентна $T^{n}$-инвариантной почти комплексной структуре. Таким образом, для комбинаторных данных $\left(A_{P}, b, \Lambda\right)$ существует $T^{n}$-инвариантная почти комплексная структура на квазиторическом многообразии $M(P, \Lambda)$ тогда и только тогда, когда $\sigma(x)=1$ (см. формулу выше) для любой неподвижной точки $x$.

Другой важный класс $\mathbb{T}^{k}$-многообразий $\left(M, \theta, c_{\tau}\right)$ с изолированными неподвижными точками, когда знаки и веса неподвижных точек вычисляются эффективно, дает классическая теория однородных пространств $G / H$ компактных групп Ли. Здесь $\theta$ - действие максимального тора $T^{k}$ группы $G$ на однородном пространстве $G / H$.

В. М. Бухштабером и С. Терзич в [88] получены формулы для $\Phi\left(G / H, \theta, c_{\tau}\right)$ в терминах теории групп Ли и в качестве следствия вычислены классы комплексных кобордизмов комплексных многообразий флагов и многообразий Грассмана.

\section{4. Роды Хирцебруха}

Пусть $A$ - некоторое коммутативное кольцо без кручения с единицей 1 и $f(t)=t+\sum_{k \geqslant 1} f_{k} t^{k+1}$, где $f_{k} \in A \otimes \mathbb{Q}$. Положим

$$
Q(t)=\frac{t}{f(t)}
$$

Роды Хирцебруха $L_{Q}$ (см. разд. 13 ) далее будем обозначать $L_{f}$. 
Род $L_{f}: \Omega_{U} \rightarrow A \otimes \mathbb{Q}$ называется $A$-иелочисленным, если $L_{f}\left(M^{2 n}\right) \in A$ для любого стабильно комплексного многообразия $M^{2 n}$.

Благодаря универсальности формальной группы геометрических кобордизмов над $\Omega_{U}$, множество формальных групп над $A$ взаимно однозначно соответствует множеству кольцевых гомоморфизмов $\Omega_{U} \rightarrow A$. При этом формальная группа $F$ классифицируется родом Хирцебруха $L_{f}: \Omega_{U} \rightarrow A$, где $f(t)$ - экспонента этой группы. Следовательно, множество всех $A$-целочисленных родов $L_{f}$ совпадает с множеством кольцевых гомоморфизмов $L_{f}: \Omega_{U} \rightarrow A$ и множеством всех формальных групп над $A$. Обратим внимание, что решение задачи описания всех $\mathbb{Z}$-целочисленных родов Хирцебруха, таким образом, следует из работы Т. Хонда [89], которая опирается на фундаментальные результаты теории чисел. В этой работе получена классификация формальных групп над $\mathbb{Z}$.

Тождественному отображению $\Omega_{U} \rightarrow \Omega_{U}$ соответствует род Хирцебруха $L_{f}$, где $f(t)$ - экспонента формальной группы геометрических кобордизмов. Этот род детально изучен в [38] под именем универсального рода Хирцебруха.

Напомним, что для любого $n$-мерного комплексного расслоения $\zeta \rightarrow X$ определены функториальные классы Тома $u(\zeta) \in U^{2 n}(T(\zeta))$ и $u_{H}(\zeta) \in H^{2 n}(T(\zeta))$ такие, что $u_{H}(\zeta)=\mu_{H} u(\zeta)$, где $\mu_{H}: U^{*}(\cdot) \rightarrow H^{*}(\cdot ; \mathbb{Z})$ - каноническое мультипликативное преобразование (см. разд. 3 и 4$)$.

Введем характеристический класс $\mathscr{P}(\zeta) \in H^{0}\left(X ; \Omega_{U}(\mathbb{Z})\right)$ формулой

$$
T_{u_{H}(\zeta)} \mathscr{P}(\zeta)=\operatorname{ch}_{U} u(\zeta),
$$

где $T_{u_{H}(\zeta)}$ - изоморфизм Тома в когомологиях.

Непосредственно из определения следует, что $\mathscr{P}(n)=1$, где $n$ - тривиальное расслоение над $X$, и для любых комплексных расслоений $\zeta_{1}$ и $\zeta_{2}$ над $X$ имеет место формула $\mathscr{P}\left(\zeta_{1} \oplus \zeta_{2}\right)=\mathscr{P}\left(\zeta_{1}\right) \mathscr{P}\left(\zeta_{2}\right)$. Таким образом, определен экспоненииальный гомоморфизм

$$
\mathscr{P}: K^{0}(X) \rightarrow\left(1+\widetilde{H}^{0}\left(X, \Omega_{U}(\mathbb{Z})\right)\right) .
$$

Этот гомоморфизм полностью определяется тем, что для универсального расслоения $\xi \rightarrow \mathbb{C} P^{\infty}$ имеет место формула $t \mathscr{P}(\xi)=f(t)$, где $t \in H^{2}\left(\mathbb{C} P^{\infty} ; \mathbb{Z}\right)$ и $f(t)$ - экспонента формальной группы геометрических кобордизмов.

Общим характеристическим классом Тодда $\mathscr{T}(\zeta)$ комплексного расслоения $\zeta$ называется класс $\mathscr{P}(-\zeta)=\mathscr{P}(\zeta)^{-1}$ (см. [38]).

Выбор названия объясняется тем, что класс $\mathscr{T}(\zeta)$ возникает в $U^{*}$-аналоге общей теоремы Римана-Роха-Хирцебруха-Гротендика.

Рассмотрим, как и выше, некоторое $\mathbb{C}$-ориентированное отображение многообразий

$$
f: M^{m} \rightarrow W^{l}, \quad \partial M=\varnothing, \quad f(M) \cap \partial W=\varnothing,
$$

с комплексным нормальным расслоением $\nu$ вложения $M^{m} \subset W^{l} \times \mathbb{R}^{q+m}$.

Мультипликативные преобразования $\mu_{H}: U^{*}(\cdot) \rightarrow H^{*}(\cdot, \mathbb{Z})$ и $\operatorname{ch}_{U}: U^{*}(\cdot) \rightarrow$ $H^{*}\left(\cdot, \Omega_{U}(\mathbb{Z})\right)$ задают две ориентации $u_{H}(\nu)=\mu_{H} u(\nu)$ и $\operatorname{ch}_{U} u(\nu)$ расслоения $\nu$. Следовательно, для $U^{*}$-гомоморфизма Гизина

$$
f_{!}: U^{k}\left(M^{m}\right) \rightarrow U^{k+l-m}\left(W^{l}, \partial W^{l}\right) .
$$


Мы получаем формулу (см. разд. 3)

$$
\operatorname{ch}_{U} f_{!}(x)=\left(f_{!}\right)_{H}(\mathscr{T}(-\nu) x),
$$

где $\mathscr{T}(-\nu)=\rho\left(\operatorname{ch}_{U} u(\nu), u_{H}(\nu)\right)$ - класс, различающий ориентации.

В случае, когда многообразия $M^{m}$ и $W^{l}$ ориентированы в обычных когомологиях, имеют место изоморфизмы двойственности Пуанкаре:

$$
\begin{aligned}
& D_{M}: H^{k}\left(M^{m} ; \mathbb{Z}\right) \rightarrow H_{m-k}\left(M^{m} ; \mathbb{Z}\right) \\
& D_{W}^{-1}: H_{m-k}\left(W^{l} ; \mathbb{Z}\right) \rightarrow H^{l+k-m}\left(W^{l} . \partial W^{l} ; \mathbb{Z}\right)
\end{aligned}
$$

и $\left(f_{!}\right)_{H}=\mathscr{D}_{W}^{-1} f_{*} \mathscr{D}_{M}$.

В случае, когда многообразия $M^{m}$ и $W^{l}$ ориентированы в $U^{*}$-теории, т. е. когда касательные расслоения $\tau\left(M^{m}\right)$ и $\tau\left(W^{l}\right)$ являются стабильно комплексными, мы получаем формулу

$$
\operatorname{ch}_{U} f_{!}(x)=\mathscr{D}_{W}^{-1} f_{*} \mathscr{D}_{M}\left(\frac{\mathscr{T}(\tau(M))}{\mathscr{T}(\tau(W))} x\right) .
$$

В случае, когда $M^{2 n}$ - стабильно комплексное многообразие, а $W$ - точка, мы получаем: $f_{!}(1)=\left[M^{2 n}\right]$ и

$$
\left[M^{2 n}\right]=\operatorname{ch}_{U}\left[M^{2 n}\right]=\left(\mathscr{T}(\tau(M)),\left\langle M^{2 n}\right\rangle\right) .
$$

Рассмотрим кольцевой гомоморфизм $\mathrm{Td}: \Omega_{U}(\mathbb{Z}) \rightarrow \mathbb{Q}\left[\beta^{-1}, \beta\right]$, ограничение которого на $\Omega_{U} \subset \Omega_{U}(\mathbb{Z})$ задается формулой $\operatorname{Td}\left[M^{2 n}\right]=\operatorname{Td}\left(M^{2 n}\right) \beta^{-n}$, где $\operatorname{Td}\left(M^{2 n}\right)$ - классический род Тодда стабильно комплексного многообразия. Напомним, что $\mathrm{Td}=L_{Q}$, где $Q(t)=\frac{t}{1-e^{-\beta t}}$.

Обозначим также $\mathrm{Td}: H^{*}\left(\cdot ; \Omega_{U}(\mathbb{Z})\right) \rightarrow H^{*}(\cdot ; \mathbb{Q})$ преобразование, определенное гомоморфизмом колец коэффициентов. Имеет место соотношение:

$$
\operatorname{Td~ch}_{U}=\operatorname{ch}_{K} \mu_{K}
$$

где $\mu_{K}: U^{*}(\cdot) \rightarrow K^{*}(\cdot)$ - преобразование Римана-Роха-Коннера-Флойда (см. разд. 4).

Для любого стабильно комплексного многообразия $M^{2 n}$ из приведенных выше результатов вытекает соотношение

$$
\operatorname{Tdch}_{U} f_{!}(x)=\operatorname{ch}_{K} \mu_{K} f_{!} x
$$

и, следовательно, соотношение

$$
\operatorname{ch}_{K}\left(f_{!}\right)_{K} \mu_{K} x=\left(\mu_{K} \mathscr{T}(\tau(M)) \operatorname{ch}_{K} \mu_{K} x,\langle M\rangle\right) .
$$

Гомоморфизм $\mathrm{Td}: \Omega_{U} \rightarrow \mathbb{Z}\left[\beta^{-1}, \beta\right]$ задает в кольце $\mathbb{Z}\left[\beta^{-1}, \beta\right]$ структуру $\Omega_{U^{-}}$ модуля, относительно которой имеет место известный изоморфизм КоннераФлойда

$$
U^{*}(X) \otimes_{\Omega_{U}} \mathbb{Z}\left[\beta^{-1}, \beta\right] \rightarrow K^{*}(X) .
$$


Из этого результата и предыдущего соотношения вытекает классическая формула Римана-Роха-Хирцебруха-Гротендика в комплексной $K$-теории:

$$
\operatorname{ch}_{K}\left(f_{!}\right)_{K} \zeta=\left(\mathscr{T}_{K}(\tau(M)) \operatorname{ch}_{K} \zeta,\langle M\rangle\right),
$$

где $\zeta \in K^{0}(M)$ и $\mathscr{T}_{K}(\cdot)$ - характеристический класс Тодда в $K$-теории.

В разд. 13 описан универсальный $S^{1}$-эквивариантный род $\Phi\left(M, \theta, c_{\nu}\right) \in$ $U^{*}\left(\mathbb{C} P^{\infty}\right)=\Omega_{U}[[u]]$ структуры $\left(M, \theta, c_{\nu}\right)$.

Любой род $L_{f}: \Omega_{U} \rightarrow A$ задает $S^{1}$-эквивариантный род $L_{f}\left(\Phi\left(M, \theta, c_{\nu}\right)\right)=$ $\Phi_{f}\left(M, \theta, c_{\nu}\right) \in A[[u]]$, который можно рассматривать как деформацию с параметром $u$ рода $L_{f}[M]$. По построению, деформация соответствует структуре $\left(M, \theta, c_{\nu}\right)$ действия окружности $S^{1}$ на многообразии $M$. Это приводит к следующему понятию.

Структура $\left(M, \theta, c_{\nu}\right)$ называется жесткой для рода $L_{f}$, если

$$
\Phi_{f}\left(M, \theta, c_{\nu}\right)(u)=\Phi_{f}\left(M, \theta, c_{\nu}\right)(0)=L_{f}[M] .
$$

На основе универсального $T^{k}$-эквивариантного рода $\Phi\left(M, \theta, c_{\nu}\right) \in U^{*}\left(B T^{k}\right)$ вводятся $T^{k}$-эквивариантные роды $\Phi_{f}\left(M, \theta, c_{\nu}\right) \in A\left[\left[u_{1}, \ldots, u_{k}\right]\right]$.

Аналогично вводятся универсальные $G$-эквивариантные роды $\Phi\left(M, \theta, c_{\nu}\right) \in$ $U^{*}(B G)$, где $G$ - компактная группа Ли, $B G$ - ее классифицирующее пространство и $\theta$ - действие группы $G$ на $M$ для некоторого $G$-эквивариантного вложения $M \subset \mathbb{R}^{N}$ с комплексным нормальным расслоением $\nu$.

Отметим, что в случае группы $G$ все результаты о жестких структурах для родов $L_{f}$ извлекаются из результатов о $T^{k}$-эквивариантных родах, где $T^{k} \subset G-$ максимальный тор группы $G$.

Результаты о жестких структурах для классических родов, привлекшие большое внимание к этой теме, были получены в [90] на основе теоремы Атья-Зингера. В [45], [74] был развит метод, основанный на использовании аналитических свойств "соотношений Коннера-Флойда", полученных из формул локализации эквивариантных родов в терминах формальных групп. В [45] получен ряд результатов о жесткости, в частности новое доказательство результатов Атья и Хирцебруха.

И. М. Кричевер в [46] ввел род $L_{f}$, получивший впоследствии его имя, где $f(t)=\frac{e^{\lambda / 2} t}{\Phi(t)}$ и $\Phi(t)-$ эллиптическая функция Бейкера-Ахиезера, играющая фундаментальную роль в теории интегрируемых систем.

В [46] доказано, что структура $\left(M, \theta, c_{\nu}\right)$ для рода Кричевера является жесткой, если $c_{1}\left(c_{\nu}\right)=0$.

Замечательная характеризация этого рода была получена в [91] и [92] в связи с задачей построения аналогов родов Хирцебруха для компактных особых алгебраических многообразий.

\section{5. Эллиптические формальные группы}

Опишем, следуя работе [93], приложение классических теорий эллиптических кривых и эллиптических функций к проблеме $A$-целочисленности родов Хирцебруха и проблеме построения родов Хирцебруха, жестких для определенных структур $\left(M, \theta, c_{\nu}\right)$. 
Общая модель Вейеритрасса эллиптической кривой в координатах $(X: Y$ : $Z) \in \mathbb{C} P^{2}$ задается уравнением

$$
Y^{2} Z+\mu_{1} X Y Z+\mu_{3} Y Z^{2}=X^{3}+\mu_{2} X^{2} Z+\mu_{4} X Z^{2}+\mu_{6} Z^{3},
$$

зависящим от вектора параметров $\mu=\left(\mu_{1}, \mu_{2}, \mu_{3}, \mu_{4}, \mu_{6}\right)$.

В карте $\{Y \neq 0\}$ с координатами $u=-X / Y$ и $s=-Z / Y$ это уравнение принимает вид

$$
s=u^{3}+\mu_{1} u s+\mu_{2} u^{2} s+\mu_{3} s^{2}+\mu_{4} u s^{2}+\mu_{6} s^{3},
$$

однородный относительно градуировки $\operatorname{deg} u=2, \operatorname{deg} s=6, \operatorname{deg} \mu_{i}=-2 i$. Здесь $u$ и $s$ - арифметические координаты Тейта (см. [94]). Они получили такое название, так как в окрестности точки $(0,0)$ координата $s$ задается рядом $s(u) \in \mathbb{Z}\left[\mu_{1}, \mu_{2}, \mu_{3}, \mu_{4}, \mu_{6}\right][[u]]$ и, таким образом, $u$ является униформизирующей координатой на этой кривой.

Геометрическая групповая структура на эллиптической кривой задается следующим условием: если точки $P_{1}, P_{2}, P_{3}$ лежат на пересечении некоторой прямой с данной кривой, то $P_{1}+P_{2}+P_{3}=0$. Фиксируем нуль геометрической групповой структуры в точке $O=(0: 1: 0)$. Тогда в полученной группе из соотношений $P_{1}+P_{2}+P_{3}=0$ и $P_{3}+\bar{P}_{3}+O=0$ вытекает $P_{1}+P_{2}=\bar{P}_{3}$.

Пусть $(u, s(u)),(v, s(v)),(w, s(w))$ и $(\bar{w}, s(\bar{w}))$ - координаты точек $P_{1}, P_{2}, P_{3}$ и $\bar{P}_{3}$ в карте $\{Y \neq 0\}$. Геометрическая групповая структура в координатах $(u, s(u))$ задает закон сложения формальной группы над кольцом $\mathbb{Z}\left[\mu_{1}, \mu_{2}, \mu_{3}\right.$, $\left.\mu_{4}, \mu_{6}\right]$ уравнением $\mathscr{F}_{\mathrm{El}}(u, v)=\bar{w}$. Эта формальная группа названна общей эллиптической формальной группой.

В [93] получен явный вид ряда $\mathscr{F}_{\mathrm{El}}(u, v) \in \mathbb{Z}\left[\mu_{1}, \mu_{2}, \mu_{3}, \mu_{4}, \mu_{6}\right][[u, v]]$ и дифференциальные уравнения на экспоненту общей эллиптической формальной группы.

Формальная группа $\mathscr{F}_{\mathrm{El}}(u, v)$ определяет $\mathbb{Z}\left[\mu_{1}, \mu_{2}, \mu_{3}, \mu_{4}, \mu_{6}\right]$-целочисленный род Хирцебруха, названный общим эллиптическим родом $L_{\mathrm{El}}$.

Формальная группа над кольцом $A$ называется эллиптической и обозначается $F_{\mathrm{El}}(u, v)$, если классифицирующий ее кольцевой гомоморфизм $\mathscr{A} \rightarrow A$ разлагается в композицию $\mathscr{A} \rightarrow \mathbb{Z}\left[\mu_{1}, \mu_{2}, \mu_{3}, \mu_{4}, \mu_{6}\right] \rightarrow A$.

Положим

$$
c_{2}=\mu_{1}^{2}+4 \mu_{2}, \quad c_{4}=\mu_{1} \mu_{3}+2 \mu_{4}, \quad c_{6}=\mu_{3}^{2}+4 \mu_{6}
$$

и

$$
g_{2}(\mu)=\frac{1}{12} c_{2}^{2}-2 c_{4}, \quad g_{3}(\mu)=\frac{1}{6} c_{2} c_{4}-\left(\frac{1}{6} c_{2}\right)^{3}-c_{6} .
$$

Имеем $\operatorname{deg} g_{i}=-4 i$. Экспонента формальной группы $\mathscr{F} \mathrm{El}(u, v)$ задается формулой

$$
f_{\mathrm{El}}(t)=-2 \frac{\wp(t)-\left(4 \mu_{2}+\mu_{1}^{2}\right) / 12}{\wp^{\prime}(t)-\mu_{1} \wp(t)+\mu_{1}\left(4 \mu_{2}+\mu_{1}^{2}\right) / 12-\mu_{3}},
$$

где $\wp(t)=\wp\left(t ; g_{2}(\mu), g_{3}(\mu)\right)$ - классическая ю-функция Вейерштрасса [95]. 
Как отмечено в разд. 14, в работе [46] И. М. Кричевер ввел род Хирцебруха $L_{f}$, где

$$
f(t)=f_{\mathrm{Kr}}(t)=\frac{\exp ((\lambda / 2) t)}{\Phi(t)},
$$

и доказал, что этот род обладает фундаментальным свойством жесткости на многообразиях с $S^{1}$-эквивариантной $S U$-структурой.

Пусть $A$ - кольцо без делителей нуля. В [93] доказано, что для того чтобы экспонента эллиптической формальной группы $F_{\mathrm{El}}(u, v)$ над $A$ задавала род Кричевера, необходимо и достаточно, чтобы в $A$ выполнялись соотношения

$$
\mu_{2} \mu_{3}-\mu_{1} \mu_{4}=0, \quad \mu_{3}^{2}+3 \mu_{6}=0, \quad \mu_{3}\left(\mu_{1} \mu_{3}+\mu_{4}\right)=0 .
$$

Приведем примеры законов сложения формальных групп, экспоненты которых задают эллиптические роды Кричевера.

Пусть $\mu=\left(0, \mu_{2}, 0, \mu_{4}, 0\right)$. Тогда $f_{\mathrm{El}}(t)$ удовлетворяет уравнению $\left(f^{\prime}\right)^{2}=$ $1-2 \delta f^{2}+\varepsilon f^{4}$, где $\delta=\mu_{2}, \varepsilon=\mu_{2}^{2}-4 \mu_{4}$. То есть $f_{\mathrm{El}}(t)-$ эллиптический синус $s n(t)$, и $F_{\mathrm{El}}(u, v) \in \mathbb{Z}\left[\mu_{2}, \mu_{4}\right][[u, v]]$ задает классический закон сложения

$$
F_{\mathrm{El}}(u, v)=\frac{u \rho(v)+v \rho(u)}{1-\varepsilon u^{2} v^{2}}, \quad \text { где } \quad \rho(u)^{2}=1-2 \delta u^{2}+\varepsilon u^{4} .
$$

В этом случае род Хирцебруха $L_{f}$ является эллиптическим родом, введенным Ошаниным в [39].

Пусть $\mu=\left(0,0, \mu_{3}, 0, \mu_{6}\right)$, где $\mu_{3}^{2}=-3 \mu_{6}$. Тогда

$$
F_{\mathrm{El}}(u, v)=\frac{u^{2} r(v)-v^{2} r(u)}{u r(v)^{2}-v r(u)^{2}}, \quad \text { где } \quad r(u)^{3}=1-3 \mu_{3} u^{3} .
$$

В этом случае род $L_{f}$ является целочисленным над кольцом $\mathbb{Z}\left[\mu_{3}, \mu_{6}\right] / \mu_{3}^{2}+3 \mu_{6}$ и соответствующий ему эквивариантный род является жестким на многообразиях с $S^{1}$-эквивариантной $S U$-структурой.

Пусть $\mu=\left(\mu_{1}, \mu_{2}, 0,0,0\right)$. Тогда

$$
F_{\mathrm{El}}(u, v)=\frac{u+v-\mu_{1} u v}{1+\mu_{2} u v}, \quad g(u)=\frac{1}{\alpha-\beta} \ln \frac{1-\beta u}{1-\alpha u}, \quad f(t)=\frac{e^{\alpha t}-e^{\beta t}}{\alpha e^{\alpha t}-\beta e^{\beta t}},
$$

где $\mu_{1}=\alpha+\beta, \mu_{2}=-\alpha \beta$. В этом случае род Хирцебруха $L_{f}$ является известным двупараметрическим родом Тодда $T_{\alpha, \beta}$. Род $T_{\alpha, \beta}$ переходит в род Тодда при $\mu_{2}=0$; в L-род (сигнатуру, см. разд. 16) при $\mu_{1}=0$; в эйлерову характеристику при $\mu_{1}^{2}=-4 \mu_{2}$.

\section{6. Общая проблема Милнора-Хирцебруха}

Пусть $\gamma \in \mathscr{A}_{U}$ - операция в теории комплексных кобордизмов и $L: \Omega_{U} \rightarrow A-$ некоторый род Хирцебруха. Элемент $L\left(\gamma\left[M^{2 n}\right]\right) \in A$ называется $(L, \gamma)$-характеристическим числом стабильно комплексного многообразия $M^{2 n}$. В случае, когда $L=L_{H}: \Omega_{U} \rightarrow \mathbb{Z}$ - аугментация, т. е. $L_{H}\left(\left[M^{2 n}\right]\right)=0$ при $n>0$ и $L_{H}([\mathrm{pt}])=1$, мы получаем, что если $\gamma-$ операция Ландвебера-Новикова $s \in$ 
$S_{2 k}$, то $L_{H}\left(s\left(M^{2 n}\right)\right)=0$, при $k \neq n$ и $L_{H}\left(s\left(M^{2 n}\right)\right)$ - классическое характеристическое число, отвечающее характеристическому классу комплексного нормального расслоения многообразия $M^{2 n}$.

На Математическом конгрессе в Эдинбурге, 1958 г., в докладе Хирцебруха (см. [96]) была сформулирована задача: найти все соотношения делимости на множествах характеристических чисел комплексных многообразий. Теория комплексных кобордизмов привела к задаче, получившей известность как проблема Милнора-Хирцебруха: найти все соотношения делимости на множествах характеристических чисел стабильно комплексных многообразий. Атья и Хирцебрух (см. [30]), опираясь на теорему Римана-Роха-Хирцебруха-Гротендика, выдвинули гипотезу, что решение проблемы Милнора-Хирцебруха дают условия целочисленности всех $(L, \gamma)$-чисел, где $L=\mathrm{Td}: \Omega_{U} \rightarrow \mathbb{Z}-$ род Тодда.

Эта гипотеза была независимо доказана Р. Стонгом [97] и А. Хаттори [98].

Для каждого ориентируемого $4 k$-мерного многообразия $M$ определяется сигнатура $\tau(M)$ как разность чисел положительных и отрицательных квадратов формы индекса пересечения на $H_{2 k}(M ; \mathbb{Z})$. Сигнатура является инвариантом класса ориентированных бордизмов многообразия $M$. Для рода Хирцебруха $L_{Q}=T_{\alpha,-\alpha}$, где $Q(t)=t / \operatorname{th} t$, имеет место формула $L_{Q}\left[M^{4 k}\right]=\tau\left(M^{4 k}\right)$. Здесь th $t$ - гиперболический тангенс.

Непосредственно из определения сигнатуры $\tau(M)$ следует, что род Хирцебpуха $L_{Q}\left[M^{4 k}\right]=\tau\left(M^{4 k}\right)$ является гомотопическим инвариантом многообразия $M$. Благодаря этому факту результаты о делимости сигнатуры нашли фундаментальные приложения в теории многообразий. Наиболее известна в этом направлении теорема В. А. Рохлина:

Пусть $M^{4}$ - ориентируемое гладкое многообразие такое, что $M^{4} \backslash(\mathrm{pt})$ параллелизуемое многообразие. Тогда $\tau\left(M^{4}\right)$ делится на 16.

Гладкое многообразие $M^{n}$ является $S U$-многообразием, или многообразием Калаби-Яо в современной терминологии, если существует вложение $M^{n} \subset$ $\mathbb{R}^{n+2 m}$, нормальное расслоение $\nu$ которого имеет структуру комплексного расслоения с $c_{1}(\nu)=0$. Здесь $c_{1}(\cdot)$ - первый класс Чженя в когомологиях.

Хорошо известна теорема С. Д. Ошанина (см. [99]):

Сигнатура $\tau(M)$ любого $(8 k+4)$-мерного $S U$-многообразия $M$ делится на 16.

В [99] для всех $k$ построены $(8 k+4)$-мерные $S U$-многообразия $M$ с $\tau(M)=16$.

Пусть $\xi \rightarrow X-$ некоторое комплексное расслоение. Тогда $\xi+\bar{\xi}$ имеет каноническую структуру симплектического расслоения и определены классы Понтрягина $P_{\omega}(\xi+\bar{\xi}) \in U^{4|\omega|}(X)$ и операции $p_{\omega} \in \mathscr{A}_{U}$ (см. [58]).

В [100] (см. также [101]) на основе теории эллиптических функций, с использованием эллиптического рода Ошанина-Виттена и операции Адамса-Новикова $\Psi_{U}^{4}$ доказано, что для любого $\left[M^{4 n}\right] \in \Omega_{U}$ имеет место соотношение:

$$
L\left[M^{4 n}\right]+\sum(-1)^{\|\omega\|} L\left(P_{\omega}\left[M^{4 n}\right]\right) \equiv 0 \quad \bmod 2^{\alpha(n)},
$$

где $\alpha(n)$ - число единиц в двоичном разложении числа $n$ и $L: \Omega_{U} \rightarrow \mathbb{Z}-$ род Хирцебруха, сопоставляющий многообразию его сигнатуру. 
Этот результат привел к следующей задаче (ОБщАЯ ПРОБЛЕМА МиЛноРАХиРцЕБРУХА).

Пусть даны набор операций $\gamma_{1}, \ldots, \gamma_{N}$ в теории комплексных кобордизмов и род Хирцебруха $L: \Omega_{U} \rightarrow A$. Найти соотношения на характеристические числа $L \gamma_{i}\left(\left[M^{2 n}\right]\right), i=1, \ldots, N$, которые имеют место для любого стабильно комплексного многообразия $M^{2 n}$.

Важные результаты по общей проблеме Милнора-Хирцебруха получены K. Е. Фельдманом в [102]. В частности, доказано соотношение

$$
L\left[M^{4 n}\right]+\sum(-1)^{k} L\left(\pi_{k}\left[M^{4 n}\right]\right) \equiv 0 \bmod 2^{\alpha(n)},
$$

где $L$ и $\alpha(n)$ - такие же, как выше, и $\pi_{k}$ - операция, отвечающая характеристическому классу $\pi_{k}\left(M^{4 n}\right)=(-1)^{k}\left(c f_{2 k}\left(\tau\left(M^{4 n}\right)\right)\right)+c f_{2 k}\left(\tau\left(M^{4 n}\right)\right)$, где $c f_{2 k}(\cdot)-$ характеристические классы Чженя-Коннера-Флойда.

Отметим также следующий результат работы [102]: Пусть $M^{2 n}$ - почти комплексное многообразие, касательное расслоение которого допускает линейное подрасслоение. Тогда эйлерова характеристика многообразия $M^{2 n}$ является четной.

В [103] развит подход, позволивший на основе результатов по общей проблеме Милнора-Хирцебруха получить новые доказательства и обобщения известных "the miraculous cancellation formula" Альвареса-Виттена [104] и классической формулы Пика [105].

\section{7. Алгебры Ли векторных полей и нильмногообразия.}

В работе В. М. Бухштабера и А. В. Шокурова [63] была установлена связь алгебры $\mathscr{A}_{U}$ с алгеброй операторов на группе $\operatorname{Diff}_{1}(k)$, где $k$ - некоторое коммутативное кольцо с единицей. Элементами этой группы являются ряды вида

$$
x=x(t)=t+\sum_{n \geqslant 1} x_{n} t^{n+1}, \quad x_{n} \in k .
$$

Обозначение $\operatorname{Diff}_{1}(k)$ объясняется тем, что когда $k=\mathbb{R}$ - поле вещественных чисел, эта группа является подгруппой группы формальных диффеоморфизмов прямой $\mathbb{R}^{1}$. В группе $\operatorname{Diff}_{1}(k)$ умножение $x \cdot y$ задается формулой $(x \cdot y)(t)=y(x(t))$, т. е. подстановкой ряда $x(t)$ в ряд $y(t)$.

Положим $\operatorname{Diff}_{1}(k) \simeq G^{\infty}(k)$. Обозначим через $G_{m}^{\infty}(k)$ нормальный делитель в $G^{\infty}(k)$, порожденный рядами $\left\{t+\sum_{n \geqslant m+1} x_{n} t^{n+1}\right\}$, и через $G^{m}(k)$ подмножество полиномов $t+\sum_{n=1}^{m} x_{n} t^{n+1}$ в $G^{\infty}(k)$. Каноническая проекция $\pi_{m}: G^{\infty}(k) \rightarrow$ $G^{\infty}(k) / G_{m}^{\infty}(k)$ представляет собой ретракцию $G^{\infty}(k) \rightarrow G^{m}(k)$. Отождествляя $G^{m}(k)$ с $k^{m}$, мы получаем в $k^{m}$ с координатами $\left(x_{1}, \ldots, x_{m}\right)$ структуру алгебраической группы, умножение в которой задается формулой

$$
(x \cdot y)(t)=\pi_{m}(y(x(t))) .
$$


Таким образом, действие группы $G^{m}(k)$ на себе левыми сдвигами задает линейное представление этой группы в линейном пространстве $k^{m}$ и, следовательно, задает представление группы $G^{m}(k)$ на кольце полиномов $P_{m}(k)=$ $k\left[x_{1}, \ldots, x_{m}\right]$. Переходя к обратному пределу по $m$, мы получаем действие группы $\operatorname{Diff}_{1}(k)=\lim _{\longleftarrow} G^{m}(k)$ на кольце полиномов $P(k)=k\left[x_{1}, x_{2}, \ldots\right]$.

Обозначим через $\mathscr{S}$ алгебру всех $\operatorname{Diff}_{1}(\mathbb{Z})$-инвариантных формальных дифференциальных операторов в $P=P(\mathbb{Z})$, т. е. формальных степенных рядов от $\frac{\partial}{\partial x_{1}}, \frac{\partial}{\partial x_{2}}, \ldots$ с коэффициентами в $\mathbb{Q}\left[x_{1}, x_{2}, \ldots\right]$, переводящих $P$ в $P$ и коммутирующих с линейными гомоморфизмами, задаваемыми элементами группы $\operatorname{Diff}_{1}(\mathbb{Z})$. В работе [63] показано, что имеет место изоморфизм алгебры Ландвебера-Новикова $S$ с алгеброй операторов $\mathscr{S}$, который превращает $P$ в $S$-модуль Милнора. При этом из теоремы Стонга-Хаттори о делимости чисел Чженя стабильно комплексных многообразий извлекается, что кольцо кобордизмов $\Omega_{U}$, как $S$-модуль, отождествляется с $\mathscr{S}$-подмодулем $\Omega$ в $P$, представляющим собой подкольцо в $P$, порожденное однородными (относительно градуировки $\left.\operatorname{deg} x_{n}=-2 n\right)$ полиномами, остающимися в $P$ при правом сдвиге на ряд $(1-\exp (-t)) \in \operatorname{Diff}_{1}(\mathbb{Q})$. Таким образом, в [63] был установлен изоморфизм алгебр $\mathscr{A}_{U} \simeq \Omega_{U} S \rightarrow \Omega \mathscr{S}$. Топологические приложения этого изоморфизма можно найти в работах [25], [63], [106].

Описанное выше действие группы $G^{m}(k)$ на $k^{m}$ задается нижнетреугольными матрицами. В случае $k=\mathbb{R}$ мы получаем структуру нильпотентной группы на $\mathbb{R}^{m}$. В группе $G^{m}(\mathbb{R})=\mathbb{R}^{m}$ имеется кокомпактная решетка $G^{m}(\mathbb{Z})=\mathbb{Z}^{m}$. Таким образом, для каждого $m \geqslant 1$ мы получаем нильмногообразие $M^{m}=$ $G^{m}(\mathbb{R}) / G^{m}(\mathbb{Z})$ - пространство Эйленберга-Маклейна $K\left(G^{m}(\mathbb{Z}), 1\right)$. Эти многообразия образуют башню главных расслоений со слоем окружность $S^{1}$ :

$$
\cdots \rightarrow M^{m+1} \rightarrow M^{m} \rightarrow \cdots \rightarrow M^{2} \rightarrow M^{1}
$$

Здесь $M^{1}=S^{1}$ и $M^{2}=T^{2}$ - абелевы группы, а все $M^{m}, m \geqslant 3$, уже не группы. Многообразия $M^{m}$ естественно назвать скрученными торами. В работе [106] явно описаны дифференциальные 2-формы $\omega_{m}$ на $M^{m}$, имеющие максимально возможный ранг. Эти формы $\omega_{m}$ для четных $m$ задают на $M^{m}$ симплектическую структуру.

Многообразия $M^{m}$ представляют собой реализацию симплектических неформальных многообразий, которые в работе И. К. Бабенко и И. А. Тайманова [107] привели к построению симплектических неформальных односвязных многообразий. В работе $\Phi$. Джонсона и Э. Риса [108] многообразия $G^{m}(\mathbb{C}) / G^{m}(\mathbb{Z}+i \mathbb{Z})$ были использованы как явные примеры комплексных некэлеровых многообразий.

Алгебраическая структура групп $G^{\infty}(k)$ для конечных полей $k$ изучается давно в связи с задачами теории групп и теории чисел. Группа $G^{\infty}(\mathbb{Z})$ привлекла к себе внимание относительно недавно. Ее алгебраическая структура изучалась в работах [109], [110]. Алгебраическим и топологическим результатам о группе $G^{\infty}(\mathbb{Z})$ посвящен обзор [111]. В [110] показано, что группа $G^{\infty}(\mathbb{Z})$ 
имеет всего 4 образующих и

$$
G^{\infty}(\mathbb{Z}) /\left[G^{\infty}(\mathbb{Z}), G^{\infty}(\mathbb{Z})\right]=\mathbb{Z}+\mathbb{Z}+\mathbb{Z} / 2+\mathbb{Z} / 2
$$

Этот результат получен как следствие теоремы:

$$
G^{m}(\mathbb{Z}) /\left[G^{m}(\mathbb{Z}), G^{m}(\mathbb{Z})\right]=\mathbb{Z}+\mathbb{Z}+\mathbb{Z} / 2+\mathbb{Z} / 2 .
$$

для всех $m \geqslant 6$.

В [63] подалгебра $\widehat{S} \subset S$, порожденная операциями $s_{n}$, была отождествлена с алгеброй Хопфа-Ли, порожденной векторными полями $d_{k}=x^{k+1} \frac{d}{d x}, k \geqslant 1$ на вещественной прямой с координатой $x$. Задача вычисления когомологий этой алгебры была поставлена И. М. Гельфандом в докладе на Международном Математическом конгрессе в Ницце, 1970 г. (см. [112]), и ее решение опубликовано Л. В. Гончаровой в [113], [114]. А именно, утверждается, что группа $H^{s, t}(\widehat{S} ; \mathbb{Q})$ равна $\mathbb{Q}$, если $t=3 s^{2} \pm s$, и является нулевой в остальных случаях. Числа $3 s^{2} \pm s / 2$ называются пентагонными числами Эйлера. Из свойств этих чисел следует, что в биградуированном кольце $H^{*, *}(\widehat{S} ; \mathbb{Q})$ умножение тривиально.

Вычисление групп $H^{*, *}(\widehat{S} ; \mathbb{Q})$ в [113], [114] технически сложное и трудно проверяемое. Имеется ряд публикаций, использующих различные, в том числе и самые современные методы гомологической алгебры, для доказательства этого важного результата. Поиски в этом направлении продолжаются.

На основе теоремы Л. Гончаровой в [63] получено вычисление когомологий $H^{*}(S ; \mathbb{Q})$ алгебры Ландвебера-Новикова $S$. Приложение этого результата см. в [63], [25].

Связь алгебры $\mathscr{A}_{U}$ с группой $\operatorname{Diff}_{1}(\mathbb{Z})$ открыла пути приложений в алгебраической топологии результатов общей теории нильпотентных групп Ли [116], теории нильмногообразий [117], а также результатов о группе $\operatorname{Diff}_{1}(\mathbb{Z})$ [111].

С другой стороны, эта связь привела к развитию исследований алгебраической топологии и дифференциальной геометрии многообразий $K\left(G_{m}(\mathbb{Z}), 1\right)$. Напомним, что изучение башен главных расслоений со слоем окружность хорошо известная тема алгебраической топологии.

\section{8. Алгебраические кобордизмы}

Успехи теории комплексных кобордизмов, естественно, стимулировали распространение ее идей и методов на произвольные алгебраические поля. Построению алгебраических кобордизмов посвящена монография В. Снэйта [118] (см. также [119]), в центре внимания которой конструкция, позволившая получить и важные алгебро-топологические результаты, в том числе гомотопическую реализацию изоморфизма Коннера-Флойда $U^{*}(X) \otimes_{\Omega_{U}} \mathbb{Z}\left[\beta^{-1}, \beta\right] \cong K^{*}(X)$ (см. [120]), использованного в [12]. Здесь $K^{*}(X)$ - комплексная $K$-теория и структура $\Omega_{U}$-модуля в кольце $\mathbb{Z}\left[\beta^{-1}, \beta\right]$ задается родом Тодда $\mathrm{Td}: \Omega_{U} \rightarrow$ $\mathbb{Z}\left[\beta^{-1}, \beta\right]: \operatorname{Td}\left(\left[M^{2 n}\right]\right)=\operatorname{Td}\left(M^{2 n}\right) \beta^{n}$ (см. разд. 4 и 14$)$. 
В работах В.В. Воеводского был развит подход, отличный от подхода В. Снэйта. Были введены алгебраические кобордизмы над произвольными алгебраическими полями, представленные так называемым $\mathbb{P}^{1}$-спектром. В случае полей $\mathbb{C}$ и $\mathbb{R}$ они совпадают с комплексными и неориентированными кобордизмами соответственно. Эта теория сыграла ключевую роль в доказательстве гипотезы Милнора [121]. Работы В. В. Воеводского привлекли большое внимание к алгебраическим кобордизмам. Позднее более геометрический вариант его теории вместе с соответствующей алгеброй Ландвебера-Новикова был предложен М. Левином и Ф. Морелем в [15]. В этой работе введено понятие ориентированной теории когомологий на категории гладких квазипроективных схем над полем $k$. В случае поля $k$ характеристики 0 с помощью описанного в [35] подхода к теории комплексных кобордизмов на этой категории строится универсальная ориентированная теория когомологий, названная теорией алгебраических кобордизмов. Устанавливается связь алгебраических кобордизмов схемы $X$ с ее классическим кольцом Чжоу и группой Гротендика алгебраических векторных расслоений над $X$. Эта теория получила известность и развитие в алгебраической геометрии (см. [122], [123]).

В работе А. Вишика [124] в теории Левина-Мореля введены новые, так называемые симметрические, операции. Эти операции тривиально действуют на классах кобордизмов, реализуемых подмногообразиями, и, таким образом, являются препятствием в известной задаче о реализации алгебраического цикла алгебраическим подмногообразием. Исследованы свойства симметрических операций, описана их связь с операциями Ландвебера-Новикова, даны приложения, в том числе в задаче о рациональности циклов.

\section{Список литературы}

[1] В. М. Бухштабер, А. С. Мищенко, С. П. Новиков, "Формальные группы и их роль в аппарате алгебраической топологии", УМH, 26:2(158) (1971), 131-154; англ. пер.: V. M. Bukhshtaber, A. S. Mishchenko, S. P. Novikov, "Formal groups and their role in the apparatus of algebraic topology", Russian Math. Surveys, 26:2 (1971), 63-90.

[2] С. П. Новиков, “Топология в ХХ веке: взгляд изнутри”, УМH, 59:5(359) (2004), 3-28; англ. пер.: S.P. Novikov, "Topology in the 20th century: a view from the inside", Russian Math. Surveys, 59:5 (2004), 803-829.

[3] С. П. Новиков, Предисловие к кн.: Кобордизмы в Советском Союзе 1967-1979, Топологическая библиотека, 4, ред. С. П. Новиков, И. А. Тайманов, М.-Ижевск, Институт компьютерных исследований, 2011, 9-13.

[4] Кобордизмы в Советском Союзе 1967-1979, Топологическая библиотека, 4, ред. С. П. Новиков, И. А. Тайманов, М.-Ижевск, Институт компьютерных исследований, 2011, 584 с.

[5] С. П. Новиков, Топология, изд. 2-е, дополн., Институт компьютерных исследований, М.-Ижевск, 2002, 336 с.; англ. изд.: S. P. Novikov, Topology, Encyclopaedia Math. Sci., 12, Springer, Berlin, 1996, 319 pp.

[6] H. Poincaré, "Analysis situs", J. Ecole Polytech. (2), 1 (1895), 1-121; А. Пуанкаре, "Analysis situs", Избранные труды, т. II, Наука, М., 1972, 457-807.

[7] M. F. Atiyah, "Bordism and cobordism", Proc. Cambridge Philos. Soc., 57:2 (1961), 200-208. 
[8] R. Thom, "Quelques propriétés globales de variétés différentiables", Comm. Math. Helv., 28 (1954), 17-86.

[9] J. Milnor, "On the cobordism ring $\Omega_{*}$ and a complex analogue. Part I", Amer. J. Math., 82:3 (1960), 505-521.

[10] С. П. Новиков, "О некоторых задачах топологии многообразий, связанных с теорией пространств Тома", Докл. АН СССР, 132:5 (1960), 1031-1034; англ. пер.: S.P. Novikov, "Some problems in the topology of manifolds connected with the theory of Thom spaces", Soviet Math. Dokl., 1 (1960), 717-720.

[11] С.П. Новиков, "Гомотопические свойства комплексов Тома", Матем. сб., 57(99):4 (1962), 407-442.

[12] С. П. Новиков, "Методы алгебраической топологии с точки зрения теории кобордизмов", Изв. АН СССР. Сер. матем., 31:4 (1967), 855-951; англ. пер.: S.P. Novikov, "The methods of algebraic topology from the viewpoint of cobordism theory", Math. USSR-Izv., 1:4 (1967), 827-913.

[13] T. Katsura, Yu. Shimizu, K. Ueno, "Complex cobordism ring and conformal field theory over $\mathbb{Z} "$, Math. Ann., 291:3 (1991), 551-571.

[14] T. Coates, A. Givental, "Quantum cobordisms and formal group laws", The unity of mathematics, Progr. Math., 244, Birkhäuser Boston, Boston, MA, 2006, 155-171.

[15] M. Levine, F. Morel, Algebraic cobordism, Springer Monogr. Math., Springer, Berlin, 2007, xii+244 pp.

[16] С. П. Новиков, "Различные удвоения алгебр Хопфа. Алгебры операторов на квантовых группах, комплексные кобордизмы”, УМH, 47:5(287) (1992), 189-190; англ. пер.: S. P. Novikov, "Various doublings of Hopf algebras. Operator algebras on quantum groups, complex cobordisms", Russ. Math. Surveys, 47:5 (1992), 198-199.

[17] V. M. Buchstaber, "Semigroups of maps into groups, operator doubles, and complex cobordisms", Topics in topology and mathematical physics, Amer. Math. Soc. Transl. Ser. 2, 170, Amer. Math. Soc., Providence, RI, 1995, 9-31.

[18] В.Г. Дринфельд, "Алгебры Хопфа и квантовое уравнение Янга-Бакстера", Докл. АН СССР, 283:5 (1985), 1060-1064; англ. пер.: V. G. Drinfel'd, "Hopf algebras and the quantum Yang-Baxter equation", Soviet Math. Dokl., 32 (1985), $256-258$.

[19] К. Кассель, Квантовые группы, ФАЗИС, М., 1999; пер. с англ.: С. Kassel, Quantum groups, Grad. Texts in Math., 155, Springer-Verlag, New York, 1995, xii+531 pp.

[20] М. А. Семенов-Тян-Шанский, "Группы Пуассона-Ли. Квантовый принцип двойственности и скрученный квантовый дубль", ТМФ, 93:2 (1992), 302-329; англ. пер.: M. A. Semenov-Tyan-Shanskii, "Poisson-Lie groups. The quantum duality principle and the twisted quantum double", Theoret. and Math. Phys., 93:2 (1992), 1292-1307.

[21] M. Demazure, "Motifs des variétés algébriques", Séminaire N. Bourbaki, 1969/70, Exp. № 365, 1971, 19-38.

[22] P.S. Landweber, "Cobordism operations and Hopf algebras", Trans. Amer. Math. Soc., 129 (1967), 94-110.

[23] С. П. Новиков, "Операторы Адамса и неподвижные точки”, Изв. АН СССР. Сер. матем., 32:6 (1968), 1245-1263; англ. пер.: S. P. Novikov, "Adams operators and fixed points", Math. USSR-Izv., 2:6 (1968), 1193-1211.

[24] В. М. Бухштабер, "Модули дифференциалов спектральной последовательности Атья-Хирцебруха. II", Матем. сб., 83(125):1(9) (1970), 61-76; англ. пер.: V. M. Buhštaber, "Modules of differentials of the Atiyah-Hirzebruch spectral sequence. II", Math. USSR-Sb., 12:1 (1970), 59-75.

[25] А.В. Шокуров, "О соотношениях между числами Чженя квазикомплексных многообразий”, Матем. заметки, 26:1 (1979), 137-148; англ. пер.: 
A. V. Shokurov, "Relations between the Chern numbers of quasicomplex manifolds", Math. Notes, 26:1 (1979), 560-566.

[26] D. C. Ravenel, Complex cobordism and stable homotopy groups of spheres, 2nd ed., AMS Chelsea Publishing, Providence, RI, 2004, xix+395 pp.

[27] H. Miller, Kervaire invariant one [after M.A. Hill, M. J. Hopkins, and D. C. Ravenel], Séminaire N. Bourbaki, 2010/2011, Exp. № 1029, 63ème année, 2011, 30 pp.; 2011, arXiv: 1104.4523.

[28] Р. Стонг, Заметки по теории кобордизмов, Мир, М., 1973, 372 с.; пер. с англ.: R. Stong, Notes on cobordism theory, Math. Notes, Univ. of Tokyo Press, Tokyo, 1968, v+354+lvi pp.

[29] F. Hirzebruch, "A Riemann-Roch theorem for differentiable manifolds", Séminaire N. Bourbaki, 1958/59, Exp. № 177, 1959, 129-149.

[30] M.F. Atiyah, F. Hirzebruch, "Riemann-Roch theorems for differentiable manifolds", Bull. Amer. Math. Soc., 65 (1959), 276-281.

[31] Э. Дайер, “Соотношения между теориями когомологий”, Математика, 9:2 (1965), 15-18; пер. с англ.: Е. Dyer, "Relations between cohomology theories", Colloquium on Algebraic Topology (Aarhus, 1962), Aarhus University, Aarhus, Denmark, 1962, 89-93.

[32] I. Panin, "Oriented cohomology theories of algebraic varieties", K-Theory, 30:3 (2003), 265-314.

[33] I. Panin, A. Smirnov, "Riemann-Roch theorems for oriented cohomology", $A x$ iomatic, enriched and motivic homotopy theory, NATO Sci. Ser. II Math. Phys. Chem., 131, Kluwer Acad. Publ., Dordrecht, 2004, 261-333.

[34] I. Panin, "Oriented cohomology theories of algebraic varieties. II. (After I. Panin and A. Smirnov.)", Homology, Homotopy Appl., 11:1 (2009), 349-405.

[35] D. Quillen, "Elementary proofs of some results of cobordism theory using Steenrod operations", Adv. in Math., 7 (1971), 29-56.

[36] V. Buchstaber, N. Ray, "Operations and quantum doubles in complex oriented cohomology theory", Homology, Homotopy Appl., 1 (1999), 169-185 (electronic).

[37] В. М. Бухштабер, С.П. Новиков, "Формальные группы, степенные системы и операторы Адамса”, Матем. сб., 84(126):1 (1971), 81-118; англ. пер.: V.M. Buhštaber, S.P. Novikov, "Formal groups, power systems and Adams operators", Math. USSR-Sb., 13:1 (1971), 80-116.

[38] В. М. Бухштабер, "Характер Чженя-Дольда в кобордизмах. I", Матем. сб., 83(125):4(12) (1970), 575-595; англ. пер.: V. M. Buhštaber, "The Chern-Dold character in cobordisms. I", Math. USSR-Sb., 12:4 (1970), 573-594.

[39] S. Ochanine, "Sur les genres multiplicatifs définis par des intégrales elliptiques", Topology, 26:2 (1987), 143-151.

[40] F. Hirzebruch, T. Berger, R. Jung, Manifolds and modular forms, Aspects Math., E20, Friedr. Vieweg \& Sohn, Braunschweig, 1992, xii+211 pp.

[41] D. Quillen, "On the formal group laws of unoriented and complex cobordism theory", Bull. Amer. Math. Soc., 75 (1969), 1293-1298.

[42] M. Lazard, "Sur les groupes de Lie formels à un paramètre", Bull. Soc. Math. France, 83 (1955), 251-274.

[43] E. Friedlander, "An introduction to K-theory", Some recent developments in algebraic K-theory, ICTP Lect. Notes, 23, Abdus Salam Int. Cent. Theoret. Phys., Trieste, 2008, 1-77.

[44] А. Дольд, "Соотношения между ординарными и экстраординарными теориями гомологий", Математика, 9:2 (1965), 8-14; пер. с англ.: A. Dold, "Relations between ordinary and extraordinary homology", Colloquium on Algebraic Topology (Aarhus, 1962), Aarhus University, Aarhus, Denmark, 1962, 2-9. 
[45] И.М. Кричевер, "Формальные группы и формула Атьи-Хирцебруха", Изв. АН СССР. Сер. матем., 38:6 (1974), 1289-1304; англ. пер.: I. M. Kričever, "Formal groups and the Atiyah-Hirzebruch formula", Math. USSR-Izv., 8:6 (1974), 1271-1285.

[46] И. М. Кричевер, “Обобщенные эллиптические роды и функции Бейкера-Ахиезера", Матем. заметки, 47:2 (1990), 34-45; англ. пер.: I. M. Krichever, "Generalized elliptic genera and Baker-Akhiezer functions", Math. Notes, 47:2 (1990), 132-142.

[47] J.F. Adams, "On Chern characters and the structure of the unitary group", Proc. Cambridge Philos. Soc., 57:2 (1961), 189-199.

[48] J.F. Adams, "On the groups $J(X)$. IV", Topology, 5 (1966), 21-71.

[49] И. М. Джеймс, "Приведенные степени пространств", Математика, 1:3 (1957), 3-34; пер. с англ.: I. M. James, "Reduced product spaces", Ann. of Math. (2), 62 (1955), 170-197.

[50] T. Coates, A. Givental, "Quantum Riemann-Roch, Lefschetz and Serre", Ann. of Math. (2), 165:1 (2007), 15-53.

[51] В. М. Бухштабер, "Модули дифференциалов спектральной последовательности Атья-Хирцебруха", Матем. сб., 78(120):2 (1969), 307-320; англ. пер.: V.M. Buhštaber, "Modules of differentials of the Atiyah-Hirzebruch spectral sequence", Math. USSR-Sb., 7:2 (1969), 299-313.

[52] M.F. Atiyah, F. Hirzebruch, "Analytic cycles on complex manifolds", Topology, 1:1 (1962), 25-45.

[53] B. Totaro, "Torsion algebraic cycles and complex cobordism", J. Amer. Math. Soc., 10:2 (1997), 467-493.

[54] Б. И. Ботвинник, В. М. Бухштабер, С. П. Новиков, С. А. Юзвинский, "Алгебраические аспекты теории умножений в комплексных кобордизмах", УМН, 55:4(334) (2000), 5-24; англ. пер.: B. I. Botvinnik, V. M. Buchstaber, S. P. Novikov, S. A. Yuzvinskii, "Algebraic aspects of the theory of multiplications in complex cobordism theory", Russian Math. Surveys, 55:4 (2000), 613-633.

[55] E. H. Brown, F.P. Peterson, "A spectrum whose $Z_{p}$ cohomology is the algebra of reduced $p^{\text {th }}$ powers", Topology, 5 (1966), 149-154.

[56] В. М. Бухштабер, "Проекторы в унитарных кобордизмах, связанные с SU-теорией", УМН, 27:6(168) (1972), 231-232.

[57] В. М. Бухштабер, “Двузначные формальные группы. Некоторые приложения к кобордизмам", УМН, 26:3(159) (1971), 195-196.

[58] В. М. Бухштабер, "Характеристические классы в кобордизмах и топологические приложения теорий однозначных и двузначных формальных групп”, Итоги науки и техники. Соврем. пробл. матем., 10, ВИНИТИ, М., 1978, 5-178; англ. пер.: V.M. Bukhshtaber, "Characteristic cobordism classes and topological applications of the theories of one-valued and two-valued formal groups", J. Soviet Math., 11:6 (1979), 815-921.

[59] V.L. Ginzburg, V. Guillemin, Y. Karshon, Moment maps, cobordisms, and Hamiltonian group actions, Math. Surveys Monogr., 98, Amer. Math. Soc., Providence, RI, 2002, viii+350 pp.

[60] В. В. Вершинин, “О кольце симплектических кобордизмов”, Топология, Сборник работ, Тр. МИАН СССР, 154, 1983, 49-52; англ. пер.: V. V. Vershinin, "The ring of symplectic cobordisms", Proc. Steklov Inst. Math., 154 (1985), 53-56.

[61] В. М. Бухштабер, А.Н. Холодов, "Топологические конструкции, связанные с многозначными формальными группами", Изв. АН СССР. Сер. матем., 46:1 (1982), 3-27; англ. пер.: V. M. Buchstaber, A. N. Kholodov, "Topological constructions connected with many-valued formal groups", Math. USSR-Izv., 20:1 (1983), 1-25. 
[62] V. M. Buchstaber, " $n$-valued groups: theory and applications", Mosc. Math. J., 6:1 (2006), 57-84.

[63] В. М. Бухштабер, А. В. Шокуров, “Алгебра Ландвебера-Новикова и формальные векторные поля на прямой”, Функи. анализ и его прил., 12:3 (1978), 1-11; англ. пер.: V. M. Bukhshtaber, A. V. Shokurov, "The Landweber-Novikov algebra and formal vector fields on the line", Funct. Anal. Appl., 12:3 (1978), 159-168.

[64] П. Коннер, Э. Флойд, Гладкие периодические отображения, Мир, М., 1969, 340 с.; пер. с англ.: P.E. Conner, E.E. Floyd, Differentiable periodic maps, Ergeb. Math. Grenzgeb., 33, Academic Press, New York; Springer-Verlag, Berlin-Göttingen-Heidelberg, 1964, vii+148 pp.

[65] А. С. Мищенко, "Многообразие с действием группы $Z_{p}$ и неподвижные точки", Матем. заметки, 4:4 (1968), 381-386; англ. пер.: А. S. Mishchenko, "Manifolds with the action of the group $Z_{p}$ and fixed points", Math. Notes, 4:4 (1968), 721-724.

[66] А. С. Мищенко, "Бордизмы с действием группы $Z_{p}$ и неподвижные точки", $M a$ тем. сб., 80(122):3(11) (1969), 307-313; англ. пер.: A. S. Mishchenko, "Bordisms with the action of the group $Z_{p}$ and fixed points", Math. USSR-Sb., 9:3 (1969), 291-296.

[67] Г.Г. Каспаров, "Инварианты классических линзовых многообразий в теории кобордизмов", Изв. АН СССР. Сер. матем., 33:4 (1969), 735-747; англ. пер.: G. G. Kasparov, "Invariants of classical lens manifolds in cobordism theory", Math. USSR-Izv., 3:4 (1969), 695-705.

[68] И. М. Кричевер, “Действия конечных циклических групп на квазикомплексных многообразиях", Матем. сб., 90(132):2 (1973), 306-319; англ. пер.: I. M. Krichever, "Actions of finite cyclic groups on quasicomplex manifolds", Math. USSR-Sb., 19:2 (1973), 305-319.

[69] С. М. Гусейн-Заде, И. М. Кричевер, "О формулах для неподвижных точек действия группы $Z_{p}$ ", УМH, 28:1(169) (1973), 237-238.

[70] И. М. Кричевер, “Замечание к работе 'Действия конечных циклических групп на квазикомплексных многообразиях'”, Матем. сб., 95(137):1(9) (1974), 146-147; англ. пер.: I. M. Krichever, "Remark on the paper 'Actions of finite cyclic groups on quasicomplex manifolds'", Math. USSR-Sb., 24:1 (1974), 145-146.

[71] С. М. Гусейн-Заде, "Неподвижные точки $U$-действий окружности", $У M H$, 26:4(160) (1971), 250.

[72] С. М. Гусейн-Заде, "О действии окружности на многообразиях", Матем. заметки, 10:5 (1971), 511-518; англ. пер.: S. M. Gusein-Zade, "On the action of a circle on manifolds", Math. Notes, 10:5 (1971), 731-734.

[73] С. М. Гусейн-Заде, "U-действия окружности и неподвижные точки", Изв. $A H$ СССР. Сер. матем., 35:5 (1971), 1120-1136; англ. пер.: S. M. Gusein-Zade, " $U$-actions of a circle and fixed points", Math. USSR-Izv., 5:5 (1971), 1127-1143.

[74] И. М. Кричевер, "Эквивариантные роды Хирцебруха. Формула Атья-Хирцебруха", УМН, 30:1(181) (1975), 243-244.

[75] Т.Е. Панов, "Вычисление родов Хирцебруха многообразий, несущих действие группы Z $/ p$, через инварианты действия", Известия РАН. Сер. матем., 62:3 (1998), 87-120; англ. пер.: Т. Е. Panov, "Calculation of Hirzebruch genera for manifolds acted on by the group $\mathbf{Z} / p$ via invariants of the action", Izv. Math., 62:3 (1998), 515-548.

[76] Ф. Хирцебрух, Топологические методы в алгебраической геометрии, Мир, M., 1973, 280 с.; пер. с нем.: F. Hirzebruch, Neue topologische Methoden in der algebraischen Geometrie, Ergeb. Math. Grenzgeb., 9, Springer-Verlag, Berlin-Göttingen-Heidelberg, 1956, viii+165 pp.

[77] В.М.Бухштабер, Н. Рэй, "Универсальный эквивариантный род и формула Кричевера", УМH, 62:1(373) (2007), 195-196; англ. пер.: V. M. Buchstaber, N. Ray, 
"Universal equivariant genus and Krichever's formula", Russian Math. Surveys, 62:1 (2007), 178-180.

[78] Т. Е. Панов, "Комбинаторные формулы для $\chi_{y}$-рода полиориентированного квазиторического многообразия", УМH, 54:5(329) (1999), 169-170; англ. пер.: T. E. Panov, "Combinatorial formulae for the $\chi_{y}$-genus of a multioriented quasitoric manifold", Russian Math. Surveys, 54:5 (1999), 1037-1039.

[79] Т. Е. Панов, "Роды Хирцебруха многообразий с действием тора", Изв. РАН. Сер. матем., 65:3 (2001), 123-138; англ. пер.: Т. E. Panov, "Hirzebruch genera of manifolds with torus action", Izv. Math., 65:3 (2001), 543-556.

[80] В. М. Бухштабер, Т. Е. Панов, Торические действия в топологии и комбинаторике, МЦНМО, М., 2004, 272 с.

[81] E. Thomas, "Complex structures on real vector bundles", Amer. J. Math., 89 (1967), 887-908.

[82] V. M. Buchstaber, T. E. Panov, N. Ray, "Toric genera", Int. Math. Res. Not., 16 (2010), 3207-3262.

[83] M. W. Davis, T. Januszkiewicz, "Convex polytopes, Coxeter orbifolds and torus actions", Duke Math. J., 62:2 (1991), 417-451.

[84] V.V. Batyrev, "Quantum cohomology rings of toric manifolds", Journées de géométrie algébrique d'Orsay (Orsay, 1992), Astérisque, 218, 1993, 9-34.

[85] В. М. Бухштабер, Н. Рай, “Торические многообразия и комплексные кобордизмы”, УМН, 53:2(320) (1998), 139-140; англ. пер.: V. M. Buchstaber, N. Ray, "Toric manifolds and complex cobordisms", Russian Math. Surveys, 53:2 (1998), 371-373.

[86] V.M. Buchstaber, T.E. Panov, N. Ray, "Spaces of polytopes and cobordism of quasitoric manifolds", Mosc. Math. J., 7:2 (2007), 219-242.

[87] А.А. Кустарев, “Эквивариантные почти комплексные структуры на квазиторических многообразиях", УМН, 64:1(385) (2009), 153-154; англ. пер.: A. A. Kustarev, "Equivariant almost complex structures on quasi-toric manifolds", Russian Math. Surveys, 64:1 (2009), 156-158.

[88] V.M. Buchstaber, S. Terzic, "Equivariant complex structures on homogeneous spaces and their cobordism classes", Geometry, topology, and mathematical physics, S. P. Novikov's seminar: 2006-2007, Amer. Math. Soc. Transl. Ser. 2, 224, eds. V. M. Buchstaber, I. M. Krichever, Amer. Math. Soc., Providence, RI, 2008, $27-57$.

[89] Т. Хонда, "Формальные группы и дзета-функции", Математика, 6 (1969), 3-17; пер. с англ.: Т. Honda, "Formal groups and zeta-functions", Osaka J. Math., 5 (1968), 199-213.

[90] M. Atiyah, F. Hirzebruch, "Spin-manifolds and group actions", Essays on topology and related topics, Mémoires dédiés à Georges de Rham, Springer, New York, 1970, $18-28$.

[91] B. Totaro, "Chern numbers for singular varieties and elliptic homology", Ann. of Math. (2), 151:2 (2000), 757-791.

[92] H. W. Braden, K.E. Feldman, "Functional equations and the generalised elliptic genus", J. Nonlinear Math. Phys., 12, Suppl. 1 (2005), 74-85.

[93] В. М. Бухштабер, Е. Ю. Бунькова, "Формальные группы Кричевера", Функи. анализ и его прил., 45:2 (2011), 23-44; англ. пер.: V. M. Buchstaber, E. Yu. Bun'kova, "Krichever formal groups", Funct. Anal. Appl., 45:2 (2011), 99-116.

[94] J. T. Tate, "The arithmetic of elliptic curves", Invent. Math., 23:3-4 (1974), 179-206.

[95] Э. Т. Уиттекер, Дж. Н. Ватсон, Курс современного анализа. ч. 2. Трансцендентные функиии, УРСC, М., 2010, 500 с.; пер. с англ.: Е. T. Whittaker, G. N. Watson, A course of modern analysis, Reprint of the fourth (1927) edition. An introduction to the general theory of infinite processes and of analytic functions; with an account 
of the principal transcendental functions, Cambridge Math. Lib., Cambridge Univ. Press, Cambridge, 1996, vi+608 pp.

[96] Ф. Хирцебрух, "Комплексные многообразия", Международный математический конгресс в Эдинбурге 1958 г., Физматгиз, М., 1962, 138-157; пер. с англ.: F. Hirzebruch, "Komplexe Mannigfaltigkeiten", Proc. Internat. Congress Math. 1958, Cambridge Univ. Press, New York, 1960, 119-136.

[97] R. E. Stong, "Relations among characteristic numbers. I", Topology, 4:3 (1965), 267-281; "Relations among characteristic numbers. II", Topology, 5:2 (1966), $133-148$.

[98] A. Hattori, "Integral characteristic numbers for weakly almost complex manifolds", Topology, 5:3 (1966), 259-280.

[99] С. Д. Ошанин, "Сигнатура SU-многообразий”, Матем. заметки, 13:1 (1973), 97-102; англ. пер.: S. D. Oshanin, "The signature of SU-varieties", Math. Notes, 13:1 (1973), 57-60.

[100] В. М. Бухштабер, А. П. Веселов, "Операторы Данкла, функциональные уравнения и преобразования эллиптических родов", УМН, 49:2(296) (1994), 147-148; англ. пер.: V. M. Buchstaber, A. P. Veselov, "Dunkl operators, functional equations, and transformations of elliptic genera", Russian Math. Surveys, 49:2 (1994), $145-147$.

[101] V. M. Buchstaber, A. P. Veselov, "On a remarkable functional equation in the theory of generalized Dunkl operators and transformations of elliptic genera", Math. Z., 223:4 (1996), 595-607.

[102] K. E. Feldman, "Chern numbers of Chern submanifolds", Q. J. Math., 53:4 (2002), 421-429.

[103] K. E. Feldman, "Miraculous cancellation and Pick's theorem", Toric topology, Contemp. Math., 460, Amer. Math. Soc., Providence, RI, 2008, 71-86.

[104] L. Alvarez-Gaumé, E. Witten, "Gravitational anomalies", Nucl. Phys. B, 234:2 (1984), 269-330.

[105] G. Pick, "Geometrisches zur Zahlenlehre", Sitzenber. Lotos Prag. (2), 19 (1900), 311-319.

[106] В. М. Бухштабер, "Группы полиномиальных преобразований прямой, неформальные симплектические многообразия и алгебра Ландвебера-Новикова", УМH, 54:4(328) (1999), 161-162; англ. пер.: V.M. Bukhshtaber, "Groups of polynomial transformations of a line, non-formal symplectic manifolds, and the Landweber-Novikov algebra", Russian Math. Surveys, 54:4 (1999), 837-838.

[107] И.К. Бабенко, И.А. Тайманов, "О неформальных односвязных симплектических многообразиях", Сиб. матем. журн., 41:2 (2000), 253-269; англ. пер.: I. K. Babenko, I. A. Taimanov, "On nonformal simply connected symplectic manifolds", Siberian Math. J., 41:2 (2000), 204-217.

[108] F. E. A. Johnson, E. G. Rees, "The fundamental groups of algebraic varieties", Algebraic topology Poznań 1989, Lecture Notes in Math., 1474, Springer, Berlin, 1991, $75-82$.

[109] И. К. Бабенко, С. А. Богатый, “Аменабельность группы подстановок формальных степенных рядов", Изв. РАН. Сер. матем., 75:2 (2011), 19-34; англ. пер.: I. K. Babenko, S. A. Bogatyi, "The amenability of the substitution group of formal power series", Izv. Math., 75:2 (2011), 239-252.

[110] И. К. Бабенко, С. А. Богатый, "О группе подстановок формальных целочисленных рядов", Изв. РАН. Сер. матем., 72:2 (2008), 39-64; англ. пер.: I. K. Babenko, S. A. Bogatyi, "On the group of substitutions of formal power series with integer coefficients", Izv. Math., 72:2 (2008), 241-264.

[111] И. К. Бабенко, “Алгебра, геометрия и топология группы подстановок формальных степенных рядов", УМН, 2013 (в печати). 
[112] I. M. Gel'fand, "The cohomology of infinite dimensional Lie algebra: some questions of integral geometry", Actes du Congrès International des Mathématiciens (Nice, 1970), 1, Gauthier-Villars, Paris, 1971, 95-111.

[113] Л.В.Гончарова, "Когомологии алгебр Ли формальных векторных полей на прямой”, Функи. анализ и его прил., 7:2 (1973), 6-14; англ. пер.: L. V. Goncharova, "The cohomologies of Lie algebras of formal vector fields on the line", Funct. Anal. Appl., 7:2 (1973), 91-97.

[114] Л.В.Гончарова, "Когомологии алгебр Ли формальных векторных полей на прямой”, Функи. анализ и его прил., 7:3 (1973), 33-44; англ. пер.: L. V. Goncharova, "The cohomologies of Lie algebras of formal vector fields on the line", Funct. Anal. Appl., 7:3 (1973), 194-203.

[115] Д. Б. Фукс, Когомологии бесконечномерных алгебр Ли, Наука, М., 1984, 272 с.; англ. пер.: D. B. Fuks, Cohomology of infinite-dimensional Lie algebras, Consultants Bureau, New York, 1986, xii+339 pp.

[116] А. И. Мальцев, "Об одном классе однородных пространств", Изв. АН СССР. Сер. матем., 13:1 (1949), 9-32; англ. пер.: А. I. Malcev, "On a class of homogeneous spaces", Amer. Math. Soc. Transl., 1951, № 39, 1951, 33 pp.

[117] Л. Ауслендер, Л. Грин, Ф. Хан, "Потоки на однородных пространствах", 1966, 208 с.; пер. с англ.: L. Auslander, L. Green, F. Hahn, "Flows on homogeneous spaces", Ann. of Math. Stud., 53, Princeton Univ. Press, Princeton, NJ, 1963, vii+107 pp.

[118] В. Снэйт, Алгебраический кобордизм и K-теория, Мир, М., 1983, 280 с.; пер. с англ.: V.P. Snaith, "Algebraic cobordism and K-theory", Mem. Amer. Math. Soc., 21:221 (1979), vii+152 pp.

[119] D. Gepner, V. Snaith, "On the motivic spectra representing algebraic cobordism and algebraic K-theory", Doc. Math., 14 (2009), 359-396.

[120] П. Коннер, Э. Флойд, “О соотношении теории кобордизмов и $K$-теории”, Дополнение к кн.: Гладкие периодические отображения, Мир, М., 1969, 233-331; пер. с англ.: P. E. Conner, E. E. Floyd, The relation of cobordism to K-theories, Lecture Notes in Math., 28, Springer-Verlag, Berlin-New York, 1966, v+112 pp.

[121] V. Voevodsky, The Milnor conjecture, 1996, http://www.math.uiuc.edu/K-theory/ 0170 .

[122] M. Levine, R. Pandharipande, "Algebraic cobordism revisited", Invent. Math., 176:1 (2009), 63-130.

[123] I. Panin, K. Pimenov, O. Roendigs, A universality theorem for Voevodsky's algebraic cobordism spectrum, 2007, http://www.math.uiuc.edu/K-theory/0846.

[124] A. Vishik, "Symmetric operations in algebraic cobordism", Adv. Math., 213:2 (2007), $489-552$. 